Preprint typeset in JHEP style - HYPER VERSION

TAUP-2828/06

hep-th/0605118

\title{
Non-Critical Pure Spinor Superstrings
}

\author{
Ido Adam ${ }^{1}$, Pietro Antonio Grassi ${ }^{2} 34$, Luca Mazzucato ${ }^{1}$, \\ Yaron $\mathrm{Oz}^{1}$ and Shimon Yankielowicz ${ }^{1}$, \\ ${ }^{1}$ Raymond and Beverly Sackler Faculty of Exact Sciences \\ School of Physics and Astronomy \\ Tel-Aviv University, Ramat-Aviv 69978, Israel \\ 2 Centro Studi e Ricerche E. Fermi, Compendio Viminale, I-00184, Roma, Italy \\ ${ }^{3}$ CERN, Theory Unit, CH-1211 Geneve, 23, Switzerland \\ 4 DISTA, Università del Piemonte Orientale, Via Bellini 25/G, 15100, \\ Alessandria, Italy and INFN, Torino, Italy \\ E-mails: adamido@post.tau.ac.il, pietro.grassi@cern.ch, \\ mazzul@post.tau.ac.il, yaronoz@post.tau.ac.il, \\ shimonya@post.tau.ac.il
}

\begin{abstract}
We construct non-critical pure spinor superstrings in two, four and six dimensions. We find explicitly the map between the RNS variables and the pure spinor ones in the linear dilaton background. The RNS variables map onto a patch of the pure spinor space and the holomorphic top form on the pure spinor space is an essential ingredient of the mapping. A basic feature of the map is the requirement of doubling the superspace, which we analyze in detail. We study the structure of the non-critical pure spinor space, which is different from the ten-dimensional one, and its quantum anomalies. We compute the pure spinor lowest lying BRST cohomology and find an agreement with the RNS spectra. The analysis is generalized to curved backgrounds and we construct as an example the non-critical pure spinor type IIA superstring on $A d S_{4}$ with RR 4-form flux.
\end{abstract}




\section{Contents}

1. Introduction and summary 2

2. The pure spinor formalism 7

2.1 The ten-dimensional map 11

3. RNS non-critical superstrings 14

3.1 Space-time supersymmetry

3.2 Spectrum 16

4. Two-dimensional superstrings 19

4.1 The RNS superstring

4.2 Multiplet spectra: RNS analysis 21

4.2.1 Holomorphic sector 21

4.2 .2 Closed superstring 22

4.3 Pure spinor variables 223

4.3.1 Supersymmetry structure

4.3.2 Cohomology 26

4.3.3 Curved non-critical backgrounds 29

4.3.4 Anomalies 30

5. Four-dimensional superstrings 31

5.1 Multiplet spectra: RNS analysis 31

5.1.1 Holomorphic sector 31

5.1.2 Closed superstring

5.2 Pure spinor variables 34

5.2.1 Supersymmetry structure 37

5.2 .2 Cohomology 39

5.2 .3 Curved non-critical backgrounds

5.2 .4 Anomalies 43

6. Six-dimensional superstrings 43

6.1 Multiplet spectra: RNS analysis 43

6.1 .1 Holomorphic sector 44

6.1.2 Closed superstring 45

6.2 Pure spinor variables 46

6.2 .1 Supersymmetry algebra 47

6.2 .2 Cohomology 48

6.2 .3 Curved non-critical backgrounds 50 
6.2.4 Anomalies 50

7. The pure spinor measure 51

8. Curved backgrounds 53

9. Discussion and open problems

A. Notations 57

B. Non-critical RNS superstrings 59

B.1 The matter system 59

B.2 The ghost system 60

B.3 Supersymmetry 61

B.3.1 The $N=2$ superconformal algebra 61

B.3.2 The twisted $N=2$ algebra 62

C. The RNS spectra 62

C.1 $\mathrm{d}=2$

C.1.1 Holomorphic sector 62

C.1.2 Closed superstring 63

C.2 $d=4$

C.2.1 Holomorphic sector 65

C.2.2 Closed superstring 66

D. Pure spinor cohomology 67

D.1 $d=0$ gauge supermultiplet 67

D.2 $d=2$ gauge supermultiplet 68

E. The six-dimensional map 70

F. A projection 72

\section{Introduction and summary}

The critical dimension for the superstrings in flat space-time is $d=10$. In dimensions $d<10$, the Liouville mode is dynamical and needs to be quantized as well. These superstrings are sometimes called non-critical. The Liouville mode can be interpreted as a dynamically generated dimension. Thus, if we start with superstring theory in $d<10$ space-time dimensions, we have effectively $d+1$ space-time dimensions. The 
total conformal anomaly vanishes for the non-critical superstrings due to the Liouville background charge. However, while this is a necessary condition for the consistency of non-critical superstrings, it is not a sufficient one. Much work has been done on the analysis of non-critical strings in two and less dimensions. Consistent superstring theories in linear dilaton backgrounds with even dimensions have been constructed in [1], and studied in the RNS formalism.

There are various motivations to study non-critical strings. First, non-critical superstrings can provide alternative to superstring compactifications. Second, the study of non-critical superstrings in the context of the gauge/string correspondence may provide dual descriptions of new gauge theories, and in particular QCD [2, 3].

A complication in the study of non-critical superstrings in curved spaces is that, unlike the critical case, there is no consistent approximation where supergravity provides a valid effective description. The reason being that the $d$-dimensional supergravity low-energy effective action contains a cosmological constant type term of the form

$$
S \sim \int d^{d} x \sqrt{G} e^{-2 \Phi}\left(\frac{d-10}{l_{s}^{2}}\right)
$$

which vanishes only for $d=10$. This implies that the low energy approximation $E \ll l_{s}^{-1}$ is not valid when $d \neq 10$, and the higher order curvature terms of the form $\left(l_{s}^{2} \mathcal{R}\right)^{n}$ cannot be discarded. A manifestation of this is that solutions of the $d$-dimensional non-critical supergravity equations have typically curvatures of the order of the string scale $l_{s}^{2} \mathcal{R} \sim O(1)$ when $d \neq 10$.

The second complication is that interesting target space curved geometries include RR field fluxes. As in the critical superstrings case, the RNS formulation is inadequate for the quantization of superstrings in such backgrounds. In [4] a covariant description of non-critical superstrings in even dimensions has been constructed using the hybrid type variables. The approach taken was to construct a covariant description of non-critical superstrings on the linear dilaton background and use the supersymmetric variables to construct the non-critical superstrings $\sigma$-model action in general curved target space backgrounds. The goal of this work is to develop the pure spinor quantization procedure for non-critical superstrings.

The pure spinor formalism for critical superstrings [5] is based on integer worldsheet spin variables. They are the target superspace coordinates $\left(x^{m}, \theta^{\alpha}\right)$, where $x^{m}, m=0, \ldots, 9$ are commuting coordinates and $\theta^{\alpha}, \alpha=1, \ldots, 16$ are anti-commuting coordinates, $p_{\alpha}$ the conjugate momenta to $\theta^{\alpha}$, the bosonic spinor ghosts $\lambda^{\alpha}$, which satisfy the (complex) pure spinor constraint

$$
\lambda^{\alpha} \gamma_{\alpha \beta}^{m} \lambda^{\beta}=0
$$

and their conjugate momenta $w_{\alpha}$. By construction, the pure spinor formalism is manifestly space-time supersymmetric and provides a simple coupling to the NS-NS and RR fields. 
Unlike the GS formalism, there is no $\kappa$-symmetry to gauge fix. Instead, an important ingredient of the pure spinor formalism is the nilpotent BRST operator

$$
Q=\int d z \lambda^{\alpha} d_{\alpha}
$$

where $d_{\alpha}$ acts as the supersymmetric derivative in ten dimensions. Physical states are elements in the BRST cohomology modulo the pure spinor constraints. In addition, the invariance of the action under the BRST symmetry can provide strong constraints on possible quantum corrections. This has been used, for instance, to prove that $A d S_{5} \times S^{5}$ is a consistent background for type II superstrings [6].

In this paper we will use a pure spinor formalism to describe non-critical superstrings. The strategy of constructing the pure spinor description of the non-critical superstrings is to first map the bosonic and fermionic linear dilaton RNS variables to pure spinor variables. A generic feature of the map in all dimensions is that it takes the RNS variables to the pure spinor variables on a patch of the pure spinor space. The pure spinor space of non-critical superstrings will be different from the pure spinor space of critical superstrings.

By performing the map of the RNS variables to the pure spinor variables, we will see that a basic feature is the requirement of doubling the superspace. This can be done by enlarging the linear dilaton superspace structure to include superspace coordinates (with their conjugate momenta), which are not BRST invariant on the RNS side. Similarly we will have a doubling of the superderivates, with only half of them being physical, though all being conserved. We will see that the current algebra of the doubled superderivatives is not a supersymmetry algebra and only a nonanomalous subsector closes on the spacetime supersymmetry. Indeed, working in a doubled superspace with the pure spinor variables will require an appropriate projection to this physical subsector. However, the doubled superspace will allow to study pure spinor superstrings in backgrounds with double the supersymmetries of the linear dilaton background.

Let us illustrate the above discussion by briefly presenting the pure spinor structure that we will find for two-dimensional superstrings in linear dilaton background

$$
\mathbb{R}_{\varphi} \times U(1)_{x}
$$

The background has two bosonic dimensions, the noncompact Liouville direction $\varphi$ and the $x$ direction compactified on a circle with radius $R=2 / Q$, where $Q=2$ is the Liouville background charge. The doubled superspace (in the holomorphic sector) has two fermionic coordinates $\left(\theta^{+}, \theta^{\dot{+}}\right)$ with their conjugate momenta $\left(p_{+}, p_{\dot{+}}\right)$. The $\left(\theta^{+}, p_{+}\right)$pair are BRST invariant physical quantities, while $\left(\theta^{\dot{+}}, p_{\dot{+}}\right)$ are BRST noninvariant, but required by the map from the RNS variables to the covariant variables. Similarly, the corresponding two superderivatives are $d_{+}$and $d_{\dot{+}}$, where the former is 
BRST invariant, and the latter is not. The OPE between the physical and unphysical $d$ 's does not close a superalgebra, but rather has a double pole

$$
d_{+}(z) d_{\dot{+}}(0) \sim-\frac{Q}{z^{2}}+\ldots
$$

The space-time zero-dimensional supersymmetry is realized by the physical $d_{+}$, which is in fact nilpotent in this case.

The crucial ingredient in this covariant formalism is provided by the pure spinor variables $\left(\lambda^{\alpha}, w_{\alpha}\right)$, which form a curved beta-gamma system on the pure spinor space. This has important consequences that will be discussed in detail in the paper. First there is a coupling of the worldsheet curvature $r^{(2)}$ to the holomorphic top form $\Omega$ on the pure spinor space

$$
S \sim \int d^{2} z r^{(2)} \log \Omega(\lambda),
$$

which modifies the stress tensor, as well as the saturation rules for correlators. Second, there are global obstructions to the definition of the pure spinor system on the worldsheet and on target space, associated with the need for holomorphic transition functions relating $\left(\lambda^{\alpha}, w_{\alpha}\right)$ on different patches of the pure spinor space, which are compatible with their OPE. They are reflected by quantum anomalies in the worldsheet and pure spinor space holomorphic diffeomorphisms [0]. The critical superstring pure spinor space has a singularity at $\lambda^{\alpha}=0$. Blowing up the singularity results in an anomalous theory. However, simply removing the origin leaves a nonanomalous theory [8]. The same holds for the pure spinor spaces of non-critical superstrings. However, in two and four dimensions, removing the origin results in disconnected pure spinor spaces.

The pure spinor variables in the two dimensional non-critical string are $\left(\lambda^{+}, \lambda^{+}\right)$ satisfying the equation

$$
\lambda^{+} \lambda^{\dot{+}}=0 .
$$

This defines a complex dimension one pure spinor space. Note for comparison that the pure spinor space of critical superstrings in ten dimensions is a complex elevendimensional space. The map from the RNS variables to the pure spinor ones takes the RNS variables to the patch of the pure spinor space defined by $\left(\lambda^{+} \neq 0, \lambda^{+}=0\right)$.

The superstring on a two dimensional linear dilaton background is defined by the following stress tensor

$$
\begin{gathered}
T=-\frac{1}{2} \Pi^{m} \Pi_{m}-d_{I} \partial \theta^{I}+\frac{Q}{2} \epsilon_{I J} \partial \theta^{I} \partial \theta^{J}+\frac{Q}{2} \partial^{2}\left(\Pi^{\varphi}-i \Pi^{x}\right) \\
+w_{I} \partial \lambda^{I}-\frac{1}{2} \partial^{2} \log \Omega(\lambda),
\end{gathered}
$$

where $I=+, \dot{+}$ and $m=x, \varphi$. This structure is very similar to that of pure spinor critical superstrings and is convenient for generalization to curved backgrounds. Note that the terms proportional to $Q=2$ are features of the linear dilaton background. 
In the BRST operator of the non-critical superstrings we will include not only the physical superderivatives but also the unphysical ones. Note in comparison that, in the critical superstring in flat ten dimensions, all the superderivatives in the BRST charge are physical. Here, the BRST operator

$$
Q_{B}=\oint \lambda^{I} d_{I}
$$

where $I=+, \dot{+}$, includes both the physical and unphysical superderivatives. Note that, due to the double pole in the $d^{\prime}$ 's OPE, the nilpotency of the BRST charge $Q_{B}$ requires, in addition to the pure spinor condition $\lambda^{+} \lambda^{\dot{+}}=0$, that

$$
\partial \lambda^{+} \lambda^{\dot{+}}-\lambda^{+} \partial \lambda^{\dot{+}}=0
$$

However, this derivative condition is a consequence of the algebraic one.

We will compute the cohomology and, since we are interested in the space-time supersymmetry multiplets, we will consider only the part of the cohomology that contains the variables that realize the supersymmetry current algebra. The physical states are the vertex operators at ghost number one and weight zero. We will find two different kinds of such operators. The first is analogous to the usual ten-dimensional vertex operator. In general this contains the off-shell $d$-dimensional supergravity multiplet (for the closed strings). The second type is peculiar to the linear dilaton background. It contains a gauge multiplet, in which the tachyon sits. This will reproduce the RNS computation of the short supermultiplets.

In computing the cohomology of the BRST operator, we restrict to the part of the vertex operators that contains the physical fermionic coordinate $\theta^{+}$. This indeed reproduces the RNS computation of the short supermultiplets. The supergravity multiplet does not exist in the case of two-dimensional superstrings, while the supermultiplet in which the tachyon sits has two bosonic and two fermionic degrees of freedom.

One essential feature of the pure spinor non-critical string is that it can be generalized to describe other non-critical backgrounds, as we are used to do in the ten-dimensional critical superstrings. As an example, we will propose a sigma model for the type IIA non-critical superstring on $A d S_{4}$ background with $\mathrm{RR}$ four-form flux. This background is described by the supercoset

$$
\frac{O S p(2 \mid 4)}{S O(1,3) \times S O(2)}
$$

and has eight real supercharges, which is the content of the enlarged superspace in the four-dimensional non-critical string. As we will see, the action for this supercoset will be suitable for quantization and its BRST charge will be the usual pure spinor BRST charge. 
The paper is organized as follows. In section 2 we first give a brief introduction to the pure spinor formalism for critical ten-dimensional superstrings. We then construct a map from the RNS variables to the pure spinor ones for critical tendimensional superstrings. The map makes the $\beta \gamma$-system structure of the pure spinor variables explicit and we will gain an insight into the global definition of the pure spinor space and the importance of its holomorphic top form. In section 3 we discuss the RNS non-critical superstrings in the linear dilaton background and provide all the necessary ingredients for the rest of the paper. In particular we summarize the space-time supersymmetry structure and describe the spectrum of space-time supermultiplets.

In section 4 we pass to the construction of the pure spinor two-dimensional noncritical superstring, whose main features have been outlined above. We map the RNS variables to the pure spinor ones, analyze the supersymmetry structure of the covariant formulation and its pure spinor space. We construct the pure spinor action and stress tensor, introduce the BRST operator and its cohomology and consider global issues and anomalies of the pure spinor formalism. In section 5 and 6 we repeat the same construction and analysis for the pure spinor non-critical superstrings in four and in six dimensions.

In section 7 we comment on the computation of amplitudes and discuss the non-critical pure spinor measure. In section 8 we propose the type IIA pure spinor formulation of non-critical $A d S_{4}$ background with RR flux. Section 9 is devoted to a discussion of the open problems and the future directions. In the appendices we will put the details of various computations. In appendix A we explain the various notations for the spinors we used through the main text. In appendix $\mathrm{B}$ and $\mathrm{C}$ we collect the RNS generators of the $N=2$ superconformal algebra and we show some details of the RNS computation of the short multiplets. In appendix D and E we give some details of the pure spinor computations. In appendix $\mathrm{F}$ we present a curious deformation of the pure spinor theory we came accross.

\section{The pure spinor formalism}

In this section we will briefly review the main ingredients of the pure spinor formalism for critical superstrings in flat ten-dimensional target space [5, 9]. These structures will appear with some modifications in the pure spinor non-critical superstrings. We will consider for simplicity the open superstring. The generalization to the closed string case is straightforward. Consider the supermanifold $\left(x^{m}, \theta^{\alpha}\right)$, where $x^{m}, m=0, \ldots, 9$ are commuting coordinates and $\theta^{\alpha}, \alpha=1, \ldots, 16$ are anti-commuting coordinates. One introduces $p_{\alpha}$ as the conjugate momenta to $\theta^{\alpha}$ with the OPE

$$
p_{\alpha}(z) \theta^{\beta}(0) \sim \frac{\delta_{\alpha}^{\beta}}{z}
$$


Next we add bosonic spinor ghosts $\lambda^{\alpha}$, which satisfy the (complex) pure spinor constraint

$$
\lambda^{\alpha} \gamma_{\alpha \beta}^{m} \lambda^{\beta}=0,
$$

and their conjugate momenta $w_{\alpha}$. The system $\left(w_{\alpha}, \lambda^{\alpha}\right)$ is a $(\beta, \gamma)$ system of weights $(1,0) . \gamma_{\alpha \beta}^{m}$ are the symmetric $16 \times 16$ Pauli matrices in ten dimensions. The pure spinor set of constraints (2.2) is reducible. It defines a complex eleven-dimensional space $\mathcal{M}$, which is a cone over $\mathcal{Q}=\frac{S O(10)}{U(5)}$.

The pure spinor constraint implies that $w_{\alpha}$ are defined up to the gauge transformation

$$
\delta w_{\alpha}=\Lambda^{m}\left(\gamma_{m} \lambda\right)_{\alpha} .
$$

Therefore, $w_{\alpha}$ appears only in gauge invariant combinations. These are the Lorentz algebra currents $M_{m n}$, the ghost number current $J_{(w, \lambda)}$ which assigns ghost number 1 to $\lambda$ and ghost number -1 to $w$

$$
M_{m n}=\frac{1}{2} w \gamma_{m n} \lambda, \quad J_{(w, \lambda)}=w_{\alpha} \lambda^{\alpha},
$$

and the pure spinor stress-energy tensor $T_{(w, \lambda)}$, which we will now discuss.

The gauge fixed worldsheet action is $S=S_{0}+S_{1}$, where

$$
S_{0}=\int d^{2} z\left(\frac{1}{2} \partial x^{m} \bar{\partial} x_{m}+p_{\alpha} \bar{\partial} \theta^{\alpha}-w_{\alpha} \bar{\partial} \lambda^{\alpha}\right),
$$

and

$$
S_{1}=\int d^{2} z\left(\frac{1}{4} r^{(2)} \log \Omega(\lambda)\right) .
$$

$S_{1}$ is a coupling of the worldsheet curvature $r^{(2)}$ to the holomorphic top form $\Omega$ of the pure spinor space $\mathcal{M}$

$$
\Omega=\Omega(\lambda) d \lambda^{1} \wedge \ldots \wedge d \lambda^{11} .
$$

It is not yet clear, however, what is the non gauge-fixed form of the action $S .{ }^{1}$ Note that the $\left(w_{\alpha}, \lambda^{\alpha}\right)$ action is holomorphic and does not depend on their complex conjugates.

The stress tensor of the $(w, \lambda)$ system reads

$$
T_{(w, \lambda)}=w_{\alpha} \partial \lambda^{\alpha}-\frac{1}{2} \partial^{2} \log \Omega(\lambda),
$$

and we will discuss the significance of the last term below. The system $\left(w_{\alpha}, \lambda^{\alpha}\right)$ is interacting due to the pure spinor constraint. It has the central charge $c_{(w, \lambda)}=22$, which is twice the complex dimension of the pure spinor space

$$
T_{(w, \lambda)}(z) T_{(w, \lambda)}(0) \sim \frac{\operatorname{dim}_{\mathbb{C}}(\mathcal{M})}{z^{4}}+\ldots .
$$

\footnotetext{
${ }^{1}$ There have been various attempts to derive the pure spinor formalism from first principles [10, 11, 12].
} 
The ghost number anomaly reads

$$
J_{(w, \lambda)}(z) T_{(w, \lambda)}(0) \sim-\frac{8}{z^{3}}+\ldots=\frac{c_{1}(\mathcal{Q})}{z^{3}}+\ldots,
$$

where $c_{1}(\mathcal{Q})$ is the first Chern class of the pure spinor cone base $\mathcal{Q}$.

The physical states are defined as the ghost number one cohomology of the nilpotent BRST operator

$$
Q=\oint d z \lambda^{\alpha} d_{\alpha}
$$

where

$$
d_{\alpha}=p_{\alpha}-\frac{1}{2} \gamma_{\alpha \beta}^{m} \theta^{\beta} \partial x_{m}-\frac{1}{8} \gamma_{\alpha \beta}^{m} \gamma_{m \gamma \delta} \theta^{\beta} \theta^{\gamma} \partial \theta^{\delta} .
$$

This BRST operator is an essential ingredient of the formalism but it is not clear how to derive its form by a gauge fixing procedure.

The $d_{\alpha}$ are the supersymmetric Green-Schwarz constraints. They are holomorphic and satisfy the OPE

$$
d_{\alpha}(z) d_{\beta}(0) \sim-\frac{\gamma_{\alpha \beta}^{m} \Pi_{m}(0)}{z},
$$

and

$$
d_{\alpha}(z) \Pi^{m}(0) \sim \frac{\gamma_{\alpha \beta}^{m} \partial \theta^{\beta}(0)}{z}
$$

where

$$
\Pi_{m}=\partial x_{m}+\frac{1}{2} \theta \gamma_{m} \partial \theta,
$$

is the supersymmetric momentum. $d_{\alpha}$ acts on function on superspace $F\left(x^{m}, \theta^{\alpha}\right)$ as

$$
d_{\alpha}(z) F\left(x^{m}(0), \theta^{\beta}(0)\right) \sim \frac{D_{\alpha} F\left(x^{m}(0), \theta^{\beta}(0)\right)}{z},
$$

where

$$
D_{\alpha}=\frac{\partial}{\partial \theta^{\alpha}}+\frac{1}{2} \gamma_{\alpha \beta}^{m} \theta^{\beta} \partial_{m},
$$

is the supersymmetric derivative in ten dimensions.

Massless states are described by the ghost number one weight zero vertex operators

$$
\mathcal{V}^{(1)}=\lambda^{\alpha} A_{\alpha}(x, \theta) .
$$

The conditions $Q \mathcal{V}^{(1)}=0$ and the gauge invariance $\delta \mathcal{V}^{(1)}=Q \Omega^{(0)}$ imply by explicit computation that $A_{\alpha}$ is a super Maxwell spinor superfield in ten dimensions

$$
A_{\alpha}(x, \theta)=\frac{1}{2}\left(\gamma^{m} \theta\right)_{\alpha} a_{m}(x)+\frac{i}{12}\left(\theta \gamma^{m n p} \theta\right)\left(\gamma_{m n p}\right)_{\alpha \beta} \psi^{\beta}(x)+O\left(\theta^{3}\right)
$$

where $a_{m}(x)$ is the gauge field and $\psi^{\gamma}(x)$ is the gluino. It is related to the gauge field $A_{m}$ by

$$
A_{m}=\gamma_{m}^{\alpha \beta} D_{\alpha} A_{\beta}
$$


and $A_{m}(x, \theta)=a_{m}(x)+O(\theta)$. Only in ten dimensions do these conditions give an on-shell vector multiplet. In lower dimensions they describe an off-shell vector multiplet.

The integrated ghost number zero vertex operator for the massless states reads

$$
\mathcal{V}=\int d z\left(\partial \theta^{\alpha} A_{\alpha}+\Pi^{m} A_{m}+d_{\alpha} W^{\alpha}+\frac{1}{2} M_{m n} F^{m n}\right),
$$

where $W^{\alpha}$ and $F^{m n}$ are the spinorial and bosonic field strength, respectively. The analysis of the massive states proceeds in a similar way. At the first massive level the ghost number one weight one vertex operator has the expansion [13]

$$
\mathcal{U}^{(1)}=\partial \lambda^{\alpha} A_{\alpha}+\lambda^{\alpha} \partial \theta^{\beta} B_{\alpha \beta}+\ldots .
$$

In curved spaces the superspace field equations are derived by the requirement that $\lambda^{\alpha} d_{\alpha}$ is holomorphic and nilpotent [14].

The construction of the closed superstrings is straightforward. One introduces the right moving superspace variables $\left(\bar{p}_{\hat{\alpha}}, \bar{\theta}^{\hat{\alpha}}\right)$, the pure spinor system $\left(\bar{w}_{\hat{\alpha}}, \bar{\lambda}^{\hat{\alpha}}\right)$ and the nilpotent BRST operator

$$
\bar{Q}=\oint d \bar{z} \bar{\lambda}^{\hat{\alpha}} \bar{d}_{\hat{\alpha}}
$$

The analysis of the spectrum proceeds by combining the left and right sectors. For instance, the integrated ghost number zero vertex operator for the massless states reads

$$
\mathcal{U}=\int d^{2} z\left(\partial \theta^{\alpha} A_{\alpha \hat{\beta}} \bar{\partial} \bar{\theta}^{\hat{\beta}}+\partial \theta^{\alpha} A_{\alpha m} \bar{\Pi}^{m}+\ldots\right) .
$$

The pure spinor system $\left(\lambda^{\alpha}, w_{\alpha}\right)$ defines a non-linear $\sigma$-model due to the curved nature of the pure spinor space (2.2). There are global obstructions to define the pure spinor system on the worldsheet and on target space [7, 8]. They are associated with the need for holomorphic transition functions relating $\left(\lambda^{\alpha}, w_{\alpha}\right)$ on different patches of the pure spinor space, which are compatible with their OPE. They are reflected by quantum anomalies in the worldsheet and target space (pure spinor space) diffeomorphisms. The conditions for the vanishing of these anomalies are the vanishing of the integral characteristic classes

$$
\frac{1}{2} c_{1}(\Sigma) c_{1}(\mathcal{M})=0, \quad \frac{1}{2} p_{1}(\mathcal{M})=0,
$$

$c_{1}(\Sigma)$ is the first Chern class of the worldsheet Riemann surface, $c_{1}(\mathcal{M})$ is the first Chern class of the pure spinor space $\mathcal{M}$, and $p_{1}$ is the first Pontryagin class of the pure spinor space. The vanishing of $c_{1}(\mathcal{M})$ is needed for the definition of superstring perturbation theory and it implies the existence of the nowhere vanishing holomorphic top form $\Omega(\lambda)$ on the pure spinor space $\mathcal{M}$, that appears in the stress tensor (2.8). 
The pure spinor space (2.2) has a singularity at $\lambda^{\alpha}=0$. Blowing up the singularity results in an anomalous theory. However, simply removing the origin leaves a non-anomalous theory. This means that one should consider the pure spinor variables as twistor-like variables. Indeed this is a natural intrepretation of the pure spinor variables considering them from the twistor string point of view.

Finally, although we will not discuss the computation of loop amplitudes, it is worth mentioning that unlike the RNS superstrings, all the variables that we use in the pure spinor superstring are of integer worldsheet spin and there is no need to sum over spin structures.

\subsection{The ten-dimensional map}

In this section we will construct a map from the RNS variables to the pure spinor ones. We will make use of a parameterization of the pure spinor components that would make the $\beta \gamma$-system structure of the pure spinor variables explicit. In this way we will gain a new insight into the global definition of the pure spinor space and the importance of its holomorphic top form. The pure spinor stress tensor we will obtain by the map will contain the contribution of the holomorphic top form on the pure spinor space. Indeed this term is necessary for a consistent definition of the pure spinor $\beta \gamma$-system [8, [7. Note that in [15] a similar map from the RNS variables to the pure spinor ones has been constructed, but with no consideration of the $\beta \gamma$-system structure and the holomorphic top form.

In the following we will consider the holomorphic sector. It is to be complemented by the anti-holomorphic sector for obtaining the Type II superstring. The holomorphic supercharges in the $-\frac{1}{2}$ picture of the RNS superstring are given by the spin fields

$$
q_{\boldsymbol{s}}=e^{-\phi / 2+\sum_{I=1}^{5} s_{I} H^{I}}
$$

where the $H^{I}$ s are the bosons obtained from the bosonization of the RNS worldsheet matter fermions and the $s_{I}$ 's take the values $\pm \frac{1}{2}$. These supercharges decompose into two Weyl representations.

In order to proceed with the map, one must first solve the pure spinor constraint $\lambda \gamma^{m} \lambda=0$, going to one patch of this manifold. In each patch a different component of the pure spinor is non-zero. The field redefinition we will use maps the RNS description into one patch of the pure spinor manifold. For concreteness we will work on one of the patches which is most conveniently described by the $S U(5) \times U(1)$ decomposition of the pure spinor $\lambda^{\alpha}=\left(\lambda^{+}, \lambda^{a}, \lambda_{a b}\right)$. The component of the pure spinor assumed to be non-zero is $\lambda^{+}$corresponding to the representation $1_{\frac{5}{2}}$ of this decomposition. In this patch one can solve for the $5_{-\frac{3}{2}}$ components $\lambda^{a}$ in terms of $\lambda^{+}$and the components in the $10_{\frac{1}{2}}$ representation $\lambda_{a b}$. 
On this patch the supercharge $q_{+}$which is the singlet of $S U(5)$ is raised to the $+\frac{1}{2}$ picture:

$$
q_{+}=b \eta e^{3 \phi / 2+i \sum_{a} H^{a} / 2}+i \sum_{a} \partial\left(x^{a}+i x^{a+5}\right) e^{\phi / 2+i \sum_{b} H^{b} / 2-i H^{a}}
$$

while the supercharges $q_{a}$, corresponding to the pure spinor components $\lambda^{a}$ we solved for, remain in the $-\frac{1}{2}$ picture. Together they form a part of the original ten-dimensional supersymmetry algebra. One then defines the fermionic momenta

$$
p_{+}=b \eta e^{3 \phi / 2+i \sum H^{a} / 2}, \quad p_{a}=q_{a}
$$

and their conjugate coordinates $\theta^{+}$and $\theta^{a}$. Note that the OPE's of the fermionic momenta among themselves are all non-singular.

The heart of the map is the introduction of two new fields $\tilde{\phi}$ and $\tilde{\kappa}$ using

$$
\eta=e^{\tilde{\phi}+\tilde{\kappa}} p_{+}, \quad b=e^{(\tilde{\phi}-\tilde{\kappa}) / 2} p_{+},
$$

yielding

$$
\begin{aligned}
& \tilde{\phi}=-\frac{3 i}{4} \sum_{a} H^{a}-\kappa-\frac{9}{4} \phi+\frac{1}{2} \chi, \\
& \tilde{\kappa}=\frac{i}{4} \sum_{a} H^{a}-\kappa-\frac{3}{4} \phi-\frac{1}{2} \chi,
\end{aligned}
$$

whose OPE's are

$$
\tilde{\phi}(z) \tilde{\phi}(0) \sim-\log z, \quad \tilde{\kappa}(z) \tilde{\kappa}(0) \sim \log z .
$$

The reason why we choose the particular field redefinition (2.29), explained in [15], is that the pure spinor formalism is, loosely speaking, equivalent to the RNS formalism when we take into account all the different pictures at the same time, which is achieved by working in the large Hilbert space, that is including the zero modes of the ghost $\xi$. But the usual cohomology of the RNS BRST charge $Q_{R N S}$ in the small Hilbert space is equivalent to the cohomology of $Q_{R N S}+\oint \eta$ in the large Hilbert space. With the redefinition (2.29), we are then mapping the $\oint \eta$ term of this extended BRST charge directly to the part $\oint \lambda^{+} d_{+}$of the Berkovits BRST operator (2.11).

By substituting the map into the RNS energy-momentum tensor one obtains

$$
\begin{aligned}
T= & T_{\mathrm{m}}+T_{\mathrm{gh}}=-\frac{1}{2} \sum_{m}\left(\partial x^{m}\right)^{2}-p_{+} \partial \theta^{+}-\sum_{a} p_{a} \theta^{a}- \\
& -\frac{1}{2}(\partial \tilde{\phi})^{2}+\frac{1}{2}(\partial \tilde{\kappa})^{2}+\partial^{2} \tilde{\phi}+\partial^{2} \tilde{\kappa}
\end{aligned}
$$

This can be verified to have still a vanishing central charge

$$
c=(10)_{x}+(-12)_{p \theta}+(2)_{\tilde{\phi} \tilde{\kappa}}=0 .
$$


The pure spinors are reconstructed by the ordinary bosonization of a $\beta \gamma$-system [16]

$$
\lambda^{+}=e^{\tilde{\phi}+\tilde{\kappa}}, \quad w_{+}=\partial \tilde{\kappa} e^{-\tilde{\phi}-\tilde{\kappa}},
$$

whose OPE is

$$
w_{+}(z) \lambda^{+}(0) \sim \frac{1}{z} .
$$

But the naive stress tensor one would expect for this $\beta \gamma$-system

$$
w_{+} \partial \lambda^{+}=-\frac{1}{2}(\partial \tilde{\phi})^{2}+\frac{1}{2}(\partial \tilde{\kappa})^{2}-\frac{1}{2} \partial^{2} \tilde{\phi}-\frac{1}{2} \partial^{2} \tilde{\kappa}
$$

does not coincide with the one we got from the map (2.33). This shows that the pure spinor stress tensor is not simply $w_{+} \partial \lambda^{+}$but actually

$$
T_{\lambda}=w_{+} \partial \lambda^{+}-\frac{1}{2} \partial^{2} \log \Omega(\lambda)
$$

where $\Omega$ is the coefficient of a top form defined on the pure spinor space [8]. By comparison we can read off the top form itself

$$
\Omega=e^{-3(\tilde{\phi}+\tilde{\kappa})}=\left(\lambda^{+}\right)^{-3}
$$

At this point, we can map the RNS saturation rule for amplitudes on the sphere

$$
\left\langle c \partial c \partial^{2} c e^{-2 \phi}\right\rangle=1
$$

to the pure spinor variables, obtaining

$$
\left\langle\left(\lambda^{+}\right)^{3}\left(\theta^{a}\right)^{5}\right\rangle=1
$$

which is the prescription for the saturation of the zero modes in the Berkovits formalism [5]. Note that the third power of the pure spinor is consistent with the expression of the holomorphic top form (2.37) we just reconstructed.

The next step in performing the map is that we have to covariantize this superstring by adding the missing coordinates and momenta. So, following [15, we add a BRST quartet consisting of ten $(1,0) b c$-systems $\left(p_{a b}, \theta^{a b}\right)$ and ten $(1,0) \beta \gamma$-systems $\left(w_{a b}, \lambda^{a b}\right)$. They have opposite central charges, so the total central charge remains unchanged. In this way we recover the full pure spinor stress tensor

$$
T=-\frac{1}{2} \partial x^{m} \partial x_{m}-d_{\alpha} \partial \theta^{\alpha}+w_{\alpha} \partial \lambda^{\alpha}-\frac{1}{2} \partial^{2} \log \Omega(\lambda)
$$

The BRST charge has to be modified to assure that these extra degrees of freedom are not included in physical states. Since we will be concerned with non-critical supertrings in this paper, we leave for a future work the study of how the BRST cohomology of the RNS superstring is mapped to the pure spinor cohomology. 
Let us mention that the study of the global properties of the pure spinor space $\mathcal{M}$ is crucial in order to obtain the correct cohomology of the superstring. The curved space $\mathcal{M}$ can be covered by sixteen patches, one for each component of the pure spinor $\lambda^{\alpha}$ that we can take to be nonzero. On a single patch, the pure spinor action reduces to the sum of eleven free $\beta \gamma$-systems. If we want to recover the spectrum of the superstring from the cohomology of the free $\beta \gamma$-system, we need to add to the Berkovits operator (2.11) the Cech operator $\delta_{\text {Cech }}$ on the pure spinor space $\mathcal{M}$, such that the total BRST charge of the pure spinor $\beta \gamma$-system is actually

$$
Q_{B R S T}=\oint \lambda^{\alpha} d_{\alpha}-\delta_{C e c h}
$$

which computes the Cech cohomology on $\mathcal{M}$ with values in the BRST cohomology of (2.11). This will be discussed in a separate work.

\section{RNS non-critical superstrings}

In this section we will consider the RNS description of superstrings propagating in the $d+2$ dimensional background [1, 17]

$$
\mathbb{R}^{1, d-1} \times \mathbb{R}_{\varphi} \times U(1)_{x}
$$

with flat metric in the string frame and a linear dilaton

$$
\Phi=\frac{Q}{2} \varphi
$$

The effective string coupling $g_{s}=e^{\Phi}$ varies as we move along the $\varphi$ direction and when considering scattering processes one needs to properly regularize the region in which the coupling diverges. We will only consider the weak coupling region $\varphi=-\infty$, where perturbative string computations are valid and we can safely analyze the string spectrum.

In the following we will focus on the holomorphic sector of the closed superstring. The $d+2$ dimensional RNS superstring is described in the superconformal gauge by $2 n+1$ superfields $X^{\mu}$, with $\mu=1, \ldots, d=2 n$, and $X$ and by a Liouville superfield $\Phi_{l}$. In components we have $X^{\mu}=\left(x^{\mu}, \psi^{\mu}\right), X=\left(x, \psi_{x}\right)$ and $\Phi_{l}=\left(\varphi, \psi_{l}\right)$, where the $\psi$ 's are Majorana-Weyl fermions.

The $d=2 n$ coordinates $x^{\mu}$ parameterize the even dimensional flat Minkowski part of the space, while the coordinate $x$ is compactified on a circle of radius $R=2 / Q$, whose precise value is dictated by the requirement of space-time supersymmetry, as we will see below. The coordinate $\varphi$ parameterizes the linear dilaton direction with a background charge $Q$. As usual, we need to add the superdiffeomorphisms ghosts $(\beta, \gamma)$ and $(b, c)$. The central charge of the system is

$$
c=(3 / 2)(2 n+1)_{\left\{X^{\mu}, X\right\}}+\left(3 / 2+3 Q^{2}\right)_{\left\{\Phi_{l}\right\}}+(11)_{\{\beta \gamma\}}-(26)_{\{b c\}}
$$


and the requirement that it vanishes fixes the slope of the dilaton to $Q(n)=\sqrt{4-n}$. For $n=4$, the background charge vanishes and we have eight flat coordinates plus $\varphi$ and $x$, getting back to the flat ten-dimensional critical superstring. When $n \neq 4$ we have non-critical superstrings.

\subsection{Space-time supersymmetry}

In even dimensions $d=2 n$, at the particular value of the radius $R=2 / Q$ the worldsheet theory has a global $N=2$ superconformal symmetry. Before showing its generators, let us define $\Psi=\psi_{l}+i \psi_{x}$ and $\Psi^{I}=\psi^{I}+i \psi^{I+n}$ (with $I=1, \ldots, n$ ) and bosonize them in the usual way by introducing the bosonic fields $H, H^{I}$ and setting

$$
\Psi \Psi^{\dagger}=2 i \partial H, \quad \Psi^{I} \Psi^{I \dagger}=2 i \partial H^{I}
$$

where $\dagger$ denotes Hermitian conjugation in field space and the $H^{\prime}$ 's have canonical OPE's $H^{I}(z) H^{J}(w) \sim-\delta^{I J} \log (z-w)$. In this way we can define the spin fields $\Sigma^{ \pm}=e^{ \pm \frac{i}{2} H}$ in the $(x, \varphi)$ direction and the spin fields $\Sigma^{a}=e^{ \pm \frac{i}{2} H^{1} \ldots \pm \frac{i}{2} H^{n}}$, where the index $a$ runs over the independent spinor representation of $S O(2 n)$. We list below the matter part of the $\mathcal{N}=2$ superconformal generators, their ghost part is collected in appendix $\mathrm{A}$.

The matter stress tensor is

$$
T_{m}=-\frac{1}{2} \sum_{\mu=1}^{2 n}\left(\partial x^{\mu}\right)^{2}-\frac{1}{2} \sum_{I=1}^{n}\left(\partial H^{I}\right)^{2}-\frac{1}{2}(\partial x)^{2}-\frac{1}{2}(\partial \varphi)^{2}+\frac{Q}{2} \partial^{2} \varphi-\frac{1}{2}(\partial H)^{2},
$$

the two supercurrents are

$$
\begin{aligned}
& G^{+}=i \sum_{I=1}^{n} e^{-i H^{I}} \partial\left(x^{I}+i x^{I+n}\right)+i e^{-i H} \partial(\varphi+i x+i Q H), \\
& G^{-}=i \sum_{I=1}^{n} e^{i H^{I}} \partial\left(x^{I}-i x^{I+n}\right)+i e^{i H} \partial(\varphi-i x-i Q H),
\end{aligned}
$$

and the $U(1)$ current is

$$
J=-i \sum_{I=1}^{n} \partial H^{I}-i \partial H+i Q \partial x
$$

The worldsheet $\mathcal{N}=2$ superconformal symmetry gives rise as usual to space-time supersymmetry. Since it is present only at $R=2 / Q$, we call it the supersymmetric radius. $^{2}$ In other words, the radius changing operator is not an $N=2$ primary. For

\footnotetext{
${ }^{2}$ The stability of the linear dilaton background away from the supersymmetric radius has been recently discussed in [18].
} 
the $(2 n+2)$-dimensional superstrings we can construct $2^{n+2}$ candidates for space-time supercurrents in the $-\frac{1}{2}$ picture

$$
q \sim e^{-\frac{\phi}{2}+\frac{i}{2}\left( \pm H \pm H^{1} \pm \ldots \pm H^{n} \pm Q x\right)}
$$

with the usual bosonization of the superghosts $\beta=\partial \xi e^{-\phi}$ and $\gamma=e^{\phi} \eta$.

Only $2^{n}$ of them are mutually local and BRST invariant. Combining the left and right sectors, we can realize a space-time supersymmetry algebra with $2^{n+1}$ real supercharges that close on the $S O(d)$ translation along the flat $\mathbb{R}^{d}$ part of the spacetime $(d=2 n)$. In the case of even $n$, the supercharges are in different $S O(d)$ spinor representations, while if $n$ is odd they are in the same spinor representation. The circle on which $x$ is compactified is related to the R-symmetry: the momentum along the circle corresponds to the R-charge and is measured by the affine current

$$
J_{R}=\frac{2 i}{Q(n)} \partial x
$$

In the RNS formalism, space-time supersymmetry only closes up to picture changes. We will need to consider supercharges in the $+\frac{1}{2}$ picture as well, so we will make use of the picture raising operator $Z_{+}$

$$
Z_{+}=\left\{Q_{B}, \xi\right\}=2 \partial \phi b \eta e^{2 \phi}+e^{\phi}\left(G^{+}+G^{-}\right)+2 b \partial \eta e^{2 \phi}+\partial b \eta e^{2 \phi}+c \partial \xi .
$$

We will return later to the supersymmetry algebra in the various dimensions and show explicitely its current algebra case by case.

\subsection{Spectrum}

In this section, we will collect some useful results about the spectrum in various dimensions [19, that we will compare to the pure spinor covariant cohomology computation.

Consider first various general features of the superstring, which are valid in all non-critical dimensions on the backgrounds (3.1). The RNS computation of the spectrum follows an indirect path, since an explicit BRST analysis has been done only in the $d=0$ case. It can be done in three steps:

1) Identify the physical space-time supercharges and GSO project the vertex operators.

2) Impose the on-shell condition $\Delta=1$ and the Dirac equation.

3) Impose the level matching conditions on the operators with the same momentum in the noncompact Liouville direction $\varphi$.

4) Require that the vertex operators are all mutually local with respect to each other.

The bosonic part of the lowest level spectrum is what, in a familiar ten dimensional language, we would have called the graviton, the dilaton and the $B$-field 
plus the appropriate odd dimensional RR field strengths ${ }^{3}$ and a new character, the tachyon, which in the non-critical case is non tachyonic and survives the GSO projection. However, because the theory is compactified on a small circle, the analysis of the spectrum from the $d+2$ dimensional point of view is misleading and we should think of them as on-shell string modes in $d+1$ dimensions with certain winding and momentum around the circle. In this picture, all the $(d+1)$-dimensional modes are massive and the lowest lying state is always the tachyon (in $d+1=5$ dimensions the tachyon is massless). On the other hand, the spectrum must arrange itself into representations of the space-time symmetries, namely the super-Poincaré group acting on the flat $\mathbb{R}^{1, d-1}$ part of the space-time. We have to fit the $(d+1)$-dimensional modes into supersymmetry multiplets of $d$ dimensions. There are two crucial features here:

i) From the $(d+1)$-dimensional point of view the string modes are on-shell: the mass in their dispersion relation is fixed, because we are reducing on the compact direction $x{ }^{4}$ When we further reduce these modes along the Liouville direction down to $d$ dimensions, however, they arrange themselves into off-shell $d$ dimensional supermultiplets. Because the momentum in the noncompact Liouville direction $\varphi$ is continuous, in fact, the mass that appears in the dispersion relation for the $d-$ dimensional momentum $k_{\mu}$ is continuous, above a certain mass gap.

ii) The different winding and momentum modes of the same parent $(d+2)$ dimensional RR field strength fit into different $d$-dimensional supermultiplets.

There are two kinds of vertex operators in the theory: the normalizable modes are particles propagating in the bulk of the linear dilaton and they corresponds to states in the holographic dual theory (in the AdS/CFT sense); the non-normalizable vertex operators instead have a wavefunction exponentially supported in the weak coupling region and they correspond to operators in the dual theory. We will be interested in computing the deformations of the worldsheet lagrangian, corresponding to variations of the string background, so we will focus just on the non-normalizable operators. In particular, the ones which are chiral primary fields of the $N=2$ worldsheet superalgebra can be added to the action without breaking the $N=2$ superconformal symmetry itself. This means that they correspond in the dual theory to observables that preserve space-time supersymmetry and they necessarily fit into off-shell short representations of the space-time supersymmetry.

Let us now summarize the symmetries and the spectrum of the non-critical superstring (to be concrete we will only consider type IIB) on the background $\mathbb{R}^{d-1,1} \times \mathbb{R}_{\varphi} \times U(1)_{x}$ in the various dimensions. We always find two kinds of off-shell supermultiplets, a gauge multiplet, to which the tachyon belongs, and a supergravity multiplet. At the end, we will discuss how the holographic picture of the background is realized on this spectrum.

\footnotetext{
${ }^{3}$ In $d+2$ dimension, if there is a $F_{+}^{(d+2 / 2)}$ form it is also self dual.

${ }^{4}$ The dispersion relation comes from the condition $\Delta=1$ for on-shell vertex operators.
} 


\section{$d=0$}

This is the so called two-dimensional non-critical superstring. In this case there is no Lorentz symmetry, but only $\mathcal{N}=2$ supersymmetry in zero dimensions with a $U(1)$ R-symmetry. There is no supergravity sector in this case, the graviton in fact is just a discrete state. We only have the gauge multiplet, containing the RR scalar potential $C$, the tachyon $T$ and two real fermions, ${ }^{5}$ for a total of $2 \oplus 2$ degrees of freedom.

$$
d=2
$$

We have $S O(1,1)$ Lorentz symmetry acting on the flat $\mathbb{R}^{1,1}$ and in the type IIB case we find $\mathcal{N}=(4,0)$ supersymmetry with a $U(1) \times \mathbb{Z}_{2}$ R-symmetry. We still have two supermultiplets. The gauge supermultiplet, containing the tachyon, has $4 \oplus 4$ off-shell degrees of freedom. Then we have the two-dimensional supergravity multiplet, containing $8 \oplus 8$ degrees of freedom.

\section{$d=4$}

In this case we have $\mathcal{N}=2$ super-Poincaré $S O(1,3)$ symmetry acting on the flat $\mathbb{R}^{1,3}$ part of the space-time. In addition we have also a $U(1)$ R-symmetry. The spectrum contains an $\mathcal{N}=2$ off-shell gauge multiplet, which contains the tachyon and has $8 \oplus 8$ degrees of freedom. Then we have the $\mathcal{N}=2$ supergravity multiplet with $32 \oplus 32$ states.

We collect the various spectra in the following table:

$$
\begin{array}{cccc} 
& d=0 & d=2 & d=4 \\
\text { gauge: } & 2 \oplus 2 & 4 \oplus 4 & 8 \oplus 8 \\
\text { supergravity: } & - & 8 \oplus 8 & 32 \oplus 32
\end{array}
$$

Consider the holographic interpretation in the $d=4$ case [20]. The Liouville direction $\varphi$ is the holographic direction. At the weak coupling end of the space $\varphi=-\infty$ a four dimensional non-gravitational theory lives, which is non local and is called Little String Theory. Its low energy limit is a particular $\mathcal{N}=2$ supersymmetric gauge theory, the $S U(2)$ Seiberg-Witten theory at the singular point in the moduli space of vacua where a monopole becomes massless. This system can also be realized as type IIB string theory on the conifold at vanishing string coupling, in which case we know that this is the dual gauge theory.

At the monopole point, the gauge theory is abelian and its field content consists of an $\mathcal{N}=2$ gauge multiplet and a massless hypermultiplet. The latter contains

\footnotetext{
${ }^{5}$ From the two dimensional point of view the tachyon only gives rise to winding modes and usually it is not included in the propagating spectrum of the two dimensional non-critical string. But here we are classifying the spectrum according to zero dimensional supersymmetry, so we include it in the supermultiplet. Moreover, the RR potential is a two dimensional chiral boson and the two fermions are chiral.
} 
the massless monopole (which can be realized as a D3-brane wrapping the vanishing 3 -cycles of the conifold). In the dual perturbative string spectrum we can only see the off-shell vector multiplet, which corresponds precisely to the supermultiplet with $8 \oplus 8$ degrees of freedom to which the tachyon belongs.

A similar picture exist in the lower dimensions, corresponding to type IIB superstring on a higher dimensional conifolds at vanishing string coupling. These give rise to lower dimensional Little String Theories. The vector multiplet in their low energy spectrum corresponds to the gauge multiplet (in which the "non-tachyonic" tachyon sits) of the superstring in the linear dilaton background.

\section{Two-dimensional superstrings}

In this section we will construct the pure spinor superstring in the linear dilaton background

$$
\mathbb{R}_{\varphi} \times U(1)_{x}
$$

This theory has two bosonic dimensions, the dilaton direction $\varphi$ and the $x$ direction. The latter is compactified at the supersymmetric radius $R=2 / Q$, where $Q=2$ is the Liouville background charge. Since there are no transverse directions $(d=0)$, this two dimensional case is the easiest case for explaining the construction of the corresponding pure spinor theory.

The strategy for the construction of the pure spinor non-critical superstring is that we first map the RNS worldsheet variables to a patch of the pure spinor space and then we reconstruct the covariant formulation. As we will see, an important feature of the model is that it is naturally embedded in a larger superspace, that we will eventually reduce to the physical one. This will be a feature that allows to generalize the non-critical pure spinor action to other backgrounds with a larger amount of supersymmetries.

We first recall some facts about the RNS computation of the spectrum and supersymmetry algebra [21, 22, 23]. Then we will introduce the map to the pure spinor variables and reconstruct the full covariant theory. We will compute the cohomology and show that it agrees to the RNS spectrum. Eventually, we will present some speculations about the possible generalization to other non-critical two dimensional backgrounds.

\subsection{The RNS superstring}

The supersymmetry structure of the model is that of a zero-dimensional space-time. Consider the holomorphic part. The addition of the antiholomorphic sector in order to get the closed superstring is straightforward. 
The model has one real supercharge, and we can choose the BRST invariant supercurrent $q_{+}(z)$ in the $-\frac{1}{2}$ picture

$$
q_{+}=e^{-\frac{1}{2} \phi+\frac{i}{2} H-i x} .
$$

The corresponding supercharge $Q$ is given by

$$
Q_{+}=\oint e^{-\frac{1}{2} \phi+\frac{i}{2} H-i x}
$$

and is nilpotent $Q_{+}^{2}=0$, as we expect from the fact that we have no transverse space and hence zero dimensional supersymmetry. ${ }^{6}$ We have another physical supercurrent $q_{-}=e^{-\frac{1}{2} \phi-\frac{i}{2} H+i x}$ which is nonlocal with respect to $q_{+}$. The choice of the $q_{+}, \bar{q}_{+}$gives type IIB, while the pairing $q_{+}, \bar{q}_{-}$gives type IIA. We will only consider the former case.

A basic feature of the map from the RNS to the pure spinor variables is that it requires doubling the superspace. In [4] it has been noted the existence of another supercurrent $q_{\dot{+}}(z)$ of the form

$$
q_{\dot{+}}=e^{-\phi / 2+i H / 2+i x} .
$$

The supercurrents $q_{+}$and $q_{+}$are mutually local and the latter is a conserved current as well, so the correponding charge is conserved. However, while $q_{+}$is BRST invariant, $q_{+}$is not. Indeed, the model has only one physical real supersymmetry. The supercharges satisfy

$$
\left\{Q_{+}, Q_{\dot{+}}\right\}=\oint e^{-\phi+i H} .
$$

However, (4.4) is not a supersymmetry algebra. Recalling that in the RNS formalism supersymmetry only closes up to picture changing, we can picture raise the physical supercurrent $q_{+}$to the $\frac{1}{2}$ picture

$$
q_{+}^{(+1 / 2)}=b \eta e^{3 \phi / 2+i H / 2-i x}+i \partial(\varphi+i x+i Q H) e^{\phi / 2-i H / 2-i x},
$$

and compute the OPE between the physical supercurrent $q_{+}^{(+1 / 2)}$ and the unphysical one in the $-\frac{1}{2}$ picture

$$
q_{+}^{(+1 / 2)}(z) q_{+}^{(-1 / 2)}(0) \sim \frac{Q}{z^{2}}+\frac{i}{z} \partial(\varphi+i x+i Q H)(0),
$$

which is indeed not a supersymmetry current algebra, due to the presence of the double pole. This anomalous term is proportional to the Liouville background charge $Q=2$.

As we will see, working in a doubled superspace in the pure spinor variables will require an appropriate projection to the physical superspace. However, the doubled superspace will allow to study pure spinor superstrings in two-dimensional backgrounds with four supercharges, that is twice as many supersymmetries as in the linear dilaton background.

\footnotetext{
${ }^{6}$ This theory is realized as type II superstring on a Calabi-Yau fivefold in the limit of zero string coupling.
} 


\subsection{Multiplet spectra: RNS analysis}

In the following we will construct the multiplet spectra of the RNS superstring on the $\mathbb{R}_{\varphi} \times U(1)_{x}$ background. We first consider the holomorphic sector as a building block for the closed superstring multiplet.

As we anticipated in Section 8 , we will consider non-normalizable vertex operators only and we normalize their Liouville dependent part as

$$
V \sim e^{\beta \varphi}=e^{\frac{Q}{2} \varphi} e^{-E \varphi} \equiv g_{S}(\varphi) e^{-E \varphi},
$$

such that the corresponding wavefunction $\Psi(E) \sim e^{-E \varphi}$ is localized in the weak coupling region $g_{s}(\varphi) \rightarrow 0$. These operators satisfy the Seiberg bound 24]

$$
\operatorname{Re} \beta \leq \frac{Q}{2},
$$

There are also operators with complex $\beta=Q / 2+i k_{\varphi}$ whose imaginary part is the momentum of the wavefunction of a particle moving in the $\varphi$ direction. They correspond to propagating particles that can be scattered.

\subsubsection{Holomorphic sector}

We begin with the NS sector.

NS sector

The tachyon vertex operator in the -1 picture is

$$
T=e^{-\phi+i p x+\beta \varphi} .
$$

The condition for $T$ to have weight $\Delta(T)=1$ is

$$
p^{2}-\beta(\beta-Q)=1,
$$

where $Q=2$. The requirement for mutual locality of $T$ with the supercurrent $q_{+}$ (GSO projection) reads $p Q \in 2 \mathbb{Z}+1$. Note that this condition also implies that $T$ is mutualy local with $q_{\dot{+}}$. The lowest lying states have $p= \pm \frac{1}{Q}$. The operator

$$
T_{ \pm}=e^{-\phi+\frac{1}{Q}(\varphi \pm i x)},
$$

is a worldsheet (anti)chiral primary (annihilated by $G^{ \pm}$) of $\Delta_{\text {matter }}\left(T_{ \pm}\right)= \pm \frac{p}{2}=\frac{1}{2}$, with space-time R-charge $R= \pm \frac{1}{2}$. Both are non-normalizable operators as their Liouville momentum satisfies $\beta<\frac{Q}{2}$. Note that the OPE of $T^{+}$and $T^{-}$has a branch cut, so they are not both mutually local.

The NS sector may also contain a vector of the form

$$
V_{ \pm}=e^{-\phi+i \epsilon H+i p x+\beta \varphi}, \quad \epsilon= \pm 1 .
$$


The weight requirement is $p^{2}=\beta(\beta-Q)$ and the mutual locality condition with the supercharge requires $p Q \in 2 \mathbb{Z}$. In order to be a primary of the worldsheet $N=1 \mathrm{SCA}$ (i.e., having no double poles with the supercurrent $G_{m}$ ) we need that $\beta=\epsilon p+Q$, which together with the weight one requirement imply that $p=0$ and $\beta=Q$. This violates the Seiberg bound and the operator is normalizable, so it does not fit into space-time short supermultiplets.

\section{Ramond sector}

The Ramond sector operator in the $-\frac{1}{2}$ picture reads

$$
R_{ \pm}=e^{-\phi / 2+i \epsilon H / 2+i p x+\beta x} .
$$

The weight one condition is $p^{2}-\beta(\beta-Q)=1$, and the mutual locality with the supercharge implies that $2 p Q \in 4 \mathbb{Z}+\epsilon-1$. The Dirac condition

$$
\oint \gamma\left(G^{+}+G^{-}\right) R=0 .
$$

is $\beta=\epsilon p+\frac{Q}{2}$, which satisfies the weight one condition identically.

Consider the lowest space-time R-charge states. At $\epsilon=+1$, the vertex operator with $p=0$ saturates the bound $\beta=\frac{Q}{2}$. When $p=-1$ we get

$$
R_{+}=e^{-\phi / 2+i H / 2-i x},
$$

which is the supercharge $q_{+}$.

At $\epsilon=-1$ and $p=\frac{1}{2}$ we find the supersymmetric partner $R_{-}$of the tachyon $T_{-}$

$$
R_{-}=e^{-\phi / 2-i H / 2+(\varphi+i x) / Q} .
$$

$T_{-}$and $R_{-}$are of course mutually local.

\subsubsection{Closed superstring}

We consider the Type IIB theory with the supercharges $Q_{+}$and $\bar{Q}_{+}$from the holomorphic and anti-holomorphic sectors, respectively. In order to construct the $\mathcal{N}=2$ $d=0$ supersymmetric multiplet we use the results of the holomorphic sector from above.

The bosons of the closed string multiplet are the NS-NS operator $T^{-} \bar{T}^{-}$and the $\mathrm{R}-\mathrm{R}$ operator $R_{-} \bar{R}_{-}$. The fermions are the R-NS and NS-R operators $R_{-} \bar{T}_{-}$and $T_{-} \bar{R}_{-}$. They are arranged in the supermultiplet

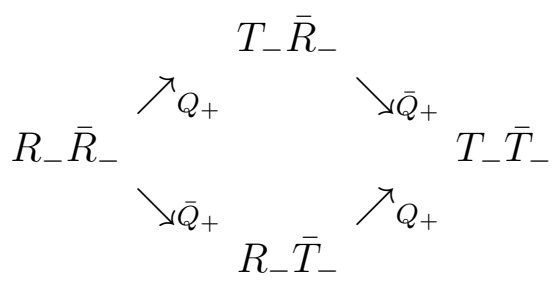

for a total of $2 \oplus 2$ degrees of freedom. 


\subsection{Pure spinor variables}

The RNS superstring has four bosonic fields: $x, \varphi, \beta$ and $\gamma$, and four fermionic fields: $\psi_{x}, \psi_{l}, b$ and $c$. In the following we will map them to four bosonic and four fermionic fields, which will be the pure spinor superstring variables.

The fermionic variables are the dimension zero fermionic coordinates of the doubled superspace $\left(\theta^{+}, \theta^{\dot{+}}\right)$ and their dimension one conjugate momenta $\left(p_{+}, p_{\dot{+}}\right)$

$$
\begin{array}{lll}
p_{+}=b \eta e^{\frac{3}{2} \phi+\frac{i}{2} H-i x}, & \theta^{+}=c \xi e^{-\frac{3}{2} \phi-\frac{i}{2} H+i x}, \\
p_{\dot{+}}=e^{-\frac{1}{2} \phi+\frac{i}{2} H+i x}, & \theta^{\dot{+}}=e^{\frac{1}{2} \phi-\frac{i}{2} H-i x} .
\end{array}
$$

Note that $\left(\theta^{+}, p_{+}\right)$are BRST invariant while $\left(\theta^{\dot{+}}, p_{\dot{+}}\right)$ are not BRST invariant.

These fermionic variables have the free field OPE's

$$
p_{+}(z) \theta^{+}(0) \sim \frac{1}{z}, \quad p_{\dot{+}}(z) \theta^{\dot{+}}(0) \sim \frac{1}{z},
$$

and all the other OPE's vanish.

Consider next the bosonic variables. We construct a map analogous to the one we used for the ten dimensional critical superstring in Section 2.1

$$
\eta=e^{\tilde{\phi}+\tilde{\kappa}} p_{+}, \quad b=e^{(\tilde{\phi}-\tilde{\kappa}) / 2} p_{+},
$$

$b$ and $\eta$ are the RNS fields and we introduced two new variables $\tilde{\phi}$ and $\tilde{\kappa}$, which we will relate to the pure spinor variables.

Their OPE's are

$$
\begin{aligned}
& \tilde{\phi}(z) \tilde{\phi}(0) \sim-\log z, \\
& \tilde{\kappa}(z) \tilde{\kappa}(0) \sim \log z,
\end{aligned}
$$

and $\tilde{\phi}(z) \tilde{\kappa}(0) \sim 0$. It can be easily verified that the OPE's of $\tilde{\phi}$ and $\tilde{\kappa}$ with either the fermionic momenta or the fermionic coordinates are all non-singular.

We can express $\tilde{\phi}$ and $\tilde{\kappa}$ via the RNS variables as

$$
\begin{aligned}
\tilde{\phi} & =-\frac{9}{4} \phi-\frac{3}{4} i H+\frac{3}{2} i x-\kappa+\frac{1}{2} \chi, \\
\tilde{\kappa} & =\frac{3}{4} \phi+\frac{i}{4} H-\frac{i}{2} x+\kappa+\frac{1}{2} \chi .
\end{aligned}
$$

However, the OPE's of $x$ with the fermionic momenta and fermionic coordinates as well as with $\tilde{\phi}$ and $\tilde{\kappa}$ are singular. In order to fix this, we shift the $x$ coordinate and define

$$
x^{\prime}=x+i \phi-H,
$$

whose OPE is

$$
x^{\prime}(z) x^{\prime}(0) \sim-\log z .
$$


Using these new variables the RNS energy-momentum tensor $T=T_{\mathrm{m}}+T_{\text {gh }}$ is mapped to

$$
\begin{aligned}
T= & -\frac{1}{2}(\partial \varphi)^{2}-\frac{1}{2}\left(\partial x^{\prime}\right)^{2}+\partial^{2}\left(\varphi-i x^{\prime}\right)-p_{+} \partial \theta^{+}-p_{\dot{+}} \partial \theta^{\dot{+}}- \\
& -\frac{1}{2}(\partial \tilde{\phi})^{2}+\frac{1}{2}(\partial \tilde{\kappa})^{2}+\partial^{2}(\tilde{\phi}+\tilde{\kappa}) .
\end{aligned}
$$

The pure spinor variable $\lambda^{+}$and its conjugate momentum $w_{+}$are recovered in terms of $\tilde{\phi}$ and $\tilde{\kappa}$ as an ordinary beta gamma system

$$
\lambda^{+}=e^{\tilde{\phi}+\tilde{\kappa}}, \quad w_{+}=\partial \tilde{\kappa} e^{-\tilde{\phi}-\tilde{\kappa}} .
$$

Their OPE reads

$$
w_{+}(z) \lambda^{+}(0) \sim \frac{1}{z}
$$

The pure spinor variable $\lambda^{+}$parameterizes the patch $\left(\lambda^{+} \neq 0, \lambda^{\dot{+}}=0\right)$ of the complex dimension one pure spinor space

$$
\lambda^{+} \lambda^{\dot{+}}=0
$$

It will be a generic feature in all dimensions that the map takes the RNS variables to the pure spinor variables on a patch of the pure spinor space.

The total central charge of the theory still vanishes

$$
c=(1-12)_{\left\{x^{\prime}\right\}}+(1+12)_{\{\varphi\}}+(-2)_{\left\{p_{+} \theta^{+}\right\}}+(-2)_{\left\{p_{\dot{+}} \theta^{+}\right\}}+(2)_{\left\{\lambda^{+} w_{+}\right\}}=0 .
$$

The pure spinor variables form a curved beta-gamma system. The pure spinor energy-momentum tensor, that we get from $T_{\tilde{\phi}, \tilde{\kappa}}$ on the patch is not simply $T_{\left(\lambda^{+}, w_{+}\right)}=$ $w_{+} \partial \lambda^{+}$. Rather, it has an additional term as in the critical superstring case

$$
T_{\left(\lambda^{+}, w_{+}\right)}=w_{+} \partial \lambda^{+}-\frac{1}{2} \partial^{2} \log \Omega
$$

where $\Omega=e^{-3(\tilde{\phi}+\tilde{\kappa})}=\left(\lambda^{+}\right)^{-3}$. This arises from the top form on pure spinor space, which reads on the patch $\left(\lambda^{+} \neq 0, \lambda^{\dot{+}}=0\right)$

$$
\Omega\left(\lambda^{+}\right)=\frac{d \lambda^{+}}{\left(\lambda^{+}\right)^{3}}
$$

Mapping the RNS saturation rule on the sphere to the pure spinor variables one gets a requirement for an insertion of $\left(\lambda^{+}\right)^{3}$, which is consistent with the measure corresponding to the top form we obtained. This ghost number three insertion, required for a nonvanishing amplitude, will be the same in all different non-critical dimension and actually coincides with the ten dimensional saturation rule, we derived in Section 2.1. 
We can write the pure spinor stress tensor in a covariant way by

$$
\begin{aligned}
T= & -\frac{1}{2}(\partial \varphi)^{2}-\frac{1}{2}(\partial x)^{2}+\partial^{2}(\varphi-i x)-p_{I} \partial \theta^{I}+ \\
& +w_{I} \partial \lambda^{I}-\frac{1}{2} \partial^{2} \log \Omega(\lambda)
\end{aligned}
$$

where $I=+, \dot{+}$, and we renamed $x^{\prime}$ as $x$ for the simplicity of notation.

The matter part of this stress tensor can be derived from the pure spinor action

$$
S=\frac{1}{2 \pi \alpha^{\prime}} \int d^{2} z\left(\frac{1}{2} \partial x \bar{\partial} x+\frac{1}{2} \partial \varphi \bar{\partial} \varphi+p_{I} \bar{\partial} \theta^{I}\right)-\frac{Q}{2} \int d^{2} z r^{(2)}(\varphi-i x)
$$

where the last term is the Fradkin-Tseytlin term that couples the space-time linear dilaton to the worldsheet curvature. For the consistency of the FT term we have to compactify the $x$ direction on a circle of radius $R=2 / Q$, which in fact is the supersymmetric radius we already know from RNS analysis. Note that the hermiticity property of the action and the stress tensor implies that the hermiticity properties of the variables is not the naive one [25].

\subsubsection{Supersymmetry structure}

An important ingredient in the construction of the pure spinor non-critical superstring is the supersymmetry algebra and the superspace structure. Let us construct the pure spinor superstring. Recall that the map from the RNS variables to the pure spinor variables imposed on us the introduction of an additional fermionic coordinate and its conjugate momentum $\left(\theta^{\dot{+}}, p_{\dot{+}}\right)$, which are not physical. The RNS OPE between the supercharges in (4.6) is mapped on the pure spinor side to

$$
q_{+}(z) q_{\dot{+}}(0) \sim \frac{Q}{z^{2}}+\frac{\partial(\varphi-i x)(0)}{z}
$$

where we denoted $x^{\prime}$ by $x$ for simplicity of notation. The corresponding algebra of the superderivatives has the opposite sign as usual

$$
d_{+}(z) d_{\dot{+}}(0) \sim-\frac{Q}{z^{2}}-\frac{\partial(\varphi-i x)(0)}{z} .
$$

We introduce GS-like constraints that reproduce this algebra

$$
\begin{aligned}
& d_{+}=p_{+}-\frac{1}{2} \theta^{\dot{+}} \partial(\varphi-i x)+\frac{1}{2} Q \partial \theta^{\dot{+}} \\
& d_{\dot{+}}=p_{\dot{+}}-\frac{1}{2} \theta^{+} \partial(\varphi-i x)-\frac{1}{2} Q \partial \theta^{+}
\end{aligned}
$$

where the crucial difference with respect to the flat background is the last term, proportional to the background charge $Q$. This term is responsible for the double pole in the algebra, which signals the breaking of the two-dimensional flat supersymmetry 
algebra to the physical zero-dimensional supersymmetry algebra of the linear dilaton background. We introduce the compact notation

$$
d_{I}=p_{I}-\frac{1}{2} \tau_{I J} \theta^{J} \partial(\varphi-i x)+\frac{Q}{2} \epsilon_{I J} \partial \theta^{J}
$$

where $I=+, \dot{+}$. The two dimensional matrices $\tau_{I J}=\sigma_{I J}^{1}$ (the Pauli matrix) and $\epsilon_{I J}$, the usual antisymmetric tensor normalized as $\epsilon_{+\dot{+}}=1$, act in the following on the index $I$, by which we denote the physical supercoordinates, $I=+$, and the unphysical ones, $I=\dot{+}$.

\subsubsection{Cohomology}

An essential ingredient of the pure spinor formulation is the BRST operator, that in the two dimensional background is

$$
Q_{B}=\oint \lambda^{I} d_{I}
$$

where $I=+, \dot{+}$. In the critical superstring in flat ten dimensions, all the superderivatives in the BRST charge $Q=\oint \lambda^{\alpha} d_{\alpha}$ are physical. The first issue that needs to be dealt with in the linear dilaton background (and essentially in any case with reduced supersymmetry) is whether to include in (4.37) only the physical superderivatives or also the unphysical ones. The strategy we follow is to include always in the BRST operator all the $d^{\prime}$ 's, in our case both $d_{+}$and $d_{\dot{+}}$, and compute the cohomology thereof. In a second step, since we are interested in the space-time supersymmetry multiplets, we will consider only the part of the cohomology that contains the variables that realize the supersymmetry current algebra, in this case $\theta^{+}$. This will reproduce the RNS computation of the short supermultiplets. Recall that the full current algebra of the $d$ 's is anomalous, i.e. it has a double pole in the OPE between $d_{+}$and $d_{\dot{+}}$ : the non anomalous subalgebra is in fact the space-time supersymmetry algebra, in our case the zero dimensional supersymmetry $d_{+}^{2}=0$.

The two dimensional pure spinor constraint (4.28) is sufficient to prove the nilpotency of the BRST charge $Q_{B}$. Looking at the algebra (4.34) we see that $Q_{B}^{2}=0$, provided the following conditions are satisfied

$$
\lambda^{+} \lambda^{\dot{+}}=0, \quad \partial \lambda^{+} \lambda^{\dot{+}}-\lambda^{+} \partial \lambda^{\dot{+}}=0 .
$$

The first condition is the pure spinor constraint itself. The derivative condition is a consequence of the first condition. There are various ways to see this [26]. The simplest is to expand the condition $\lambda^{+} \lambda^{\dot{+}}=0$ in modes

$$
\lambda_{0}^{+} \lambda_{0}^{\dot{+}}=0, \quad \lambda_{0}^{+} \lambda_{1}^{\dot{+}}+\lambda_{1}^{+} \lambda_{0}^{\dot{+}}=0, \quad \ldots
$$

Then for a solution of the zero mode $\lambda_{0}^{+} \neq 0$, the second equation implies $\lambda_{0}^{+} \lambda_{1}^{\dot{+}}=0$ and so on. Hence, the derivatives separately vanish, $\lambda^{+} \partial \lambda^{+}=0$. The same argument 
on the other solution $\lambda_{0}^{+} \neq 0$ implies that $\partial \lambda^{+} \lambda^{+}=0$. In particular, we see that the two derivative terms in (4.38) vanish separately and the BRST charge is nilpotent. ${ }^{7}$

To simplify the computation of the cohomology it is convenient to introduce the new notation $Z=\varphi+i x$ and $\bar{Z}=\varphi-i x$ with $\mathrm{OPE}$

$$
Z(z) \bar{Z}(0) \sim-2 \log z
$$

so that the GS-like contraints read

$$
d_{+}=p_{+}-\theta^{\dot{ }} \partial \bar{Z}+Q \partial \theta^{\dot{+}}, \quad d_{\dot{+}}=p_{\dot{+}},
$$

whose OPE is still (4.34). The physical states are the vertex operators at ghost number one and weight zero, we find two different kinds of operators. The first is analogous to the usual ten dimensional vertex operator. In general this contains the off-shell $d$ dimensional supergravity multiplet, even if in the particular $d=0$ there are no such multiplets. The second type is new and peculiar to the linear dilaton background. It contains what we called the "gauge multiplet" in the rehearsal of the spectrum (3.10), including the tachyon, which in fact is the peculiarity of the linear dilaton background also in the RNS.

Usually in the pure spinor formalism, massless states are given by the zero weight ghost number one vertex operator. Since the only worldsheet fields with weight zero are the zero modes of the fields $\varphi, x$ and $\theta^{I}$, this vertex operator has to be expressed by these alone with a single power of $\lambda^{I}$ so that it be of ghost number one. Hence the vertex operator is of the form

$$
\mathcal{V}^{(1)}=\lambda^{I} A_{I}\left(\bar{Z}, \theta^{J}\right)
$$

where

$$
A_{I}\left(\bar{Z}, \theta^{J}\right)=B_{I}(\bar{Z})+\theta^{J} C_{I J}(\bar{Z})+\theta^{I} \theta^{J} D_{I J K}(\bar{Z}) .
$$

Next we have to require that it be BRST closed when the pure spinor constraint $\lambda^{+} \lambda^{+}=0$ is imposed but not solved. This leaves us with just

$$
\begin{aligned}
& A_{+}=B_{+}+\theta^{\dot{+}} C_{+\dot{+}}-2 i \theta^{+} \theta^{\dot{ }} \partial_{Z} B_{+}, \\
& A_{\dot{+}}=B_{\dot{+}}+\theta^{+} C_{\dot{+}+} .
\end{aligned}
$$

These superfields still have some gauge freedom given by zero weight ghost number one $Q_{B}$-exact terms. Parameterizing the general weight zero ghost number zero gauge transformation superfield as

$$
\Omega=\eta+\theta^{+} \xi_{+}+\theta^{\dot{+}} \xi_{+}+2 \theta^{+} \theta^{\dot{+}} \Lambda_{+\dot{+}}
$$

\footnotetext{
${ }^{7}$ Another way to prove that the derivatives vanish separately is to use the Ward identities coming from the Lorentz current and the ghost current OPE's [26].
} 
so that

$$
\begin{aligned}
\delta \mathcal{V}^{(1)}= & Q_{B} \Omega^{(0)}=\lambda^{+}\left(\xi_{+}+2 \theta^{\dot{+}} \Lambda_{+\dot{+}}+2 i \theta^{\dot{+}} \partial_{Z} \eta-2 i \theta^{+} \theta^{\dot{+}} \partial_{Z} \xi_{+}\right)+ \\
& +\lambda^{\dot{+}}\left(\xi_{\dot{+}}-2 \theta^{+} \Lambda_{+\dot{+}}\right)
\end{aligned}
$$

the gauge transformations of the components of the vertex operator are given by

$$
\begin{array}{ll}
B_{+} \rightarrow B_{+}+\xi_{+}, & C_{+\dot{+}} \rightarrow C_{+\dot{+}}+2 \Lambda_{+\dot{+}}+2 i \partial_{Z} \eta, \\
B_{\dot{+}} \rightarrow B_{\dot{+}}+\xi_{\dot{+}}, & C_{\dot{+}+} \rightarrow C_{\dot{+}+}-2 \Lambda_{+\dot{+}},
\end{array}
$$

so that the entire vertex operator is pure gauge and this sector of the cohomology is trivial.

The second type of vertex operator is peculiar to the linear dilaton background. Due to the term $\partial^{2} \bar{Z}$ appearing in the stress tensor (4.31), we see that the operator $\exp \left(-\frac{Z}{Q}\right)$ has weight -1 . We can obtain again a weight zero ghost number one operator by the following procedure. Consider the weight one ghost number one operator, which in the ten dimensional background corresponds to the first massive level 13

$$
\mathcal{U}^{(1)}=\partial \lambda^{I} A_{I}+\lambda^{I} \partial \theta^{J} B_{I J}+\lambda^{I} d_{J} C_{I}^{J}+\lambda^{I} \Pi^{\bar{Z}} H_{I}+\lambda^{+} J_{+}^{+} F_{++}^{+}+\lambda^{\dot{+}} J_{\dot{+}}^{\dot{+}} F_{\dot{+} \dot{+}}^{\dot{+}},
$$

where $A_{I}, B_{I J}, C_{I}, H_{I}^{J}, F_{I}$, for $I, J=+, \dot{+}$, are generic superfields constructed with the $\theta^{I}$ coordinates and $J=w_{I} \lambda^{I}$ is the pure spinor $U(1)$ current. However now we restrict the wavefunctions in these superfields to be $e^{-Z / Q}$, so the total weight of the operator (4.47) is zero. The gauge invariance of this vertex operator is

$$
\delta \mathcal{U}^{(1)}=Q_{B} \Lambda^{(0)},
$$

where $\Lambda^{(0)}$ is a weight one, ghost number zero vertex operator constructed out of the

$$
\Lambda^{(0)}=\partial \theta^{I} \Omega_{I}+\partial(\varphi-i x) \Gamma+p_{I} \Lambda^{I}+J \Phi,
$$

and again $\Omega_{I}, \Gamma, \Lambda, \Phi$ are generic superfields, whose wavefunctions are chosen to be $e^{-Z / Q}$, such that the total weight of $\Lambda^{(0)}$ vanishes. We postpone the details about the cohomology computation to the appendix. The result is that the only operator that survives in the cohomology is

$$
\mathcal{U}^{(1)}=\left(\lambda^{\dot{+}} \partial \theta^{+}\right) B_{\dot{+}+}, \quad D_{\dot{+}} B_{\dot{+}+}=0 .
$$

The chiral superfield $B_{\dot{+}+}=T+\theta^{+} R$ contains $1 \oplus 1$ states, $T$ being a real boson and $R$ a Majorana-Weyl fermion. Since in the pure spinor formalism we do not have to worry about GSO projections, the closed string spectrum is just given by the left-right producet of the open string one

$$
\text { closed }=\text { open } \otimes \overline{\text { open }},
$$

Therefore we find $2 \oplus 2$ states, reproducing the RNS result for the "gauge" supermultiplet. 


\subsubsection{Curved non-critical backgrounds}

In this section we suggest a generalization of the linear dilaton action to a generic two dimensional non-critical background with at most four real supercharges.

Even if the linear dilaton background has only zero dimensional supersymmetry, we introduce the momenta that will be useful when generalizing the model to backgrounds with extra supersymmetry

$$
\Pi^{\varphi}=\partial \varphi+\frac{1}{2} \tau_{I J} \theta^{I} \partial \theta^{J}, \quad \Pi^{x}=\partial x-\frac{i}{2} \tau_{I J} \theta^{I} \partial \theta^{J} .
$$

We have the following OPE's

$$
d_{I}(z) \Pi^{\varphi}(0) \sim \frac{\tau_{I J} \partial \theta^{J}(0)}{z}, \quad d_{I}(z) \Pi^{x}(0) \sim-i \frac{\tau_{I J} \partial \theta^{J}(0)}{z} .
$$

The stress tensor (4.31) can be cast in the following form

$$
\begin{gathered}
T=-\frac{1}{2} \Pi^{m} \Pi^{n} \eta_{m n}+\frac{Q}{2} \epsilon_{I J} \partial \theta^{I} \partial \theta^{J}-d_{I} \partial \theta^{I}+\frac{Q}{2} \partial^{2}\left(\Pi^{\phi}-i \Pi^{x}\right) \\
+w_{I} \partial \lambda^{I}-\frac{1}{2} \partial^{2} \log \Omega(\lambda),
\end{gathered}
$$

where $I=+, \dot{+}$ and $m=x, \varphi$ and we note the presence of the extra terms proportional to $Q=2$, which is a feature of the linear dilaton background. We would like to generalize the two dimensional pure spinor action (4.32) to a generic curved two dimensional non-critical background. ${ }^{8}$ Consider the matter part of the action (4.32). By using the following identity

$$
\frac{1}{2} \partial x^{m} \bar{\partial} x_{m}+p_{I} \bar{\partial} \theta^{I}=\frac{1}{2} \Pi^{m} \bar{\Pi}_{m}+\frac{Q}{2} \epsilon_{I J} \partial \theta^{I} \bar{\partial} \theta^{J}-\frac{1}{4} \tau_{I J} \theta^{I}\left(\bar{\partial} Z \partial \theta^{J}-\partial Z \bar{\partial} \theta^{J}\right)+d_{I} \bar{\partial} \theta^{I},
$$

which in ten dimensions is usually referred to as Siegel's trick [27], we can covariantize the matter part of the type II action in linear dilaton background (4.32) in the following way

$$
\begin{gathered}
S=\frac{1}{2 \pi \alpha^{\prime}} \int d^{2} z\left(\frac{1}{2} G_{M N}(Y) \partial Y^{M} \bar{\partial} Y^{N}+E_{M}^{I}(Y) d_{I} \bar{\partial} Y^{M}+E_{M}^{\hat{I}}(Y) \bar{d}_{\hat{I}} \partial Y^{M}\right) \\
-\int d^{2} z r^{(2)} \Phi(Y),
\end{gathered}
$$

where we introduced the curved supercoordinates $Y^{M}=\left(x^{m} ; \theta^{I}, \bar{\theta}^{\hat{I}}\right)$. Note that $m$ is a curved two dimensional vector index, while $I$ is a curved two dimensional spinor index. The $E_{M}^{A}$ are the zweibein superfields. We are following the notations of [14], in which the critical pure spinor action was studied in a generic ten dimensional background. In the linear dilaton case, the background superfields take the following values, the only surprise being in the metric:

\footnotetext{
${ }^{8}$ In the non-critical string it is not clear whether the concept of a background makes sense. Since the curvature is of the order of the string length, the classical supergravity approximation is not valid in general.
} 
i) The zweibeins $E_{M}^{A}$ are the two dimensional flat ones.

ii) The dilaton superfield is linear $\Phi=\frac{Q}{2}(\varphi-i x)$ and the higher components of the superfield vanish. ${ }^{9}$

iii) The metric $G_{M N}$ is constant. However in addition to the usual terms we have in flat background, in the linear dilaton background we also have a flat spinorial part

$$
G_{I J}=Q \epsilon_{I J}
$$

which is proportional to the background charge $Q=2$ and is responsible for the contribution $\epsilon_{I J} \partial \theta^{I} \partial \theta^{J}$ to the stress tensor (4.53). We regard this as a specific feature of the linear dilaton superspace structure in the pure spinor formalism. This explicitly breaks the original $S O(2)$ Lorentz invariance of the action, preserving the $U(1)_{x} \mathrm{R}$ symmetry.

It is suggestive to think of (6.24) as the matter part of the non-critical pure spinor action in a generic curved two dimensional background. It would be interesting to develop further this suggestion, in particular to work out the coupling of the pure spinor action to the curved background, in analogy to the critical case [14].

\subsubsection{Anomalies}

The pure spinor space

$$
\lambda^{+} \lambda^{\dot{+}}=0
$$

is a one-complex dimensional cone $\mathcal{M}$ with a conical singularity at $\lambda^{I}=0, I=+, \dot{+}$. The pure spinor $\lambda^{I}$ is a map from the worldsheet Riemann surface $\Sigma$ to the pure space $\mathcal{M}$. A way to eliminate the singularity is by deforming the equation to

$$
\lambda^{+} \lambda^{\dot{+}}=\mu
$$

where $\mu$ is a complex deformation parameter. The resulting (compact) space is the 2-sphere $C P^{1}$. However, since $c_{1}\left(C P^{1}\right)=2$, we cannot define the pure spinor system globally on any Riemann surface $\Sigma$ except on the 2-torus, which is unacceptable if we wish to have a complete definition of the superstring perturbation series.

Another way to eliminate the singularity is to remove the singular point. We get a disconnected space, which is the disjoint union of $C^{*}$. In this way, the anomalies are avoided. As we discussed before, a similar phenomenon occurs is the critical superstring. The difference is that while in the critical superstring case the removal of the origin still gives a connected space, here the space has two disconnected components. Note that the RNS non-critical superstring mapped to one patch of the space.

\footnotetext{
${ }^{9}$ The top form coupling to the worldsheet curvature (2.6) might be considered a component of the dilaton along the pure spinor space as well.
} 


\section{Four-dimensional superstrings}

In this section we will construct the pure spinor superstring in the four-dimensional linear dilaton background

$$
\mathbb{R}^{1,1} \times \mathbb{R}_{\varphi} \times U(1)_{x} .
$$

The four-dimensional superstring has $d+1=3$ noncompact directions $\left(x^{1}, x^{2}, \varphi\right)$ and the compact $U(1)_{x}$ direction $x$ with radius $R=2 / Q$, where $Q=\sqrt{3}$ is the Liouville background charge. The strategy will be similar to the two dimensional case.

\subsection{Multiplet spectra: RNS analysis}

In the following we will compute the spectrum of the RNS superstring in the fourdimensional linear dilaton background. We look for the short multiplets of the spacetime supersymmetry in the $\mathbb{R}^{1,1}$ directions. We follow closely the analysis of section 4.2, but postpone the details of the computation to appendix C.1. Here we briefly collect the results regarding the lowest lying operators, in particular the primaries of the worldsheet $N=2$ superconformal algebra at zero momentum in the transverse direction.

When $d=2 n$ with odd $n$, the space-time supercharges are in the same $S O(1, d-$ 1) spinor representation. In this case the two physical supercharges are

$$
\begin{aligned}
& Q_{+1}=\oint e^{-\frac{1}{2} \phi+\frac{i}{2}\left(H+H_{1}\right)-\frac{i}{2} Q x}, \\
& Q_{+2}=\oint e^{-\frac{1}{2} \phi-\frac{i}{2}\left(H-H_{1}\right)+\frac{i}{2} Q x} .
\end{aligned}
$$

They have the same $S O(1,1)_{L}$ Lorentz charge and $\mp 1 \mathrm{R}$-charge (3.8). Their OPE is

$$
Q_{+1}(z) Q_{+2}(0) \sim \frac{1}{z} e^{-\phi+i H_{1}}(0) .
$$

The other set of physical supercharges, which are nonlocal with respect to the ones above, is

$$
\begin{aligned}
& Q_{-1}=\oint e^{-\frac{1}{2} \phi+\frac{i}{2}\left(H-H_{1}\right)-\frac{i}{2} Q x}, \\
& Q_{-2}=\oint e^{-\frac{1}{2} \phi-\frac{i}{2}\left(H+H_{1}\right)+\frac{i}{2} Q x} .
\end{aligned}
$$

They have opposite $S O(1,1)$ chirality with respect to (5.1). In the type IIB superstring we GSO project both holomorphic and antiholomorphic sector with the supercharges (5.1), in the type IIA we project the antiholomorphic sector with (5.2) instead.

\subsubsection{Holomorphic sector}

NS sector 
The tachyon is non-tachyonic but is massive. Its lowest lying modes are

$$
T_{ \pm}=e^{-\phi+\frac{1}{Q}(\varphi \pm i x)}
$$

and it is a worldsheet (anti)chiral primary $\Delta_{\text {matter }}\left(T_{ \pm}\right)= \pm \frac{q}{2}=\frac{1}{2}$ annihilated by $G^{ \pm}$, with space-time R-charge $R= \pm \frac{2}{3}$. $T_{+}$and $T_{-}$are not mutually local. However, we are interested in the mutual locality only when matching holomorphic and antiholomorphic sectors, so we will discuss locality only below.

The other NS operators are analogous to the "vectors" in the ten dimensional superstring. Their lowest lying states with $p=\beta=0$ are

$$
J^{\mu}=e^{-\phi \pm H_{1}}
$$

where $\mu$ is an $S O(1,1)$ Lorentz vector index. They are worldsheet $N=2$ primaries (they have only single poles with $G^{ \pm}$) and are not charged under $U(1)_{x}$.

\section{$R$ sector}

The lowest lying components of the $\mathrm{R}$ vertex operators at zero momentum $k_{\mu}=0$ in the transverse $\mathbb{R}^{1,1}$ directions are

$$
\begin{array}{ll}
R_{++}=e^{-\frac{1}{2} \phi+\frac{i}{2}\left(H+H_{1}\right)-i \frac{Q}{2} x}, & R_{--}=e^{-\frac{1}{2} \phi-\frac{i}{2}\left(H+H_{1}\right)+\frac{i}{2 Q} x+\frac{1}{Q} \varphi}, \\
R_{+-}=e^{-\frac{1}{2} \phi+\frac{i}{2}\left(H-H_{1}\right)-\frac{i}{2 Q} x+\frac{1}{Q} \varphi}, & R_{-+}=e^{-\frac{1}{2} \phi-\frac{i}{2}\left(H-H_{1}\right)+i \frac{Q}{2} x} .
\end{array}
$$

In the following table we write the $\mathrm{R}$ charge and supersymmetry transformations of these vertex operators

$$
\begin{aligned}
& U(1)_{R} \delta_{Q_{+1}} \delta_{Q_{+2}} \\
& \begin{array}{llll}
R_{++} & -1 & 0 & J^{\mu}
\end{array} \\
& \begin{array}{llll}
R_{-+} & 1 & J^{\mu} & 0
\end{array} \\
& \begin{array}{lll}
R_{--}+1 / 3 & T_{-} & 0
\end{array} \\
& R_{+-}-1 / 3 \quad 0 \quad T_{+}
\end{aligned}
$$

In this way we can identify which supersymmetry multiplet they fall into. The last two columns list the transformations of each $\mathrm{R}$ vertex operator, obtained by applying the supercharges in (5.1).

\subsubsection{Closed superstring}

We match left and right vertex operators in IIB for concreteness, the antiholomorphic sector being a copy of the holomorphic one we just described. In type IIB we have two-dimensional $\mathcal{N}=(4,0)$ spacelike SUSY in the flat noncompact directions. Because of the requirement of mutual locality of the vertex operators, the RNS spectrum is not just the left right product of the holomorphic sector.

$N S-N S$ sector 
We have two lowest lying closed string tachyons

$$
T_{ \pm} \bar{T}_{ \pm}=e^{-\phi-\bar{\phi} \pm \frac{i}{Q}(x+\bar{x})+\frac{1}{Q}(\varphi+\bar{\varphi})}
$$

with R-charges $\pm \frac{4}{3}$. The other NS-NS operators which are primary fields of the $N=2$ are

$$
G^{\mu \nu}=e^{-\phi-\bar{\phi} \pm i H_{1} \pm i \bar{H}_{1}}
$$

which are neutral under $U(1)_{x}$.

\section{$R-R$ sector}

We match the left and right $\mathrm{R}$ states given in (5.5). By imposing the mutual locality condition we find that the lightest surviving $R-R$ fields are the six operators

$$
R_{-+} \bar{R}_{-+}, R_{-+} \bar{R}_{++}, R_{++} \bar{R}_{-+}, R_{++} \bar{R}_{++}, R_{--} \bar{R}_{--}, R_{+-} \bar{R}_{+-}
$$

They are worldsheet $N=2$ primaries as well.

\section{$R-N S$ and $N S-R$ sectors}

The fermions need not be primaries, because they are not supersymmetric deformations of the dual space-time lagrangian and only some linear combination of fermions is a definite component of a space-time short multiplet. In the following table we list the physical fermions that belong to short multiplets

$$
\begin{aligned}
& \text { NS-R }: T_{-} \bar{R}_{--}, T_{+} \bar{R}_{+-}, J^{\mu} \bar{R}_{++}, J^{\mu} \bar{R}_{-+} \\
& \text {R-NS }: R_{--} \bar{T}_{-}, R_{+-} \bar{T}_{+}, R_{++} \bar{J}^{\mu}, R_{-+} \bar{J}^{\mu}
\end{aligned}
$$

We identify which multiplet each fermion sits in by looking at its supersymmetry variation, when hit by the supercharges.

Summary: The four NS-NS operators $G^{\mu \nu}$, the first four R-R in (5.8) and the eight fermions in the last two columns of (5.9) sit in a $\mathcal{N}=(4,0) d=2$ off-shell supergravity multiplet, with a total of $16=8 \oplus 8$ states. We plot this multiplet according to its supersymmetry transformations as follows

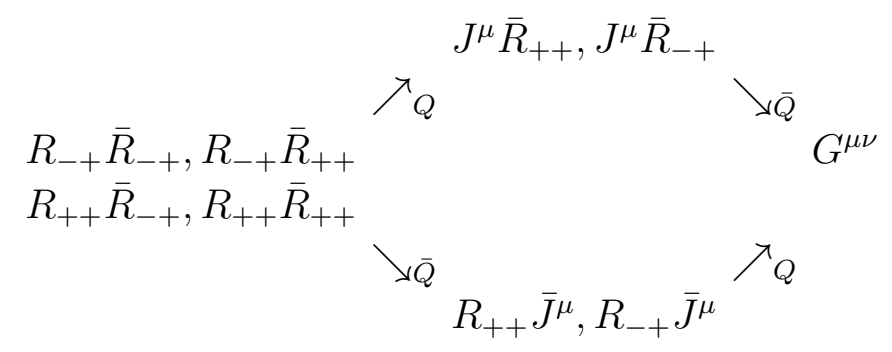


where $Q$ and $\bar{Q}$ represent the holomorphic and antiholomorphic supercharges. We then find a gauge supermultiplet with $4 \oplus 4$ degrees of freedom, obtained as a combination of two chiral multiplets, whose top components are the two tachyons. One multiplet is

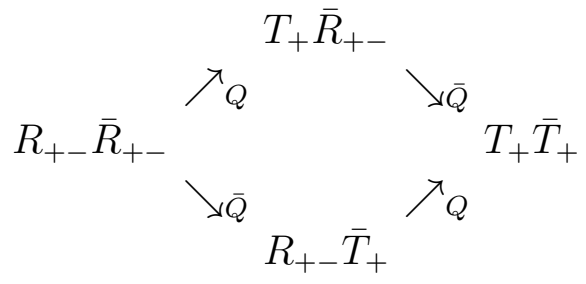

and analogously for the other one $T_{-} \bar{T}_{-}, R_{--} \bar{T}_{-}, T_{-} \bar{R}_{--}, R_{--} \bar{R}_{--}$.

Collecting the results, we find the physical spectrum of $12 \oplus 12$ operators in (3.10).

\subsection{Pure spinor variables}

The RNS superstring in the four dimensional linear dilaton background has two supercharges both in the right moving and in the left moving sectors. Let us focus on the holomorphic sector only. The closed superstring in this formalism will just be the left right product of the two sectors, without the complications of the mutual locality conditions we found in the RNS. We follow the same strategy discussed in section 4.3 for the two dimensional linear dilaton, but here we will introduce an additional ingredient, the flat $\mathbb{R}^{1,1}$ part of the space-time.

The physical RNS supercharges $Q_{+1}, Q_{+2}$ are given in (5.1). As noted in [4], there exist other two additional supercharges

$$
\begin{aligned}
& Q_{\dot{+} 1}=\oint e^{-\frac{1}{2} \phi-\frac{i}{2}\left(H_{1}-H\right)+\frac{i}{2} Q x}, \\
& Q_{\dot{+} 2}=\oint e^{-\frac{1}{2} \phi-\frac{i}{2}\left(H_{1}+H\right)-\frac{i}{2} Q x}
\end{aligned}
$$

which survive GSO projection, are mutually local with respect to the physical ones (5.1) and are conserved, i.e. they have at most double poles with the stress tensor. However, they are not in the BRST cohomology, as they do not correspond to any physical space-time symmetry. They play a key role in the construction of the covariant formalism.

As we will see, the RNS variables will be mapped into a patch of the pure spinor space. The pure spinor degrees of freedom of the four-dimensional superstring are an $S O(1,3)$ Dirac spinor $\lambda^{A}, A=1, \ldots, 4$ satisfying the conditions

$$
\lambda \Gamma^{m} \lambda=0
$$

where $\Gamma^{m}$ are the $4 \times 4$ four-dimensional Dirac matrices. To perform the map it is most convenient to solve the pure spinor constraint by breaking $S O(1,3)$ to $U(2)$ and decompose the Dirac spinor as a $\left(\lambda, \lambda^{a}, \lambda_{a b}\right)$, namely a singlet, a two component 
vector and a one component antisymmetric irreducible representations, for the details see the Appendix. The pure spinor conditions become

$$
\lambda \lambda^{a}=0, \quad \lambda_{a b} \lambda^{a}=0 .
$$

They can be solved by going to the patch where $\lambda^{a}=0$, so we are left with $\left(\lambda, \lambda_{a b}\right){ }^{10}$

Let us consider the four RNS supercharges, both physical (5.1) and unphysical ones (5.10). We take $Q_{+2}$ in the $+\frac{1}{2}$ picture and all the others in the $-\frac{1}{2}$ picture and we recast them in the a $U(2)$ notation: $Q_{+1} \equiv Q_{+},\left(Q_{+2}, Q_{\dot{+} 1}\right) \equiv Q_{a}, Q_{\dot{+} 2} \equiv Q^{a b}$. We define

$$
\begin{array}{r}
\theta^{+}=c \xi e^{-\frac{3}{2} \phi-\frac{i}{2} H_{1}-\frac{i}{2}(H-Q x)}, p_{+}=b \eta e^{\frac{3}{2} \phi+\frac{i}{2} H_{1}+\frac{i}{2}(H-Q x)} \\
\theta^{a}=e^{\frac{1}{2} \phi \mp \frac{i}{2}\left(H_{1}-H\right)-\frac{i}{2} Q x}, p_{a}=e^{-\frac{1}{2} \phi \pm \frac{i}{2}\left(H_{1}-H\right)+\frac{i}{2} Q x} \\
\theta_{a b}=e^{\frac{1}{2} \phi+\frac{i}{2} H_{1}+\frac{i}{2}(H+Q x)}, p^{a b}=e^{\frac{-1}{2} \phi-\frac{i}{2} H_{1}-\frac{i}{2}(H+Q x)}
\end{array}
$$

where the $\theta$ 's are the conjugate variables to the $p$ 's. The OPE of the first three conjugate pairs correspond to free fields

$$
p_{+}(z) \theta^{+}(w) \sim \frac{1}{(z-w)}, \quad p_{a}(z) \theta^{b}(w) \sim \frac{\delta_{a}^{b}}{(z-w)},
$$

where $a, b=1,2$ and all the others vanishing. However, the OPE of $p^{a b}$ and $\theta_{a b}$ have poles with all the others, so we drop these two components. We will recover them later as a BRST quartet. ${ }^{11}$ The way to map the bosonized RNS variables to the pure spinors is to use the $U(2)$ singlet $p_{+}$in the $+\frac{1}{2}$ picture and set

$$
\eta=p_{+} e^{\tilde{\phi}+\tilde{\kappa}}, \quad b=p_{+} e^{\frac{1}{2}(\tilde{\phi}-\tilde{\kappa})} .
$$

The bosons $\tilde{\phi}$ and $\tilde{\kappa}$ satisfy the OPE's (4.20). We redefine $x \rightarrow x^{\prime}$ to have the correct free field OPE's among our new variables

$$
x^{\prime}=\frac{1}{2}\left(x-Q\left(H_{1}+H-i \phi\right)\right) .
$$

The total RNS stress tensor is mapped to

$$
\begin{aligned}
T_{m}+T_{g h}= & -\frac{1}{2} \sum_{\mu=1}^{2}\left(\partial x^{\mu}\right)^{2}-\frac{1}{2}\left(\partial x^{\prime}\right)^{2}-\frac{1}{2}(\partial \varphi)^{2}+\frac{Q}{2} \partial^{2}\left(\varphi-i x^{\prime}\right) \\
& -p_{+} \partial \theta^{+}-p_{a} \partial \theta^{a}-\frac{1}{2}(\partial \tilde{\phi})^{2}+\partial^{2} \tilde{\phi}+\frac{1}{2}(\partial \tilde{\kappa})^{2}+\partial^{2} \tilde{\kappa}
\end{aligned}
$$

\footnotetext{
${ }^{10}$ Another way to write the patch is to parameterize the pure spinor degrees of freedom as the Weyl and anti-Weyl spinors $\left(\lambda^{\alpha}, \lambda^{\dot{\alpha}}\right)$ with the pure spinor constraints $\lambda^{\alpha} \sigma_{\alpha \dot{\beta}}^{m} \lambda^{\dot{\beta}}=0$. Since $\sigma^{m}$ is a complete basis in the space of two-dimensional bispinors, the pure spinor condition becomes simply $\lambda^{\alpha} \lambda^{\dot{\alpha}}=0$. In fact, we can identify the two parametrizations by noting that $\lambda^{\alpha}=\lambda^{a}, \lambda^{\dot{\alpha}}=\left(\lambda, \lambda_{a b}\right)$, so the patch $\lambda^{a}=0$ reads $\lambda^{\alpha}=0$. In the following we will map the RNS theory to this patch.

${ }^{11}$ Note that while $\left(\theta^{+}, p_{+}\right)$and $\left(\theta^{1}, p_{1}\right)$ are BRST invariant, the remaining $\left(\theta^{2}, p_{2}\right)$ are used in order to enlarge the superspace structure but are not physical. We will recover the appropriate physical superspace when discussing the pure spinor global symmetries.
} 
The second step is to use the $\tilde{\phi}, \tilde{\kappa}$ as the ordinary bosonization of a $\beta, \gamma$ system of weight $(1,0)$ as in 4.26$)$, representing now the $U(2)$ singlet components of the pure spinor and its conjugate momentum as in (5.12).

If we take a closer look at the stress tensor for the bosonized pure spinors $\tilde{\phi}, \tilde{\kappa}$ we see that the same story as in two dimensions is repeated. There is a mismatch between the stress tensor we get from the map from the RNS and the naive stress tensor $w_{+} \partial \lambda^{+}$one would expect for the free beta-gamma system. This is the contribution coming from the coupling of the top form on the pure spinor manifold $\Omega(\lambda)$ to the worldsheet curvature (2.6). We find again that the pure spinor stress tensor we get from the map is (4.29) and the top form is (4.30). Due to the coupling (2.6) of the top form to the action, we need three powers of $\lambda^{+}$in the saturation rule to get a nonvanishing amplitude.

The total central charge of the system still vanishes

$$
c=(-2)_{p_{+} \theta^{+}}+(-4)_{p_{a} \theta^{a}}+(2)_{x_{1}, x_{2}}+(1+12)_{\varphi}+(1-12)_{x^{\prime}}+(2)_{w \lambda}=0 .
$$

In four dimensions there is a new feature with respect to the two-dimensional case. We need to add a topological quartet with central charge $c=0$ to reconstruct the target space structure on the pure spinor side. We add a fermionic $b c$ system of weight one $\left(p^{a b}, \theta_{a b}\right)$ and a bosonic $\beta \gamma$ system of weight one with the same quantum numbers $\left(w^{a b}, \lambda_{a b}\right)$.

We suitably modify the currents of the twisted $N=2$ by adding a term that depends on the quartet. In this way we can recast the stress tensor (5.19) in the following covariant way

$$
\begin{aligned}
T= & -\frac{1}{2}\left(\partial x^{\mu}\right)^{2}-\frac{1}{2}\left(\partial x^{\prime}\right)^{2}-\frac{1}{2}(\partial \varphi)^{2}+\frac{Q}{2} \partial^{2}\left(\varphi-i x^{\prime}\right)-p_{I i} \partial \theta^{I i} \\
& +w_{I i} \partial \lambda^{I i}-\frac{1}{2} \partial^{2} \log \Omega(\lambda) .
\end{aligned}
$$

The index $I=+, \dot{+}$, where $I=+$ corresponds to the physical superspace coordinates and conjugate momenta and $I=\dot{+}$ represents the variables of the enlarged superspace. The index $I$ keeps track of the $S O(1,1)$ Lorentz spinor chirality, namely $I=+$ is $+\frac{1}{2}$ Lorentz charge and $I=\dot{+}$ is $-\frac{1}{2}$; the index $i=1,2$ labels two different spinors with the same chiralities. Note that we do not have any spinor index in the game since all the spinors are in the Majorana Weyl representation. Moreover, we reconstructed the full pure spinor $\lambda^{I i}$. The matter part of this stress tensor can be derived from the pure spinor action

$$
\begin{array}{r}
S=\frac{1}{2 \pi \alpha^{\prime}} \int d^{2} z\left(\frac{1}{2} \partial x^{\mu} \bar{\partial} x^{\nu} \eta_{\mu \nu}+\frac{1}{2} \partial x \bar{\partial} x+\frac{1}{2} \partial \varphi \bar{\partial} \varphi+p_{I i} \bar{\partial} \theta^{I i}\right) \\
-\frac{Q}{2} \int d^{2} z r^{(2)}(\varphi-i x)
\end{array}
$$


where the last term is the Fradkin-Tseytlin term that couples the space-time linear dilaton $\Phi=-\frac{Q}{2}(\varphi-i x)$ to the worldsheet curvature (we denoted $x^{\prime}$ by $x$ ). For the consistency of the FT term we have to compactify the $x$ direction on a circle of radius $R=2 / Q$, which in fact is the supersymmetric radius we already know from RNS analysis.

Mapping the RNS saturation rule on the sphere to the pure spinor variables one gets a requirement for an insertion of $\lambda_{+}^{3}$, which is consistent with the measure corresponding to the top form we obtained. Finally, the ghost current on the patch ${ }^{12}$ $J_{g h}=-\partial \tilde{\phi}+w^{a b} \lambda_{a b}$ can be written coviariantly as

$$
J_{g h}=w_{I i} \lambda^{I i} .
$$

\subsubsection{Supersymmetry structure}

When constructing the map from the RNS to the pure spinors, we doubled the superspace. Namely, we supplement the physical supercharges $Q_{+1}, Q_{+2}$ with two additional supercharges $Q_{\dot{+} 1}, Q_{\dot{+} 2}$ that are not BRST invariant, thus not physical, although they are conserved. We proceed as in the two dimensional case by looking at the algebra we get from the RNS. Since the RNS supersymmetry algebra closes up to picture changing, we take the supercharge $Q_{+2}$ in (5.1) in the $+\frac{1}{2}$ picture

$$
\begin{gathered}
q_{+2}=b \eta e^{\frac{3}{2} \phi+\frac{i}{2} H_{1}+\frac{i}{2}(H-Q x)}+\partial\left(x_{1}+i x_{2}\right) e^{\frac{1}{2} \phi-\frac{i}{2} H_{1}+\frac{i}{2} H-\frac{i}{2} Q x}+ \\
+\partial(\varphi+i x+i Q H) e^{\frac{1}{2} \phi+\frac{i}{2} H_{1}-\frac{i}{2} H-\frac{i}{2} Q x}
\end{gathered}
$$

and the other three in the $-\frac{1}{2}$ picture. The OPE's between them are

$$
\begin{aligned}
& q_{+1}(z) q_{+2}(0) \sim \frac{1}{z} \partial\left(x_{1}+i x_{2}\right)(0), \\
& q_{+2}(z) q_{\dot{+} 1}(0) \sim \frac{Q}{z^{2}}+\frac{1}{z} \partial(\varphi+i x+i Q H)(0)
\end{aligned}
$$

and $q_{+2}(z) q_{\dot{+} 2} \sim r e g$. If we map this algebra to the pure spinor variables we find that the first equation remains unchanged, while the second reads

$$
q_{+2}(z) q_{+1}(0) \sim \frac{Q}{z^{2}}+\frac{1}{z} \partial(\varphi-i x)(0),
$$

where we denoted $x^{\prime}$ by $x$ for simplicity of notation. As usual, the algebra is realized on the superderivatives with opposite signs.

With these variables we construct the following GS-like constraints

$$
d_{I j}=p_{I j}-\frac{1}{2} \tau_{i j}\left(\delta_{I J} \theta^{J i} \partial\left(x_{1}+i x_{2}\right)+\tau_{I J} \theta^{J i} \partial(\varphi-i x)-Q \epsilon_{I J} \partial \theta^{J i}\right),
$$

where $\tau$ is the $\sigma^{1}$ Pauli matrix and $\epsilon_{I J}$ is the antisymmetric matrix. The GS-like constraints are similar to the $d=0$ case (4.36), except for the new term proportional

\footnotetext{
${ }^{12}$ We obtain this current by mapping the RNS ghost current, adding to it the contribution of the quartet and adding the term $-\frac{3}{2}(\partial \tilde{\phi}-\partial \tilde{\kappa})$, which does not alter the anomalies nor the ghost number but might be useful for the Lorentz properties.
} 
to $\delta_{I J}$, which realizes the $d=2$ space-time supersymmetry. The notations are explained in (5.21). These constraints reproduce the algebra we mapped from the RNS superderivatives (given by the supercurrent algebra (5.26) but with the opposite signs)

$$
d_{I i}(z) d_{J j}(0) \sim-\tau_{i j} \frac{\epsilon_{I J} Q}{z^{2}}-\tau_{i j} \frac{\delta_{I J}}{z} \partial\left(x_{1}+i x_{2}\right)(0)-\tau_{i j} \frac{\tau_{I J}}{z} \partial(\varphi-i x)(0) .
$$

Let us briefly discuss this algebra. The second term tells us that the physical $d_{+1}$, $d_{+2}$ close on the space-time $S O(1,1)$ holomorphic translation generator, exactly reproducing the RNS supersymmetry. The two unphysical superderivatives $d_{\dot{+} 1}, d_{\dot{+} 2}$ close on the same translation generator. However, the two physical and unphysical superderivatives have a double pole in their OPE, proportional to the Liouville background charge $Q$. Therefore the non anomalous worldsheet current algebra realizes $\mathcal{N}=(2,0)$ supersymmetry in two dimensions. In the closed type IIB theory we get just the left right product of the two sectors, realizing $\mathcal{N}=(4,0)$ two dimensional supersymmetry, as we expect from the RNS analysis.

Let us recall the space-time symmetries. In each of the two sectors, the expectation value of the dilaton and the compactification of $x$ on a circle break the four dimensional Lorentz symmetry with four supercharges to two real supersymmetries and a bosonic $S O(1,1) \times\left(U(1) \times \mathbb{Z}_{2}\right)_{R}$. Under the bosonic $S O(1,1) \times U(1)_{x}$ symmetry the $d$ 's are charged according to

$$
\begin{array}{ccc} 
& S O(1,1) & U(1)_{x} \\
d_{+1} & +\frac{1}{2} & +1 \\
d_{+2} & +\frac{1}{2} & -1 \\
d_{\dot{+} 1} & -\frac{1}{2} & +1 \\
d_{\dot{+} 2} & -\frac{1}{2} & -1
\end{array}
$$

The physical worldsheet holomorphic current algebra realizes space-time $\mathcal{N}=(2,0)$ supersymmetry in two dimensions

$$
d_{+1}(z) d_{+2}(0) \sim-\frac{\partial\left(x_{1}+i x_{2}\right)(0)}{z} .
$$

When acting on superfields depending on the field zero modes only, the $d_{I i}$ looks like a superderivative

$$
d_{I i}(z) \Phi\left(\bar{Z}, x_{1}, x_{2}, \theta^{I i}\right)(0) \sim-\frac{1}{z} D_{I i} \Phi\left(\bar{Z}, x_{1}, x_{2}, \theta^{I i}\right)(0)
$$

where

$$
D_{I i}=\partial_{\theta^{I i}}+\tau_{i j}\left(\delta_{I J} \partial_{x_{1}-i x_{2}}+\tau_{I J} \partial_{Z}\right)
$$

and the notations is the same as in the two dimensional case $Z=\varphi+i x, \bar{Z}=\varphi-i x$. 


\subsubsection{Cohomology}

The pure spinor BRST charge in the four-dimensional non-critical string is

$$
Q_{B}=\oint \lambda^{I i} d_{I i}
$$

where the GS-like constraints $d_{I i}$ in the linear dilaton background are defined in (5.27). As discussed in section 4.3.2, we included all the physical as well as the unphysical d's in the BRST charge. We will compute the cohomology in two steps. First we compute the cohomology of $Q_{B}$ in the enlarged superspace containing all the $\theta^{I i}$, then we restrict to the part of the cohomology that contains the variables that realize the supersymmetry current algebra, namely the $\theta^{+i}$.

Let us discuss the nilpotency of the BRST charge $Q_{B}$. The four-dimensional pure spinor constraint (5.11) can be recast according to the notation of the linear dilaton background in the form ${ }^{13}$

$$
\tau_{i j} \lambda^{I i} \lambda^{J j}=0
$$

for $I, J=+\dot{+}$ and $i, j=1,2$. Due to the OPE (5.28), the nilpotency of the BRST charge requires the following conditions

$$
\tau_{i j} \delta_{I J} \lambda^{I i} \lambda^{J j}=0, \quad \tau_{i j} \tau_{I J} \lambda^{I i} \lambda^{J j}=0
$$

which are implied by the pure spinor condition (5.34). Due to the double pole in (5.28), however, we have an additional derivative constraint

$$
\epsilon_{I J} \tau_{i j} \partial \lambda^{I i} \lambda^{J j}=0
$$

One can show that the pure spinor condition (5.34) implies that both $\tau_{i j} \lambda^{+i} \partial \lambda^{+j}$ and $\tau_{i j} \partial \lambda^{+i} \lambda^{+j}$ vanish separately. The way to prove it is analogous to the $d=0$ case we discussed in (4.39), by looking at the mode expansion of the pure spinor constraint (5.34) or alternatively by analyzing the OPE's involving the Lorentz generator and the ghost current [26, 28].

Let us compute the cohomology now. As discussed in the two-dimensional case, there are two different kinds of vertex operators at ghost number one and weight zero. The first one is

$$
\mathcal{V}^{(1)}=\lambda^{I i} A_{I i}\left(\bar{Z}, x^{\mu}, \theta^{I i}\right)
$$

Whereas in the previous case (4.40) we found that this operator was exact (there is no gravity in $d=0$ ), now we will find an off-shell two dimensional supergravity

13 The constraint $\lambda^{A} \Gamma_{A B}^{M} \lambda^{B}=0$ can be written in flat Weyl notation as $\lambda^{\alpha} \lambda^{\alpha}=0$ where $\lambda^{A}=\left(\lambda^{\alpha}, \lambda^{\dot{\alpha}}\right)$. Then we identify the flat Weyl indices with our linear dilaton quantum numbers as I $i=+1 \rightarrow \alpha=1, I i=\dot{+} 1 \rightarrow \alpha=2, I i=+2 \rightarrow \dot{\alpha}=\dot{1}$ and $I i=\dot{+} 2 \rightarrow \dot{\alpha}=\dot{2}$. 
multiplet. Imposing that $\mathcal{V}$ is BRST closed on the pure spinor condition (5.34), we get

$$
D_{(I 1} A_{J 1)}=0, \quad D_{(I 2} A_{J 2)}=0,
$$

where $D_{I i}$ is defined in (5.32). Due to the algebra

$$
\left\{D_{I 1}, D_{J 1}\right\}=0=\left\{D_{I 2}, D_{J 2}\right\}
$$

we can solve these two equations by choosing $A_{I 1}=D_{I 1} B$ and $A_{I 2}=D_{I 2} C$, for two generic superfields $B$ and $C$. Let us take equivalently a linear combination $B=M+N$ and $C=M-N$, so that $A_{I 1}=D_{I 1}(M+N)$ and $A_{I 2}=D_{I 2}(M-N)$. Now we require that this vertex operator is not BRST exact, that is we mod out by the following gauge invariance

$$
\delta \mathcal{V}^{(1)}=Q_{B} \Omega^{(0)}
$$

where $\Omega^{(0)}\left(\bar{Z}, x^{\mu}, \theta^{I i}\right)$ is a generic ghost number zero and weight zero superfield. If we choose $\Omega=-M$, we are left with

$$
A_{I 1}=D_{I 1} N, \quad A_{I 2}=-D_{I 2} N
$$

for a generic superfield $N$. It is easy to see that the degrees of freedom encoded in $\mathcal{V}$ are $4 \oplus 4$, which is the result of $[29,26] .{ }^{14}$ Since we are interested in the $\operatorname{SO}(1,1)$ supersymmetry multiplet, we have to eliminate the $\theta^{+i}$ components in the vertex operator, keeping only the $\theta^{+i}$, the latter entering in the holomorphic space-time $\mathcal{N}=(2,0)$ current algebra. The physical states in the holomorphic sector consist finally of $2 \oplus 2$ degrees of freedom of an off-shell $S O(1,1)$ vector supermultiplet.

We are interested in the closed string spectrum, which is the tensor product of the holomorphic and antiholomophic sectors. The cohomology computation of the closed string vertex operator

$$
\mathcal{V}^{(1,1)}=\lambda^{I i} \bar{\lambda}^{J j} A_{I i, J j}\left(\bar{Z}, x^{\mu}, \theta^{I i}, \bar{\theta}^{K k}\right),
$$

where the $\bar{\lambda}$ and $\bar{\theta}$ are the antiholomorphic variables, gives a total of $8 \oplus 8$ degrees of freedom, that fit into an $\mathcal{N}=(4,0) d=2$ supergravity multiplet, reproducing the RNS computation.

The second type of vertex operator is the generalization of the two-dimensional one that we introduced in (4.47). This accounts for the gauge multiplet, which is the other character in the cohomology of the linear dilaton superstring. In this case the computation of the cohomology is more tedious, and we present the details in

\footnotetext{
${ }^{14}$ This is done most quickly in the notations in the previous footnote, by which $A_{I 1} \equiv A_{\alpha}$ and $A_{I 2} \equiv A_{\dot{\alpha}}$. If we introduce the supercurvature $F_{A B}=D_{(A} A_{B}$, for $A, B=(\alpha, \dot{\alpha})$ then (5.41) is equivalent to the following conditions on the curvature $F_{\alpha \beta}=F_{\dot{\alpha} \dot{\beta}}=F_{\alpha \dot{\beta}}=0$, which are the usual superspace constraints defining the $\mathcal{N}=1$ off-shell vector multiplet in four dimensions.
} 
appendix D. After taking into account the equations of motion modulo the gauge symmetries and projecting to the physical supercoordinates $\theta^{+i}$, the vertex operator for this gauge supermultiplet in the holomorphic sector is

$$
\mathcal{U}_{T}^{(1)}=\lambda^{I j} \partial \theta^{+i} D_{I j} T_{i}\left(\theta^{+i}\right)
$$

where the wavefunctions of the superfields $T_{i}$ are given by $e^{-Z / Q}$, by which we get a weight zero vertex operator. To compare this vertex operator to the RNS, we need to take into account the structure of the pure spinor space. The four-dimensional pure spinor space $\tau_{i j} \lambda^{I i} \lambda^{J j}=0$ is the union of two disconnected patches and our vertex operator (5.43) is defined globally on the two patches exchanged by $\lambda^{I 1} \leftrightarrow \lambda^{I 2}$. The RNS formalism is mapped onto just one of the two patches, let us choose the $\lambda^{I 2} \neq 0$ patch. In order to compare the RNS cohomology with the pure spinor result, we will consider one of the two disconnected patches, so the tachyon supermultiplet on the patch $\lambda^{I 2} \neq 0$ has the form

$$
\mathcal{U}^{(1)}=\lambda^{I 2} \partial \theta^{1} D_{I 2} T_{1}\left(\theta^{1}, \theta^{\mathrm{i}}\right)
$$

containing $2 \oplus 2$ degrees of freedom. In the closed string, we have to take the product of the holomorphic and antiholomorphic vertex operators. Since we are comparing the pure spinor computation with the RNS cohomology at zero momentum, we need to mod out by the center of the space-time symmetries $S O(1,1) \times U(1)_{x} \times \mathbb{Z}_{2}$, which is just the $\mathbb{Z}_{2}$, so that we recover the closed superstring $4 \oplus 4$ supermultiplet of (3.10).

Let us make a few comments. In the two-dimensional case, the gauge supermultiplet $(4.50)$ was the only physical multiplet present in the second type vertex operator at ghost number one and weight zero. This is in agreement with the known fact that in the two-dimensional non-critical superstrings in the linear dilaton background the tower of "massive" string states is absent. In the present four-dimensional case, however, this second type of vertex operator contains other physical states in addition to the gauge supermultiplet which we just described. These additional states are some of the tower of higher states in the cohomology of the superstring, which we expect to be present. ${ }^{15}$ The study of the higher states in the superstring cohomology might be interesting by itself.

\subsubsection{Curved non-critical backgrounds}

We follow the discussion in section 4.3 .3 and suggest a generalization of the noncritical pure spinors to generic four dimensional curved backgrounds with at most eight real supercharges.

\footnotetext{
${ }^{15}$ We would not call these higher states massive, since, as we explained in section 3 , in the linear dilaton background the space-time supermultiplets are off-shell, in the sense that we do not have any dispersion relation.
} 
Even if the linear dilaton has only flat two dimensional supersymmetry, we introduce now the momenta $\Pi^{m}$, for $m=1,2, \varphi, x$, that will be useful when casting the theory in a background with twice as many supersymmetries

$$
\begin{array}{cc}
\Pi^{1}=\partial x^{1}+\frac{1}{2} \delta_{I J} \tau_{i j} \theta^{I i} \partial \theta^{J j}, & \Pi^{2}=\partial x^{2}+\frac{i}{2} \delta_{I J} \tau_{i j} \theta^{I i} \partial \theta^{J j} \\
\Pi^{\varphi}=\partial \varphi+\frac{1}{2} \tau_{I J} \tau_{i j} \theta^{I i} \partial \theta^{J j}, & \Pi^{x}=\partial x-\frac{i}{2} \tau_{I J} \tau_{i j} \theta^{I i} \partial \theta^{J j}
\end{array}
$$

They satisfy the following algebra

$$
\begin{array}{cc}
\Pi^{n}(z) \Pi^{m}(0) \sim-\frac{\eta^{m n}}{z^{2}} \\
d_{I i}(z) \Pi^{1}(0) \sim \frac{\delta_{I J} \tau_{i j}}{z} \partial \theta^{J j}(0), & d_{I i}(z) \Pi^{2}(0) \sim \frac{i \delta_{I J} \tau_{i j}}{z} \partial \theta^{J j}(0) \\
d_{I i}(z) \Pi^{\varphi}(0) \sim \frac{\tau_{I J} \tau_{i j}}{z} \partial \theta^{J j}(0), & d_{I i}(z) \Pi^{x}(0) \sim \frac{i \tau_{I J} \tau_{i j}}{z} \partial \theta^{J j}(0)
\end{array}
$$

The stress tensor (5.21) can be cast in the form

$$
\begin{aligned}
T=-\frac{1}{2} \Pi^{m} \Pi^{n} \eta_{m n}- & d_{I i} \partial \theta^{I i}+\frac{Q}{2} \epsilon_{I J} \tau_{i j} \partial \theta^{I i} \partial \theta^{J j}+\frac{Q}{2} \partial\left(\Pi^{\phi}-i \Pi^{x}\right) \\
& +w_{I i} \partial \lambda^{I i}-\frac{1}{2} \partial^{2} \log \Omega(\lambda)
\end{aligned}
$$

Note the presence of the extra terms proportional to $Q=\sqrt{3}$, which is a feature of the linear dilaton background. Similarly, we can write the action in these variables, which correspond to the supervielbeins of the general four-dimensional pure spinor superstring backgrounds

$$
\begin{gathered}
S=\frac{1}{2 \pi \alpha^{\prime}} \int d^{2} z\left(\frac{1}{2} G_{M N}(Y) \partial Y^{M} \bar{\partial} Y^{N}+E_{M}^{A}(Y) d_{A} \bar{\partial} Y^{M}+E_{M}^{A}(Y) \bar{d}_{A} \partial Y^{M}\right) \\
-\int d^{2} z r^{(2)} \Phi(Y)
\end{gathered}
$$

where we introduced the curved supercoordinates $Y^{M}=\left(x^{m} ; \theta^{A}, \bar{\theta}^{\hat{A}}\right)$ and $m$ is a curved four dimensional vector index, while $A=(\alpha, \dot{\alpha})$ is a curved four dimensional Dirac spinor index; the relation between $A$ and $I i$ is explained in the appendix. The variables without bars are in the holomorphic sector, the ones with bars are in the antiholomorphic sector. The E's are the vierbein superfields. The whole story is a generalization of the two dimensional case. In the linear dilaton case, the background superfields take the following values. The vierbeins $E$ 's are the four dimensional flat ones. The dilaton superfield is linear $\Phi=\frac{Q}{2}(\varphi-i x)$. The metric $G_{M N}$ is constant, however in addition to the usual terms appropriate for a flat background, we have a flat spinorial part as well

$$
G_{\alpha \dot{\alpha}}=i Q \sigma_{\alpha \dot{\alpha}}^{2}, \quad G_{\hat{\alpha} \hat{\alpha}}=i Q \sigma_{\hat{\alpha} \hat{\alpha}}^{2}
$$


which is proportional to the background charge $Q=\sqrt{3}$ and is responsible for the contribution $Q \epsilon_{I J} \tau_{i j} \partial \theta^{I i} \partial \theta^{J j}$ to the stress tensor (4.53). We regard this as a specific feature of the linear dilaton superspace structure in the pure spinor formalism. This explicitly breaks the parent $S O(1,3)$ Lorentz invariance of the action down to $S O(2)$, preserving the $U(1)_{x} \mathrm{R}$ symmetry.

It is suggestive to think of (5.49) as the matter part of the non-critical pure spinor action in a generic curved four dimensional background. We will discuss the non-critical $A d S_{4}$ example in section 8.

\subsubsection{Anomalies}

The pure spinor degrees of freedom in the Weyl notations are $\left(\lambda^{\alpha}, \lambda^{\dot{\alpha}}\right)$ with the conditions

$$
\lambda^{\alpha} \lambda^{\dot{\alpha}}=0
$$

These complexified equations define a two-complex dimensional space with a conical singularity at $\lambda^{\alpha}=\lambda^{\dot{\alpha}}=0$.

As in the two-dimensional space, deforming this set of four complex equations results in a space whose first Chern class is nonvanishing, leading to an anomalous theory. Again, we can eliminate the singularity by removing the singular point. We get a disconnected space, which is the disjoint union of $C^{*} \times C^{*}$ s. In this way, the anomalies are avoided and as in the two-dimensional case the resulting space is disconnected.

\section{Six-dimensional superstrings}

In this section we discuss the six dimensional non-critical superstring in the linear dilaton background

$$
\mathbb{R}^{1,3} \times \mathbb{R}_{\varphi} \times U(1)_{x}
$$

We will closely follow the analysis in the previous Sections on $d=0$ and $d=2$, so we will skip some details. The flat $\mathbb{R}^{1,3}$ coordinates are $x^{\mu}$, while $x$ is compactified on a circle of radius $2 / Q$, the Liouville background charge is $Q=\sqrt{2}$ and we have $d=2 n=4$.

\subsection{Multiplet spectra: RNS analysis}

The space-time supercharges have different chiralities of the Lorentz group $S O(1,3)$. There are sixteen candidates for the supercharges. They form two groups of eight mutually local physical operators. One such group is given by (in the $-\frac{1}{2}$ picture)

$$
\begin{array}{ll}
q_{+1}=e^{-\frac{1}{2} \phi+i\left(H^{1}-H^{2}-H+Q x\right) / 2}, & q_{+2}=e^{-\frac{1}{2} \phi+i\left(-H^{1}+H^{2}-H+Q x\right) / 2}, \\
q_{+\dot{1}}=e^{-\frac{1}{2} \phi+i\left(H^{1}+H^{2}+H-Q x\right) / 2}, & q_{+\dot{2}}=e^{-\frac{1}{2} \phi+i\left(-H^{1}-H^{2}+H-Q x\right) / 2},
\end{array}
$$


Defining $\sigma_{1}=\mathbf{1}, \sigma_{2}=\tau_{1}, \sigma_{3}=i \tau_{3}$ and $\sigma_{4}=\tau_{2}$, where the $\tau$ s are the Pauli matrices, we can rewrite the above OPE's in the concise form

$$
q_{+\alpha}(z) q_{+\dot{\alpha}}(0) \sim \frac{1}{\sqrt{2} z} \sigma_{i \alpha \dot{\alpha}} e^{-\phi} \psi^{i}(0)
$$

The other set of supercharges $q_{-\alpha}, q_{-\dot{\alpha}}$, which are physical but nonlocal with respect to (6.1) is listed in the Appendix. We will choose the $q_{+}$set in the holomorphic sector. The choice of the $\bar{q}_{+}$set in the antiholomorphic sector as well defines type IIB superstring. The other choice of the $\bar{q}_{-}$set in the antiholomorphic sector defines the type IIA superstring. In both cases we realize $\mathcal{N}=2$ space-time supersymmetry in the flat $S O(1,3)$ directions. In the following we will stick to the type IIB case.

Let us collect the short space-time supermultiplets of the type IIB superstring. The details of the computations are listed in the Appendix.

\subsubsection{Holomorphic sector}

The holomorphic sector is obtained by requiring mutual locality with the supercharges $q_{+\alpha}$ and $q_{+\dot{\alpha}}$. This will serve as a building block for the closed superstring states we will consider in the next section.

The first NS state at zero transverse momentum is the tachyon whose two lowest lying states are

$$
T^{ \pm}=\varphi e^{-\phi+\frac{1}{Q}(\varphi \pm i x)},
$$

which carry R-charges \pm 1 . The factor of $\varphi$ in front of the vertex operator comes from the requirement of non-normalizability and is specific to $d+2=6$.

At the next level there are the NS vectors. The zero momentum states are

$$
J_{\mu}=e^{-\phi \pm i H^{I}}
$$

We now turn to the Ramond sector. We use Polchinski's notation $(\alpha, F)$ [30], where $\alpha$ is the space-time fermion index and $F$ is the worldsheet spinor index, and denote the $\mathrm{R}$ operators by $R^{F}$. The zero momentum $\mathrm{R}$ states with $F=1$ are

$$
\begin{array}{ll}
R_{+++}^{1}=e^{-\phi / 2+i\left(H+H^{1}+H^{2}\right) / 2-i x / Q}, & R_{--+}^{1}=e^{-\phi / 2+i\left(-H+H^{1}-H^{2}\right) / 2+i x / Q}, \\
R_{-+-}^{1}=e^{-\phi / 2-i\left(H+H^{1}-H^{2}\right) / 2-i x / Q}, & R_{+--}^{1}=e^{-\phi / 2-i\left(-H+H^{1}+H^{2}\right) / 2+i x / Q}
\end{array}
$$

that are the four physical supercharges (6.1). ${ }^{16}$ According to the supersymmetry algebra (6.2), they are mapped to the four NS states in (6.4), schematically $\delta_{\text {susy }} R^{1}=$

\footnotetext{
${ }^{16}$ In terms of a full $S O(6)$ spinor representation, these $\mathrm{R}$ states have an odd number of +'s and correspond to a $\underline{4} \in S U(4) \simeq S O(6)$.
} 
$J$. The $F=0$ states with zero momentum and zero R-charge have $\beta=\frac{Q}{2}$ so we have to pick the non-normalizable vertex operators:

$$
\begin{array}{ll}
R_{-++}^{0}=\varphi e^{-\phi / 2+i\left(-H+H^{1}+H^{2}\right) / 2+\varphi / Q}, & R_{+-+}^{0}=\varphi e^{-\phi / 2+i\left(H+H^{1}-H^{2}\right) / 2+\varphi / Q} \\
R_{++-}^{0}=\varphi e^{-\phi / 2-i\left(-H+H^{1}-H^{2}\right) / 2+\varphi / Q}, & R_{---}^{0}=\varphi e^{-\phi / 2-i\left(H+H^{1}+H^{2}\right) / 2+\varphi / Q} .
\end{array}
$$

These four operators ${ }^{17}$ are mapped by the supercharges into the tachyon (6.3), schematically $Q_{\alpha} R_{+.}^{0} .=T^{+}$and $Q_{\dot{\alpha}} R_{-. .}^{0}=T^{-}$.

\subsubsection{Closed superstring}

The closed superstring spectrum is obtained as the product of holomorphic and antiholomorphic vertex operators subject to level matching and mutual locality conditions. We want the Type IIB spectrum here, so the GSO projection in the antiholomorphic sector is the same as in the holomorphic one.

The allowed combinations are

$$
T^{ \pm} \bar{T}^{ \pm}, R^{0} \bar{T}^{ \pm}, T^{ \pm} \bar{R}^{0}, R^{0} \bar{R}^{0}, G_{\mu \nu} \equiv J_{\mu} \bar{J}_{\nu}, J_{\mu} \bar{R}^{1}, R^{1} \bar{J}_{\nu}, R^{1} \bar{R}^{1} .
$$

These degrees of freedom can be arranged in supermultiplets as follows. The supergravity multiplet can be depicted as

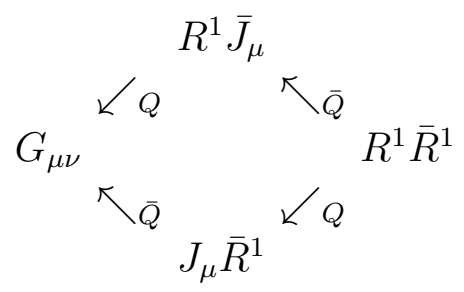

It contains $32 \oplus 32$ states and is off-shell because for the zero-momentum case the transversality condition does not impose any restriction on the sign of $H^{I}$.

Then we have the supermultiplet in which the tachyon sits, which is an off-shell $\mathcal{N}=2 S O(1,3)$ gauge multiplet. Since we are working at zero momentum in the $S O(1,3)$ directions, we have to mod out the vertex operators by the center of the group, namely we identify $R_{++-}^{0} \sim R_{+-+}^{0}$ and $R_{+++}^{0} \sim R_{+--}^{0}$. Then we can depict the multiplet as

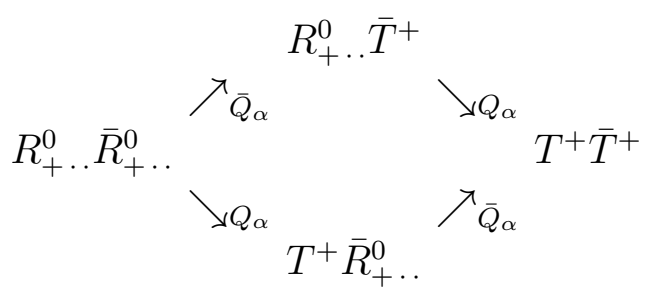

The full supermultiplet consists of other three parts, which are generated by acting with $Q_{\dot{\alpha}}$ and $\bar{Q}_{\dot{\alpha}}$ on $R_{-}^{0} . . \bar{R}_{-}^{0}$. and $R_{ \pm}^{0} . . \bar{R}_{\mp}^{0} \ldots$. In total we have $8 \oplus 8$ states, which represent an off-shell $\mathcal{N}=2 S O(1,3)$ supercurrent.

\footnotetext{
${ }^{17}$ In terms of a full $S O(6)$ spinor representation, these $\mathrm{R}$ states have an even number of +'s and correspond to a $\underline{4} \in S U(4) \simeq S O(6)$.
} 


\subsection{Pure spinor variables}

The RNS bosonic space-time coordinates are $x, \varphi, x^{\mu}$. We proceed as above to map the RNS to a patch of the pure spinor space. The first step is to realize, following [4] that there exist another set of four supercharges, which we denote by $q_{\dot{+}_{\alpha}}$ and $q_{\dot{+} \dot{\alpha}}$, which are mutually local with respect to the ones in (6.1), are conserved, but not BRST invariant. Their explicit form is given in the Appendix.

The six dimensional pure spinor consists of two complex Weyl spinors $\lambda_{i}^{A}$, for $i=1,2$, in the $\underline{4}$ of $S U(4)=S O(6)$. The pure spinor constraint is written using the $4 \times 4$ off-diagonal antisymmetric Pauli matrices of the six dimensional Dirac matrices as $\epsilon^{i j} \lambda_{i}^{A} \sigma_{A B}^{m} \lambda_{j}^{B}=0$. In terms of $U(3)$ representations, that we will conveniently use in the map, each pure spinor splits into a singlet and a vector $\lambda_{i}^{A}=\left(\lambda_{i}^{+}, \lambda_{i}^{a}\right)$, for $a=1,2,3$, and we can rewrite the pure spinor constraint as

$$
\epsilon^{i j} \lambda_{i}^{+} \lambda_{j}^{a}=0, \quad \epsilon_{a b c} \epsilon^{i j} \lambda_{i}^{a} \lambda_{j}^{b}=0 .
$$

We refer to the appendix for the relations between all the different spinor representations. Working in the patch $\lambda_{1}^{+} \neq 0$ we can solve the pure spinor constraint as $\lambda_{2}^{a}=\frac{\lambda_{2}^{+}}{\lambda_{1}^{+}} \lambda_{1}^{a}$ and see that a pure spinor in six dimensions has five independent components [29]. We map the RNS variables to this patch of the pure spinor space. However, since the map is quite similar to the case $d=2$ in section 5.2, we present the details in the Appendix. Here we only quote the results.

The RNS stress tensor, with the appropriate addition of a $c=0$ quartet, is mapped to the following pure spinor stress tensor

$$
\begin{array}{r}
T^{\prime}=-\frac{1}{2} \partial x^{\mu} \partial x^{\nu} \eta_{\mu \nu}-\frac{1}{2}(\partial \varphi)^{2}-\frac{1}{2}\left(\partial x^{\prime}\right)^{2}+\frac{Q}{2} \partial^{2}\left(\varphi-i x^{\prime}\right) \\
-p_{I \alpha} \partial \theta^{I \alpha}-p_{I \dot{\alpha}} \partial \theta^{I \dot{\alpha}}+w_{I \alpha} \partial \lambda^{I \alpha}+w_{I \dot{\alpha}} \partial \lambda^{I \dot{\alpha}}-\frac{1}{2} \partial^{2} \log \Omega
\end{array},
$$

where $I=+, \dot{+}$ and $I=+$ stands for the physical supercoordinates, while $I=\dot{+}$ denotes the unphysical ones. The indices $(\alpha, \dot{\alpha})$ refer to the Weyl and anti-Weyl spinor indices of $S O(1,3)$ Lorentz symmetry. $\Omega$ is the holomorphic top form on the pure spinor space, on the patch $\lambda^{+i} \neq 0$ it reads

$$
\Omega=\left(\lambda^{+1}\right)^{-3} .
$$

The six dimensional pure spinor constraint, written according to the space-time $S O(1,3)$ Lorentz symmetry, reads

$$
\begin{aligned}
\delta_{I J} \lambda^{I \alpha} \lambda^{J \dot{\alpha}} & =0, \\
\epsilon_{I J} \epsilon_{\alpha \beta} \lambda^{I \alpha} \lambda^{J \beta} & =0, \\
\epsilon_{I J} \epsilon_{\dot{\alpha} \dot{\beta}} \lambda^{I \dot{\alpha}} \lambda^{J \dot{\beta}} & =0,
\end{aligned}
$$

Mapping the RNS saturation rule for the amplitudes on the sphere to the pure spinor variables one gets a requirement for an insertion of $\left(\lambda^{+}\right)^{3}$, which is consistent with the measure corresponding to the top form we obtained. 


\subsubsection{Supersymmetry algebra}

We consider just the holomorphic sector in the following. In the RNS formalism we have eight conserved supercurrents, out of which only four are physical. We want to find their algebra. As above we take $q_{+i}$ in the $+\frac{1}{2}$ picture and all the rest in the

$-\frac{1}{2}$ picture and compute their OPE's. If we take these OPE's and map them to the pure spinor variables, we find the following algebra

$$
\begin{aligned}
q_{+\alpha}(z) q_{+\dot{\alpha}}(0) & \sim \frac{1}{z} \sigma_{\alpha \dot{\alpha}}^{m} \partial x_{m}(0), \quad q_{\dot{+} \alpha}(z) q_{\dot{+} \dot{\alpha}}(0) \\
& \sim \frac{1}{z} \sigma_{\alpha \dot{\alpha}}^{m} \partial x_{m}(0), \\
q_{+\alpha}(z) q_{\dot{+\beta}}(0) \sim \frac{\epsilon_{\alpha \beta} Q}{z^{2}}+\frac{\epsilon_{\alpha \beta}}{z} \partial(\varphi-i x)(0), q_{+\dot{\alpha}}(z) q_{\dot{+} \dot{\beta}}(0) & \sim \frac{\epsilon_{\dot{\dot{\beta}} \dot{\beta}} Q}{z^{2}}+\frac{\epsilon_{\dot{\alpha} \dot{\beta}}}{z} \partial(\varphi-i x)(0),
\end{aligned}
$$

and $q_{+\alpha} q_{\dot{+} \dot{\alpha}} \sim 0, q_{+\dot{\alpha}} q_{\dot{+} \alpha} \sim 0$. We see that the same story follows as in the $d=0$ and $d=2$ cases. There are two sets of supercharges, the physical $(+)$ and the unphysical $(\dot{+})$ ones, which separately close on a flat four dimensional supersymmetry algebra. However, the cross OPE's between the physical and the unphysical sets have an anomalous double pole. This means that the non anomalous current algebra realizes the $S O(1,3)$ space-time supersymmetry.

We want to construct GS-like constraints that reproduce the algebra (6.11) with opposite overall signs, as usual. They are the direct generalization of the four dimensional ones in (5.27)

$$
\begin{aligned}
& d_{I \alpha}=p_{I \alpha}-\frac{1}{2} \delta_{I J}\left[\partial x^{m}-\frac{1}{4} f^{m}(\theta)\right]\left(\sigma_{m} \theta^{J}\right)_{\alpha}-\frac{1}{2} \epsilon_{\alpha \beta}\left[\tau_{I J} \theta^{J \beta} \partial(\varphi-i x)-\epsilon_{I J} Q \partial \theta^{J \beta}\right], \\
& d_{I \dot{\alpha}}=p_{I \dot{\alpha}}-\frac{1}{2} \delta_{I J}\left[\partial x^{m}-\frac{1}{4} f^{m}(\theta)\right]\left(\theta^{J} \sigma_{m}\right)_{\dot{\alpha}}-\frac{1}{2} \epsilon_{\dot{\alpha} \dot{\beta}}\left[\tau_{I J} \theta^{J \dot{\beta}} \partial(\varphi-i x)-\epsilon_{I J} Q \partial \theta^{J \dot{\beta}}\right],
\end{aligned}
$$

where we introduced the notation $f^{m}(\theta)=\delta_{I J}\left(\partial \theta^{I} \sigma^{m} \theta^{J}-\theta^{I} \sigma^{m} \partial \theta^{J}\right)$, to save space. We are using the two by two Pauli matrices $\gamma_{\alpha \dot{\alpha}}^{m}$ of the $S O(1,3)$ Lorentz group. The $d$ 's realize the $q$ 's algebra (6.11) we obtained from the RNS map, but with opposite signs

$$
d_{I \alpha}(z) d_{J \dot{\alpha}}(0) \sim-\epsilon_{I J} \frac{\epsilon_{\alpha \beta} Q}{z^{2}}-\delta_{I J} \frac{\sigma_{\alpha \dot{\alpha}}^{m} \Pi_{m}(0)}{z}-\tau_{I J} \frac{\epsilon_{\alpha \beta}}{z} \partial(\varphi-i x)(0),
$$

and all the others vanish.

Let us discuss the space-time symmetries. To identify the quantum numbers of the superderivates, we refer to their RNS origin.

$$
\begin{array}{ccc} 
& \multicolumn{3}{c}{S O(1,3) \times U(1)_{x}} \\
d_{+\alpha} & \left(\frac{1}{2}, 0\right) & +1 \\
d_{\dot{+} \dot{\alpha}} & \left(0, \frac{1}{2}\right) & +1 \\
d_{\dot{+} \alpha} & \left(\frac{1}{2}, 0\right) & -1 \\
d_{+\dot{\alpha}} & \left(0, \frac{1}{2}\right) & -1
\end{array}
$$


In the RNS description, even if all the $d$ 's are conserved currents, only half of them are actually physical. In the pure spinor, due to the double pole in the OPE's (6.13), the worldsheet current algebra only realizes $\mathcal{N}=1$ supersymmetry in four dimensions in the holomorphic sector. The physical supersymmetry is the one generated by

$$
d_{+\alpha}(z) d_{+\dot{\alpha}}(0) \sim-\frac{\sigma_{\alpha \dot{\alpha}}^{m} \Pi_{m}(0)}{z}
$$

The closed superstring will realize $\mathcal{N}=2$ four dimensional supersymmetry.

\subsubsection{Cohomology}

The pure spinor BRST charge in the six dimensional non-critical superstring is

$$
Q_{B}=\oint\left(\lambda^{I \alpha} d_{I \alpha}+\lambda^{I \dot{\alpha}} d_{I \dot{\alpha}}\right)
$$

where the GS-like constraint in the linear dilaton background are (6.12). The strategy to compute the cohomology will be the same as in the previous cases. First we compute the cohomology in the enlarged superspace, containing all the $\theta^{I \alpha}$ and $\theta^{I \dot{\alpha}}$, then we will restrict to the physical ones $\theta^{+}$, that enter in the supersymmetry current algebra, and drop the $\theta^{\dot{+}}$.

Due to the OPE's (6.13), the nilpotency of the BRST charge requires the following conditions on the pure spinors

$$
\begin{gathered}
\delta_{I J} \lambda^{I \alpha} \lambda^{J \dot{\alpha}}=0, \\
\epsilon_{\alpha \beta} \lambda^{+\alpha} \lambda^{\dot{+} \beta}+\epsilon_{\dot{\alpha} \dot{\beta}} \lambda^{+\dot{\alpha}} \lambda^{\dot{+} \dot{\beta}}=0, \\
\epsilon_{\alpha \beta}\left(\partial \lambda^{+\alpha} \lambda^{\dot{+} \beta}-\lambda^{+\alpha} \partial \lambda^{\dot{+} \beta}\right)+\epsilon_{\dot{\alpha} \dot{\beta}}\left(\partial \lambda^{+\dot{\alpha}} \lambda^{\dot{+} \dot{\beta}}-\lambda^{+\dot{\alpha}} \partial \lambda^{\dot{+} \dot{\beta}}\right)=0,
\end{gathered}
$$

The first two conditions are directly implied by the pure spinor constraint (6.10). The last derivative condition is implied by the pure spinor constraint as well. To show this, we derive the Ward Identities as in [13, 26] and we prove that the derivative constraints (6.19) are implied by the other constraints.

It will be convenient to use an $S U(4)$ notation for the six dimensional spinors, see the appendix for the details. We start by noting that the product of two pure spinors $\lambda_{i}^{A}$, that belong to $(\underline{4}, \underline{2})$ representation of $S U(4) \times S O(2)$, can be decomposed into representations $(\underline{10}, \underline{3}) \oplus(\underline{10}, \underline{1}) \oplus(\underline{6}, \underline{3}) \oplus(\underline{6}, \underline{1})$. Moreover, since the pure spinors are commuting variables only the representations $(\underline{10}, \underline{3}) \oplus(\underline{6}, \underline{1})$ survive. The vector representation of $S O(6)$ is present only in $(\underline{6}, \underline{1})$ and it gives the correct pure spinor constraint. The latter can be written as follows

$$
\lambda_{i}^{[A} \lambda_{j}^{B]}=0, \quad i, j=1,2
$$


since for $(\underline{6}, \underline{3})$ is automatically satisfied. ${ }^{18}$ It implies that $\lambda_{i}^{[A} \partial \lambda_{j}^{B]} \epsilon^{i j}=0$, and we will use the Ward Identities to prove also $\lambda_{(i}^{[A} \partial \lambda_{j)}^{B]}=0$.

The pure spinor constraints (6.10) imply the gauge invariance of the conjugated variables $\delta w_{A i}=\Lambda_{A B} \epsilon_{i j} \lambda^{B j}$ (where $\Lambda_{[A B]} \in(\underline{6}, \underline{0})$ is the gauge parameter) and it can be shown that the only gauge invariant combinations are $J_{(i j)}=\frac{1}{2}\left(w_{A i} \lambda_{j}^{A}+w_{A j} \lambda_{i}^{A}\right)$ and $J_{A}{ }^{B}=w_{A i} \lambda^{B i}$ (notice that they are in the representations $(\underline{0}, \underline{3}),(\underline{15}, \underline{0}) \oplus(\underline{1}, \underline{0})$, where the trace of the second operator is the ghost charge). Following [13, 26], using the free OPE's for the pure spinor and their conjugates, one finds

$$
\begin{gathered}
: J_{(i k)} \lambda_{j}^{A} \lambda^{k B}:-\frac{1}{2}: J_{C}^{B} \lambda_{j}^{A} \lambda_{i}^{C}:= \\
=\frac{\alpha^{\prime}}{2}\left(\lambda_{j}^{A} \partial \lambda_{i}^{B}-\epsilon_{i j} \lambda_{k}^{A} \partial \lambda^{k B}-\lambda_{i}^{A} \partial \lambda_{j}^{B}+\lambda_{j}^{B} \partial \lambda_{i}^{A}\right) .
\end{gathered}
$$

Antisymmetrizing over the indices $A$ and $B$ and using (6.20) (which implies also that $: J_{C}^{[B} \lambda_{j}^{A]} \ldots:=0$ under the normal ordering sign) one can conclude that $\lambda_{i}^{[A} \partial \lambda_{j}^{B]}=0$ for any $i, j$. In particular we get $\lambda_{(i}^{[A} \partial \lambda_{j)}^{B]}=0$ which is a stronger version of the derivative constraints (6.19).

Let us now look at the cohomology. Following the strategy of the previous $d=0$ and $d=2$ cases, there will be two kinds of vertex operators contributing to the lowest lying cohomology at ghost number one. The first kind is the usual weight zero operator

$$
\mathcal{U}^{(1)}=\lambda^{I \alpha} A_{I \alpha}\left(\bar{Z}, x^{\mu}, \theta\right)+\lambda^{I \dot{\alpha}} A_{I \dot{\alpha}}\left(\bar{Z}, x^{\mu}, \theta\right) .
$$

We are not presenting the details of the computation, which are not very illuminating, ${ }^{19}$ but just state the results. Imposing that

$$
Q_{B} \mathcal{U}^{(1)}=0, \quad \delta \mathcal{U}^{(1)}=Q_{B} \Omega^{(0)},
$$

gives $8 \oplus 8$ states, whose superfields depend both on the physical $\theta^{+}$and the unphysical $\theta^{\dot{+}}$. By considering only the operators that belong to the physical supersymmetry current algebra (6.15), namely the ones containing only $\theta^{+}$, we get $4 \oplus 4$ states. The closed string spectrum is given by the product of the holomorphic and antiholomorphic vertex operators and at the end of the day we get $32 \oplus 32$ states which arrange in the $\mathcal{N}=2$ off-shell four-dimensional supergravity multiplet, which reproduces the RNS computation.

The second kind of vertex operator is the one in which the massless tachyon sits. This is the six-dimensional generalization of the massive vertex operator that

\footnotetext{
${ }^{18}$ In the four dimensional case ( $d=2$ in our notations), we have the pure spinor constraints $\lambda \Gamma^{m} \lambda=0$ where $m=1, \ldots, 4$, but, due to the commuting nature of $\lambda$ 's, also the constraint $\lambda \Gamma^{m} \Gamma^{5} \lambda=0$ is trivially satisfied. From the first constraint, it follows that $\lambda \Gamma^{m} \partial \lambda=0$. In addition from the Ward Identities one can prove that $\lambda \gamma^{m} \gamma^{5} \partial \lambda=0$ [26].

${ }^{19}$ The cohomology computation for the vertex operators $(6.22)$ is totally analogous to the flat six dimensional case considered in 26, 28].
} 
we used for two-dimensional and four-dimensional superstrings. We leave the details of this computations for a future analysis.

\subsubsection{Curved non-critical backgrounds}

We follow the discussion in section 4.3 .3 and suggest a generalization of the noncritical pure spinors to generic six dimensional curved backgrounds with at most sixteen real supercharges. The computations are very similar, so we just present the covariantized linear dilaton action for the matter part

$$
\begin{gathered}
S=\frac{1}{2 \pi \alpha^{\prime}} \int d^{2} z\left(\frac{1}{2} G_{M N}(Y) \partial Y^{M} \bar{\partial} Y^{N}+E_{M}^{i A}(Y) d_{i A} \bar{\partial} Y^{M}+E_{M}^{i \hat{A}}(Y) \bar{d}_{i \hat{A}} \partial Y^{M}\right) \\
-\int d^{2} z r^{(2)} \Phi(Y)
\end{gathered}
$$

where we introduced the curved six dimensional supercoordinates $Y^{M}=\left(x^{m} ; \theta^{i A}, \bar{\theta}^{i \hat{A}}\right)$. Note that $m$ is a curved six dimensional vector index, while $A$ is a curved six dimensional Weyl index and $i=1,2$ enumerates different Weyl spinors. The E's are the vielbein superfields. In the linear dilaton case, the background superfields take the following values: the supervielbeins are the six dimensional flat ones, the dilaton

superfield is linear $\Phi=\frac{Q}{2}(\varphi-i x)$, the metric $G_{M N}$ is constant, but in addition to the usual flat components we have a new flat spinorial part, whose nonvanishing components in four dimensional Weyl notations are

$$
\begin{array}{ll}
G_{I \alpha, J \beta}=-Q \epsilon_{I J} \epsilon_{\alpha \beta}, & G_{I \dot{\alpha}, J \dot{\beta}}=-Q \epsilon_{I J} \epsilon_{\dot{\alpha} \dot{\beta}}, \\
G_{I \hat{\alpha}, J \hat{\beta}}=-Q \epsilon_{I J} \epsilon_{\hat{\alpha} \hat{\beta}}, & G_{I \hat{\dot{\alpha}}, J \hat{\beta}}=-Q \epsilon_{I J} \epsilon_{\dot{\alpha} \hat{\dot{\beta}}},
\end{array}
$$

which is proportional to the background charge $Q=\sqrt{2}$. We regard this as a specific feature of the linear dilaton superspace structure in the pure spinor formalism. This explicitly breaks the original $S O(6)$ Lorentz invariance of the action to $S O(4)$, while preserving the $U(1)_{x} \mathrm{R}$ symmetry.

It is suggestive to think of (6.24) as the matter part of the non-critical pure spinor action in a generic curved six dimensional background.

\subsubsection{Anomalies}

The pure spinor degrees of freedom are two Weyl spinors and anti Weyl spinors $\left(\lambda^{I \alpha}, \lambda^{I \dot{\alpha}}\right)$, for $I=+, \dot{+}$ with the condition

$$
\begin{aligned}
\delta_{I J} \lambda^{I \alpha} \lambda^{J \dot{\alpha}} & =0, \\
\epsilon_{I J} \epsilon_{\alpha \beta} \lambda^{I \alpha} \lambda^{J \beta} & =0, \\
\epsilon_{I J} \epsilon_{\dot{\alpha} \dot{\beta}} \lambda^{I \dot{\alpha}} \lambda^{J \dot{\beta}} & =0,
\end{aligned}
$$

These six equations define a five dimensional space with a conical singularity at $\lambda^{I \alpha}=\lambda^{I \dot{\alpha}}=0 \forall I, \alpha, \dot{\alpha}$. Again, we expect that the correct way to cure the singularity 
is by just removing it. We leave for the future the proof that this procedure yields a pure spinor space with vanishing first Chern class and first Pontryagin class. This pure spinor space is different from the two and four-dimensional cases we discussed above, in that the manifold we obtain after the removal of the singularity at the origin is still connected. This resembles the critical ten-dimensional pure spinor space structure.

\section{The pure spinor measure}

In this section we will comment on the computation of tree level string theory amplitudes in the non-critical pure spinor formalism. In this formalism, a crucial issue in the evaluation of string amplitudes is to construct a proper prescription for the integration of the zero modes of the pure spinor variables. In ten dimensions, this prescription was introduced by Berkovits in [5] and further discussed in [31. However, in the case of lower dimensional pure spinor theories [29, 28], it is not yet clear how to define this prescription for the zero mode integration measure. In the following, we will present such a prescription in the case of the non-critical type II superstrings.

Before proceeding, let us make some comments on the interpretation of our integration measure. In the linear dilaton background, which is the main subject of this paper, the string perturbation theory is not well defined, due to the strong coupling regime. Therefore, the linear dilaton prescription that we will discuss only makes sense once we properly define the calculation in the strong coupling regime, for instance by introducing the Liouville interaction, or by replacing the $\left(x^{\prime}, \varphi\right)$ part of the space with a cigar CFT. On the other hand, just as the flat ten-dimensional prescription is the starting point for generalization to curved backgrounds, we expect this lower dimensional prescription to be useful to study non-critical superstrings on other curved backgrounds in which string perturbation theory is well defined.

In the RNS formalism, the zero mode prescription for the ghosts in a tree level amplitude is dictated by

$$
\left\langle c \partial c \partial^{2} c e^{-2 \phi} e^{Q \varphi}\right\rangle=1 .
$$

We would like to obtain the analogous prescription in the pure spinor variables for non-critical superstrings. The simplest way to do that is to use the maps from the RNS variables to the pure spinor variables in various dimensions. Doing that we obtain a generic saturation rule $\left\langle\lambda^{3} \theta^{d / 2} e^{Q(\varphi-i x)}\right\rangle$, where $d$ is the number of flat directions in which the supersymmetry is realized, that we can list explicitely

$$
\left\langle\lambda^{3} \theta e^{2(\varphi-i x)}\right\rangle_{d=0}, \quad\left\langle\lambda^{3} \theta^{2} e^{\sqrt{3}(\varphi-i x)}\right\rangle_{d=2}, \quad\left\langle\lambda^{3} \theta^{3} e^{\sqrt{2}(\varphi-i x)}\right\rangle_{d=4},
$$

where $x$ denotes $x^{\prime}$ for simplicity of notation. The $e^{Q(\varphi-i x)}$ term is required to soak up the background charge $Q$. Also we see that we need three ghost number one vertex operators for a nonvanishing tree level amplitude. 
Consider next a definition analgous to the pure spinor measure for the critical superstrings [31]. Let us recall first the critical case. The ghost number anomaly reads

$$
J_{g h}(z) T(0) \sim \frac{Q_{g h}}{z^{3}}+\ldots,
$$

with $Q=-8$. The generic pure spinor measure is $d^{11} \lambda$ as the pure spinor space is eleven-dimensional. One writes this measure as

$$
d^{11} \lambda=[\mathcal{D} \lambda] \lambda^{3},
$$

where $[\mathcal{D} \lambda]$ is a Lorentz invariant measure with ghost charge $-Q_{g h}=8$, and we are left with three additional factors of $\lambda$.

Consider now the measure for the superspace variables $\theta^{\alpha}$. In ten dimensions we have 16 supercharges, therefore the integration measure is $d^{16} \theta$. We need to insert as many picture changing operators $Y=C_{\alpha} \theta^{\alpha} \delta\left(C_{\alpha} \lambda^{\alpha}\right)$ as the number of independent components of the pure spinor $\lambda^{\alpha}$, where $C_{\alpha}$ are irrelevant constant spinors [31]. There are eleven picture changing insertions, one for each of the eleven components of the pure spinor, and since every PCO $Y$ carries a factor of $\theta$, we are left with

$$
d^{16} \theta Y^{11} \sim d^{16} \theta \theta^{11} \delta^{11}(\lambda) \sim d^{5} \theta \delta^{11}(\lambda) .
$$

In this way we get that the tree level pure spinor measure is defined by $\left\langle\lambda^{3} \theta^{5}\right\rangle=1$ :

$$
d^{11} \lambda d^{16} \theta Y^{11} \sim[\mathcal{D} \lambda]_{-Q_{g h}} \lambda^{3} d^{5} \theta \quad \Rightarrow \quad\left\langle\lambda^{3} \theta^{5}\right\rangle=1 .
$$

Consider next the non-critical superstrings. The number of pure spinor degrees of freedom and the ghost number anomaly in the various non-critical dimensions $d+2$ is

$$
\begin{array}{cccc} 
& d=0 & d=2 & d=4 \\
\operatorname{dim}_{\mathbb{C}}(\mathcal{M}) & 1 & 2 & 5 \\
Q_{g h} & 2 & 1 & -2
\end{array}
$$

Following the critical superstrings case, we propose that we can write the pure spinor integration measure as

$$
\begin{array}{cccc} 
& d=0 & d=2 & d=4 \\
\text { measure } & d \lambda=[\mathcal{D} \lambda]_{-2} \lambda^{3} & d^{2} \lambda=[\mathcal{D} \lambda]_{-1} \lambda^{3} & d \lambda=[\mathcal{D} \lambda]_{2} \lambda^{3}
\end{array}
$$

Consider now the integration over the superspace coordinates $\theta$. As we noted before, in the pure spinor superstring (as well as in the hybrid formalism [四), the number of fermionic coordinates is doubled with respect to the RNS formalism. Thus, we get the following integration over the superspace

$$
\begin{array}{cccc} 
& d=0 & d=2 & d=4 \\
\text { superspace } & d^{2} \theta & d^{4} \theta & d^{8} \theta
\end{array}
$$


Now we have to insert in the amplitude as many picture changing operators $Y=$ $C \theta \delta(C \lambda)$ as the number of independent components of the pure spinor, and since each PCO carries a factor of $\theta$ we get

$$
\begin{aligned}
d=0: & d^{2} \theta Y & =d^{2} \theta \theta \delta(\lambda)=d \theta \delta(\lambda), \\
d=2: & d^{4} \theta Y^{2} & =d^{4} \theta \theta^{2} \delta^{2}(\lambda)=d^{2} \theta \delta^{2}(\lambda), \\
d=4: & d^{8} \theta Y^{5} & =d^{8} \theta \theta^{5} \delta^{5}(\lambda)=d^{3} \theta \delta^{5}(\lambda),
\end{aligned}
$$

We see that in this way we reobtain the same prescription for the pure spinors and $\theta$ 's in tree level amplitudes as the one obtained by the direct map (7.1) from the RNS measure.

\section{Curved backgrounds}

As we noted in the introduction, due to the presence of a cosmological constant type term which vanishes only for $d=10$, the low energy approximation $E \ll l_{s}^{-1}$ is not valid for non-critical superstrings. Indeed, the higher order curvature terms $\left(l_{s}^{2} \mathcal{R}\right)^{n}$ cannot be discarded.

One may write an action for the lightest fields (which are always massive), whose bosonic part takes the form

$$
S=\frac{1}{2 k_{d}^{2}} \int d^{d} x \sqrt{G}\left(e^{-2 \Phi}\left(R+4(\partial \Phi)^{2}+\frac{10-d}{\alpha^{\prime}}-\frac{1}{2 \cdot 3 !} H^{2}\right)-\frac{1}{2 \cdot n !} F_{n}^{2}\right),
$$

where we have not included the "non-tachyonic" tachyon field. Of course, solutions to the field equations will have string scale curvature.

An interesting class of backgrounds of type IIA non-critical superstrings are $A d S_{d}$ spaces with a constant dilaton $e^{2 \Phi}=\frac{1}{N_{c}^{2}}$ and a $d$-form RR field $F_{d}$

$$
l_{s}^{2} F_{d}^{2}=2(10-d) d ! N_{c}^{2} .
$$

The background has a string scale scalar curvature $l_{s}^{2} \mathcal{R}=d-10$. While this backgrounds cannot be studied via supergravity, it can be studied in our pure spinor formalism. Let us sketch some of the details.

As we have seen in the previous sections, a basic feature of the pure spinor formalism is a doubling of the superspace. This we did by enlarging the linear dilaton superspace structure to include BRST non-invariant superspace coordinates and their conjugate momenta. In the linear dilaton background, working in a doubled superspace in the pure spinor variables required an appropriate projection to the physical superspace. However, the doubled superspace allows us to study pure spinor superstrings in backgrounds with twice as many supersymmetries as in the linear dilaton background. 
For instance, the non-critical superstring on $A d S_{4}$ with $N_{c}$ units of RR four form flux $F_{4}$ is obtained from the supercoset

$$
\frac{O S p(2 \mid 4)}{S O(1,3) \times S O(2)}
$$

and has eight real supercharges, which is precisely the content of the doubled superspace in the non-critical superstring on the four-dimensional linear dilaton background $\mathbb{R}^{1,1} \times \mathbb{R}_{\varphi} \times U(1)_{x}$. The supercharges are Majorana spinors $Q_{I}^{\alpha}, I=1,2$, $\alpha=1, \ldots, 4$. We can decompose the Majorana index $\alpha$ into two Weyl indices $a, \dot{a}$ and we identify the supercharges $Q_{1}^{a}, Q_{1}^{\dot{a}}$ with the left moving sector charges and $Q_{2}^{a}, Q_{2}^{\dot{a}}$ with those of the right-moving sector. The symmetry $S O(2)$ acts as the R-symmetry on the space.

The $O S p(2 \mid 4)$ left invariant 1-form is expanded in the basis of generators of the supergroup (following Metsaev and Tseytlin [32]) as $L_{\mu} P^{\mu}+L_{\mu \nu} J^{\mu \nu}+L_{I J} \Lambda^{I J}+L_{\alpha}^{I} Q_{I}^{\alpha}$. The pure spinors action then consists of three terms

$$
S_{p s}=S_{G S}+S_{\kappa}+S_{g h}
$$

where the first term is the $\kappa$-symmetric GS action [3]

$$
S_{G S}=\int_{\Sigma} d^{2} z \eta_{\mu \nu} L^{\mu} \bar{L}^{\nu}+\int_{M_{3}} d^{3} y \epsilon^{I J} L^{\mu} L_{J}^{\alpha}\left(\gamma_{5} \gamma_{\mu}\right)_{\alpha \beta} L_{J}^{\beta},
$$

where $\Sigma=\partial M_{3}$ and we work in the conformal gauge on the worldsheet. The second term is

$$
S_{\kappa}=\int_{\Sigma} d^{2} z\left(\delta^{i j}+i \epsilon^{i j}\right) d_{\alpha i} \bar{L}_{j}^{\alpha}+\left(\delta^{i j}-i \epsilon^{i j}\right) \bar{d}_{\alpha i} L_{j}^{\alpha}+q_{R R} \bar{d}_{a i} \gamma^{5 \alpha \beta} d_{\beta j} \delta^{i j} .
$$

It contains the kinetic term for the fermions (recall that the first line does not give a good kinetic term for the $\theta$ 's because of the $\kappa$-symmetry) and the coupling with the RR field. The RR 4-form of the $A d S_{4}$ background produces a bispinor of the form $\delta_{i j} \gamma_{\alpha \beta}^{\mu \nu \rho \sigma} F_{\mu \nu \rho \sigma}$ which can be written as $\delta_{i j} \gamma_{\alpha \beta}^{5} q_{R R}$, where $q_{R R}=\epsilon^{\mu \nu \rho \sigma} F_{\mu \nu \rho \sigma}$ is the constant flux.

The third term in the action

$$
\begin{aligned}
S_{g h}=\int_{\Sigma} & d^{2} z\left(\delta^{i j}+i \epsilon^{i j}\right) w_{\alpha i} \bar{\partial} \lambda_{j}^{\alpha}+\left(\delta^{i j}-i \epsilon^{i j}\right) \bar{w}_{\alpha i} \partial \bar{\lambda}_{j}^{\alpha} \\
& +N_{\mu \nu} \bar{L}^{\mu \nu}+N_{i j} \bar{L}^{i j}+\bar{N}_{\mu \nu} L^{\mu \nu}+\bar{N}_{i j} L^{i j} \\
& +N_{\mu \nu} \bar{N}_{\rho \sigma}\left(-4 \eta^{\mu[\rho} \eta^{\sigma] \nu}\right)+N_{i j} \bar{N}_{k l} \eta^{i[k} \eta^{l] j}
\end{aligned}
$$

contains the free action for the pure spinor ghost fields and the interaction with the Lorentz generators of $S O(1,3)$ and of $S O(2)$ and in the last line the coupling with the Riemann tensor is also described. The value of the Riemann tensor is easily given by the fact that the background is coset manifold. 
The BRST operator reads

$$
Q=\int d \sigma\left(\lambda_{I}^{\alpha} d_{\alpha}^{I}\right)
$$

where the eight pure spinor variables $\lambda_{I}^{\alpha}$ satisfy the pure spinor conditions (5.34) we used in the description of the four-dimensional linear dilaton background, both in the left and in the right moving sectors. The $d$ 's are the ones computed from the action (8.4).

One needs to show that the BRST operator is conserved and nilpotent on the four-dimensional pure spinor constraints and that this sigma model on $A d S_{4}$ is a consistent string theory background at all orders in the worldsheet perturbation theory, along the lines of [6]. A complete analysis of non-critical superstrings on $A d S_{4}$ will appear in a future publication.

\section{Discussion and open problems}

In the paper we presented a pure spinor formalism to describe non-critical superstrings. We explicitly constructed the pure spinor description of the non-critical superstrings in a linear dilaton background, which can be further used to study more general backgrounds. We have shown that, by mapping the bosonic and fermionic linear dilaton RNS variables to pure spinor variables, we get a description of a patch of the pure spinor space. A basic requirement of the map is a doubling of the superspace. We achieved this by enlarging the linear dilaton superspace structure to include superspace coordinates and their conjugate momenta, which are not BRST invariant on the RNS side, although they are conserved. Working in a doubled superspace in the pure spinor variables required an appropriate projection to the physical superspace.

We consider the doubled superspace as a feature, since it allows us to study pure spinor superstrings in backgrounds with twice as many supersymmetries as the linear dilaton ones. As a concrete example we presented the action for the type IIA non-critical superstring on $A d S_{4}$ with RR four form flux, described by the supercoset

$$
O S p(2 \mid 4) /(S O(1,3) \times S O(2)),
$$

that has eight real supercharges, which is double the supersymmetry of the noncritical superstring on the linear dilaton background $\mathbb{R}^{1,1} \times \mathbb{R}_{\varphi} \times U(1)_{x}$. We leave for a future work the proof of the consistency of this background, along the lines of [6], and the study of its spectrum and its holographic interpretation. We just point out that this formulation of the non-critical superstring raises the possibility of addressing a new class of non-critical holographic backgrounds, such as the ones proposed by Polyakov [3], which up to now have not been accessible to worldsheet tools. 
It would also be interesting to consider also the non-critical pure spinor superstring on the eight-dimensional linear dilaton background

$$
\mathbb{R}^{1,5} \times \mathbb{R}_{\varphi} \times U(1)_{x}
$$

The construction of the worldsheet pure spinor formulation can be repeated along the lines of the lower dimensional cases and we expect it to be straightforward.

The realization of the pure spinor variables $\left(\lambda^{\alpha}, w_{\alpha}\right)$ as a beta-gamma system living on a curved pure spinor space has important consequences. This has been discussed in [7, 8]. By using a field redefinition from RNS to pure spinor variables, we confirmed this ten-dimensional analysis, by computing the modifications of the stress tensor due to the holomorphic top form on the pure spinor space, as well as saturation rules for tree level correlators. We then extended this map to the noncritical pure spinor spaces and analyzed the global obstructions to define the pure spinor system on the worldsheet and on space-time, reflected by quantum anomalies in the worldsheet and pure spinor space holomorphic diffeomorphisms. The noncritical pure spinor spaces have a singularity at $\lambda^{\alpha}=0$. Removing the origin left a non-anomalous theory. However, for non-critical superstrings in two and four dimensions, this resulted in a disconnected pure spinor space.

There are various other open issues that deserve further study. We have not performed a complete analysis of the BRST cohomology in the pure spinor formalism. In the ten-dimensional case, the argument for the equivalence between the RNS and pure spinor cohomologies used in an essential way a similarity transformation [15]. It would be of importance to find such similarity transformations in the various noncritical dimensions as well. A direct map of the RNS unintegrated vertex operators $V$ reveals the interesting structure

$$
V \sim \lambda P_{\theta}(\theta, \partial \theta, \ldots) P_{p}(p, \partial p, \ldots)
$$

with the $P$ 's being some polynomials and RNS GSO projection being implemented automatically. However, for most vertex operators the direct map does not give the pure spinor vertex operators in the simple form expected. Here we expect to see again the importance of the similarity transformation.

In the two-dimensional superstrings, there is a special set of operators in the BRST cohomology at spin zero and ghost number zero, known as the ground ring [33, 23]. In the pure spinor formulation of two-dimensional type II non-critical string, as discussed in section 4 , the cohomology at ghost number zero and weight zero is not empty: it contains several operators constructed as explained in (4.49). It would be interesting to explore this further.

Another issue is the removal of the pure spinor constraints [34. It has been shown in in [35] that removing the pure spinor constraint might lead to an infinite tower of ghost-for-ghosts, and it seems that, except a finite number of them, the 
rest of the ghost-for-ghosts are the same in all dimensions (see [36]). It would be interesting to see if the removal of the pure spinor constraint in lower dimensions leads to the same ghost-for-ghosts and what are the differences.

A crucial issue that requires further study is the projection from the doubled superspace to the physical linear dilaton superspace. We expect this to be of much importance also for the study of pure spinor critical superstrings on backgrounds with less than maximal supersymmetry. Indeed, a similar lack of understanding exists for instance when trying to study pure spinor superstrings compactified on Calabi-Yau manifolds. ${ }^{20}$ A possible way to gain insight into this problem would be to consider the ten-dimensional pure spinor superstring on $\mathbb{R}^{1,5} \times \mathbb{R}^{4} / \mathbb{Z}_{2}$ and understand how the twisted states arise in the cohomology computation.

Finally, we note that we discussed in this paper only the non-normalizable vertex operators. It is clearly of importance to analyze the normalizable vertex operators in the pure spinor formalism ${ }^{21}$.

\section{Acknowledgments}

We would like to thank O. Aharony, N. Berkovits, A. Giveon, N. Itzhaki, M. Kroyter, D. Kutasov, S. Murthy, N. Nekrasov, J. Sonnenschein and E. Witten for discussions. LM would like to thank in particular S. Murthy for enjoyable explanations. This work is supported in part by DIP (grant H.52) and ISF (grant number 03200306). LM is supported by the EU Contract MRTN-CT-2004-512194.

\section{A. Notations}

In this appendix we summarize for the convenience of the reader the notations that we use in the construction of the non-critical pure spinor superstrings on $\mathbb{R}^{1, d-1} \times$ $\mathbb{R}_{\varphi} \times U(1)_{x}$.

There are three relevant groups : The Lorentz $S O(1, d-1)$ group of symmetries of $\mathbb{R}^{1, d-1}$, the $S O(d+2)$ group of symmetries of $\mathbb{R}^{1, d+1}$ and $U\left(\frac{d+2}{2}\right)$.

The target space coordinates are denoted by

$$
\begin{array}{ccc}
x^{m}, & m=0, \ldots, d-1, x, \varphi, & (d+2)-\text { dimensional vector } \\
x^{\mu}, & \mu=0, \ldots, d-1, & \text { flat } d-\text { dimensional vector }
\end{array}
$$

We denote by $\Gamma^{m}, m=1, \ldots, d+2$, the $(d+2)$-dimensional Dirac matrices.

\footnotetext{
${ }^{20}$ In appendix $\mathrm{F}$ we present an example of a possible way to perform a projection. However, this is not the linear dilaton background case.

${ }^{21}$ For a recent analysis of normalizable states in the linear dilaton background see [37].
} 
In order to describe the doubling of superspace we introduce an index $I=+, \dot{+}$ that keeps track of the physical and unphysical fermionic coordinates

$$
I=\left\{\begin{array}{l}
+\quad \text { physical superspace } \\
\dot{+} \text { unphysical superspace. }
\end{array}\right\}
$$

Consider next the different cases.

$\mathbb{R}_{\varphi} \times U(1)_{x}$

The two-dimensional superstring has no spacetime Lorentz symmetry, that is $d=0$. The only index is $I$.

$\mathbb{R}^{1,1} \times \mathbb{R}_{\varphi} \times U(1)_{x}$

The four-dimensional superstring has an $S O(1,1)$ Lorentz symmetry, that is $d=2$. The fermionic coordinates we that we use in the pure spinor formulation are four Majorana-Weyl spinors $\theta^{I i}$. The two physical fermionic coordinates, $I=+$, $i=1,2$, have the same chirality. The two unphysical supercoordinates, $I=\dot{+}$, $i=1,2$, have the same chirality, but opposite to that of the physical ones. Therefore, in this case the index $I=+, \dot{+}$ takes care of the spinor chirality as well.

In the text we passed from this $S O(1,1)$ notation to the $S O(4)$ notation. In the $S O(4)$ notation we reshuffle the fermionic coordinates into a Dirac spinor $\theta^{A}$, $A=1, \ldots, 4$, that splits into a pair of a Weyl and an anti-Weyl spinors: $\theta^{\alpha}$ in the $(\underline{2}, 0) \in S O(4)$ and $\theta^{\dot{\alpha}}$ in the $(0, \underline{2}) \in S O(4) .{ }^{22}$ The mapping of the indices goes as follows

$$
\begin{array}{ll}
(I, i)=(+, 1) \rightarrow \alpha=1, & (I, i)=(+, 2) \rightarrow \dot{\alpha}=\dot{1}, \\
(I, i)=(\dot{+}, 1) \rightarrow \alpha=2, & (I, i)=(\dot{+}, 2) \rightarrow \dot{\alpha}=\dot{2} .
\end{array}
$$

Finally, in the map from the RNS we used the $U(2)$ notations, where an $S O(4)$ Dirac spinor $\left(\lambda^{\alpha}, \lambda^{\dot{\alpha}}\right)$ decomposes into $\left(\lambda^{+}, \lambda^{a}, \lambda_{a b}\right)$ of $U(2)$, where $a=1,2$ and $\lambda_{a b}$ is the antisymmetric representation with only one component. The relation between this and the Weyl notation is

$$
\lambda^{\alpha}=\lambda^{a}, \quad \lambda^{\dot{\alpha}}=\left(\lambda^{+}, \lambda_{a b}\right) .
$$

The four-dimensional pure spinor constraint can be cast in the following different ways

$$
\begin{array}{cc}
S O(4): & \lambda^{A} \Gamma_{A B}^{m} \lambda^{B} \Rightarrow \lambda^{\alpha} \lambda^{\dot{\alpha}}=0, \\
U(2): & \lambda^{+} \lambda^{a}=0, \quad \lambda_{a b} \lambda^{a}=0, \\
S O(1,1): & \tau_{i j} \lambda^{I i} \lambda^{J j}=0 . \\
\mathbb{R}^{1,3} \times \mathbb{R}_{\varphi} \times U(1)_{x} &
\end{array}
$$

\footnotetext{
${ }^{22}$ Since a Weyl spinor is complex, to recover the correct degrees of freedom this has to be understood in the closed string picture.
} 
The six-dimensional superstring has an $S O(1,3)$ Lorentz symmetry, that is $d=4$. The fermionic coordinates that we use in the pure spinor formulation are two copies of four dimensional Dirac spinors $\left(\lambda^{I \alpha}, \lambda^{I \dot{\alpha}}\right)$, for $I=+, \dot{+}$. The two physical fermionic coordinates, $I=+$, are a Weyl $\lambda^{+\alpha}$ and an anti-Weyl $\lambda^{+\dot{\alpha}}$ spinor. The same applies for the two unphysical supercoordinates, $\lambda^{\dot{+} \alpha}$ and $\lambda^{\dot{+} \dot{\alpha}}$.

In the text we pass from this $S O(1,3)$ notation to the space $S O(6)=S U(4)$ notation. In the $S U(4)$ notation we reshuffle the fermionic coordinates into two Weyl spinors $\lambda_{i}^{A}$ in the $\underline{4} \in S U(4)$, for $A=1, \ldots, 4$ and $i=1,2$. Here the index $i$ simply enumerates different $S U(4)$ Weyl spinors with the same chirality. The mapping of the indices goes as follows

$$
\lambda_{1}^{A}=\left(\lambda^{+\alpha}, \lambda^{\dot{+} \dot{\alpha}}\right), \quad \lambda_{2}^{A}=\left(\lambda^{\dot{+} \alpha}, \lambda^{+\dot{\alpha}}\right) .
$$

The reason for this is the match of their R-charge, as shown in (6.14).

Finally, in the map from the RNS we use the $U(3)$ notations. First, in the pure spinor formulation we use two Weyl spinors $\lambda_{i}^{A}$ of $S U(4)$ that decompose into $U(3)$ representations according to $\lambda_{i}^{A}=\left(\lambda_{i}^{+}, \lambda_{i}^{a}\right)$, for $i=1,2$, where $\lambda^{+}$is a $U(3)$ singlet and $\lambda^{a}$ is a $U(3)$ vector. We can fit the $S O(1,3)$ spinors into these $U(3)$ representations as follows

$$
\begin{aligned}
& \left(\lambda_{1}^{+}, \lambda_{1}^{a}\right) \quad \in U(3): \quad \lambda_{1}^{+} \quad \lambda_{1}^{1} \lambda_{1}^{2} \lambda_{1}^{3} . \\
& \left(\lambda^{+\dot{\alpha}}, \lambda^{\dot{+} \alpha}\right) \in S O(1,3): \quad \lambda^{+\dot{1}} \lambda^{\dot{+1}} \lambda^{\dot{+} 2} \lambda^{+\dot{2}} \\
& \left(\lambda_{2}^{+}, \lambda_{2}^{a}\right) \quad \in U(3): \quad \lambda_{2}^{+} \quad \lambda_{2}^{1} \quad \lambda_{2}^{2} \quad \lambda_{2}^{3} \\
& \left(\lambda^{+\alpha}, \lambda^{\dot{+} \dot{\alpha}}\right) \in S O(1,3): \quad \lambda^{\dot{+}}-\lambda^{+1}-\lambda^{+2} \lambda^{\dot{+} \dot{2}}
\end{aligned}
$$

There is a little abuse of notation here: the + denotes the singlet representations of $U(3)$, while on the $S O(1,3)$ it denotes the $I=+$ physical superspace.

The six-dimensional pure spinor constraint can be cast in the following ways

$$
\begin{array}{cc}
U(3): & \epsilon^{i j} \lambda_{i}^{+} \lambda_{j}^{a}=0, \quad \epsilon_{a b c} \epsilon^{i j} \lambda_{i}^{a} \lambda_{j}^{b}=0, \\
S O(1,3): & \delta_{I J} \lambda^{I \alpha} \lambda^{J \dot{\alpha}}=0, \\
& \epsilon_{I J} \epsilon_{\alpha \beta} \lambda^{I \alpha} \lambda^{J \beta}=0, \\
& \epsilon_{I J} \epsilon_{\dot{\alpha} \dot{\beta}} \lambda^{I \dot{\alpha}} \lambda^{J \dot{\beta}}=0 .
\end{array}
$$

\section{B. Non-critical RNS superstrings}

In this appendix we collect the RNS notations in $d=2 n$ flat dimensions (corresponding to a $2 n+2$ dimensional background).

\section{B.1 The matter system}

The matter stress energy tensor of the system reads

$$
\begin{gathered}
T_{m}=\sum_{\mu=1}^{2 n}\left(-\frac{1}{2}\left(\partial x^{\mu}\right)^{2}-\frac{1}{2} \psi^{\mu} \partial \psi^{\mu}\right)-\frac{1}{2}(\partial x)^{2}-\frac{1}{2} \psi^{x} \partial \psi^{x}+ \\
-\frac{1}{2}(\partial \varphi)^{2}+\frac{Q(n)}{2} \partial^{2} \varphi-\frac{1}{2} \psi_{l} \partial \psi_{l}
\end{gathered}
$$


The OPE's conventions that we will be using are

$$
\begin{gathered}
x^{i}(z) x^{j}(0) \sim-\eta^{i j} \log z, \quad \varphi(z) \varphi(0) \sim-\log z, \\
\psi^{i}(z) \psi^{j}(0) \sim \frac{\eta^{i j}}{z}, \quad \psi_{l}(z) \psi_{l}(0) \sim \frac{1}{z} . \\
T(z) e^{r x}(0) \sim\left(\frac{-r^{2} / 2}{z^{2}}+\frac{\partial}{z}\right) e^{r x}(0), \quad T(z) e^{s \varphi}(0) \sim\left(\frac{-s(s-Q(n)) / 2}{z^{2}}+\frac{\partial}{z}\right) e^{s \varphi}(0) .
\end{gathered}
$$

We define $\Psi=\psi_{l}+i \psi$ and $\Psi^{I}=\psi^{I}+i \psi^{I+n}$ (with $I=1, \ldots, n$ ). These are bosonized in the usual way by introducing the bosonic fields $H, H^{I}$ :

$$
\begin{aligned}
\Psi & =\sqrt{2} e^{i H}, \quad \Psi^{I}=\sqrt{2} e^{i H^{I}}, \\
\Psi \Psi^{\dagger} & =2 i \partial H, \quad \Psi \Psi^{I \dagger}=2 i \partial H^{I},
\end{aligned}
$$

where $\uparrow$ denotes Hermitian conjugation in field space without interchanging left- and right-movers: $\Psi^{\dagger}=\psi-i \psi_{l}, \Psi^{\dagger I}=\psi^{I}-i \psi^{I+n}$. We have

$$
H^{I}(z) H^{J}(0) \sim-\delta^{I J} \log z, \quad H(z) H(0) \sim-\log z .
$$

We define the spin fields $\Sigma^{ \pm}=e^{ \pm \frac{i}{2} H}$ and $\Sigma^{a}=e^{ \pm \frac{i}{2} H^{1} \ldots \pm \frac{i}{2} H^{n}}$, where the index $a$ runs over the independent spinor representation of $S O(2 n)$.

\section{B.2 The ghost system}

We have a fermionic $(b, c)$ ghost system of weights $(2,-1)$ and a bosonic $(\beta, \gamma)$ ghost system of weights $\left(\frac{3}{2},-\frac{1}{2}\right)$. The OPE's and stress-energy tensor are

$$
\begin{aligned}
c(z) b(0) & \sim \frac{1}{z}, \quad \gamma(z) \beta(0) \sim \frac{1}{z}, \\
T_{\text {ghost }} & =-2 b \partial c-\partial b c-\frac{3}{2} \beta \partial \gamma-\frac{1}{2} \partial \beta \gamma,
\end{aligned}
$$

Let us bosonize the ghost systems. We define

$$
c=e^{\chi}, \quad b=e^{-\chi},
$$

with

$$
\begin{gathered}
T_{b, c}=T_{\chi}=\frac{1}{2}(\partial \chi)^{2}+\frac{3}{2} \partial^{2} \chi \\
\chi(z) \chi(0) \sim \log z \\
T_{\chi}(z) e^{a \chi}(0) \sim\left(\frac{a(a-3) / 2}{z^{2}}+\frac{\partial}{z}\right) e^{a \chi}(0) .
\end{gathered}
$$


For the superghosts, we have

$$
\begin{gathered}
\gamma=e^{\phi} \eta, \beta=\partial \xi e^{-\phi} \\
T_{\beta, \gamma}=T_{\phi}+T_{\eta, \xi}=-\frac{1}{2}(\partial \phi)^{2}-\partial^{2} \phi-\eta \partial \xi \\
\phi(z) \phi(0) \sim-\log z, \quad \eta(z) \xi(0) \sim \frac{1}{z}, \\
T_{\phi}(z) e^{b \phi}(0) \sim\left(\frac{-b(b+2) / 2}{z^{2}}+\frac{\partial}{z}\right) e^{b \phi}(0) .
\end{gathered}
$$

We further bosonize the fermions into

$$
\eta=e^{\kappa}, \quad \xi=e^{-\kappa},
$$

and

$$
\begin{gathered}
T_{\eta, \xi}=T_{\kappa}=\frac{1}{2}(\partial \kappa)^{2}-\frac{1}{2} \partial^{2} \kappa \\
\kappa(z) \kappa(0) \sim \log z \\
T_{\kappa}(z) e^{c \kappa}(0) \sim\left(\frac{c(c+1) / 2}{z^{2}}+\frac{\partial}{z}\right) e^{c \kappa}(0) .
\end{gathered}
$$

\section{B.3 Supersymmetry}

\section{B.3.1 The $N=2$ superconformal algebra}

In addition to $(\mathbb{B} .1)$ the $N=2$ superconformal algebra includes the supercurrents

$$
\begin{aligned}
& G^{+}=\frac{i}{\sqrt{2}} \sum_{I=1}^{n} \Psi^{I \dagger} \partial\left(x^{I}+i x^{I+n}\right)+\frac{i}{\sqrt{2}} \Psi^{\dagger} \partial(\varphi+i x)-\frac{i Q}{\sqrt{2}} \partial \Psi^{\dagger}, \\
& G^{-}=\frac{i}{\sqrt{2}} \sum_{I=1}^{n} \Psi^{I} \partial\left(x^{I}-i x^{I+n}\right)+\frac{i}{\sqrt{2}} \Psi \partial(\varphi-i x)-\frac{i Q}{\sqrt{2}} \partial \Psi
\end{aligned}
$$

and the $U(1)$ current

$$
J=\frac{1}{2} \sum_{I=1}^{n} \Psi^{I \dagger} \Psi^{I}+\frac{1}{2} \Psi^{\dagger} \Psi+i Q \partial x .
$$

In terms of the fermion bosonization these currents take the form

$$
\begin{aligned}
G^{+} & =i \sum_{I=1}^{n} e^{-i H^{I}} \partial\left(x^{I}+i x^{I+n}\right)+i e^{-i H} \partial(\varphi+i x+i Q H), \\
G^{-} & =i \sum_{I=1}^{n} e^{i H^{I}} \partial\left(x^{I}-i x^{I+n}\right)+i e^{i H} \partial(\varphi-i x-i Q H), \\
J & =-i \sum_{I=1}^{n} \partial H^{I}-i \partial H+i Q \partial x
\end{aligned}
$$




\section{B.3.2 The twisted $N=2$ algebra}

Out of the matter and ghost superconformal generators we can construct a twisted $\hat{c}=2 N=2$ superconformal algebra whose generators are

$$
\begin{array}{r}
G^{++}=\gamma G_{m}+c\left(T_{m}-\frac{3}{2} \beta \partial \gamma-\frac{1}{2} \gamma \partial \beta-b \partial c\right)-\gamma^{2} b+\partial^{2} c+\partial(c \xi \eta), \\
G^{-}=b, \quad J^{\prime}=c b+\eta \xi, \quad T^{\prime}=T_{\text {matter }}+T_{\text {ghost }},
\end{array}
$$

where $G_{m}=G_{+}+G_{-}$is the sum of the two matter supercurrents. The dimension one current $G^{\prime+}$ is the BRST current of the RNS superstring and $J^{\prime}$ is the ghost current.

\section{The RNS spectra}

In this appendix we show the details of the computation of the spectrum in the RNS formalism on linear dilaton backgrounds. We are interested just in operators that do not break space-time supersymmetry. These are the primary operators of the worldsheet $N=2$ superconformal algebra.

\section{C. $1 \mathrm{~d}=2$}

Let us discuss the spectrum on $\mathbb{R}^{1,1} \times \mathbb{R}_{\varphi} \times U(1)_{x}$. First we consider the holomorphic sector and then we match holomorphic and antiholomorphic sectors to get the closed superstring, for the type IIB case.

\section{C.1.1 Holomorphic sector}

NS sector

The tachyon vertex operator in the -1 picture is

$$
T=e^{-\phi+i k_{\mu} x^{\mu}+i p x+\beta \varphi},
$$

and the GSO projection requires $Q p \in 2 \mathbb{Z}+1$. The condition $\Delta(T)=1$ reads

$$
k_{\mu}^{2}-\left(\beta-\frac{Q}{2}\right)^{2}+p^{2}+\frac{Q^{2}}{4}-1=0,
$$

and the lowest lying state has $p= \pm \frac{1}{Q}$. The operator

$$
T_{ \pm}=e^{-\phi+\frac{1}{Q}(\varphi \pm i x)},
$$

is a worldsheet (anti)chiral primary $\Delta_{\text {matter }}\left(T_{ \pm}\right)= \pm \frac{q}{2}=\frac{1}{2}$ annihilated by $G^{ \pm}$, with space-time R-charge $R= \pm \frac{2}{3}$. It turns out that $T_{+}$and $T_{-}$are not mutually local. However, we are interested in the mutual locality only when matching holomorphic and antiholomorphic sectors, so we will discuss locality only below. 
The other NS operators are analogous to the "vectors" in the ten dimensional superstring

$$
\begin{aligned}
& J^{ \pm}=e^{-\phi \pm H+i k_{\mu} x^{\mu}+i p x+\beta \varphi}, \\
& J^{\mu}=e^{-\phi \pm H_{1}+i k_{\mu} x^{\mu}+i p x+\beta \varphi},
\end{aligned}
$$

where $\mu$ is an $S O(1,1)$ Lorentz vector index. The GSO projection requires $Q p \in 2 \mathbb{Z}$, and $\Delta=1$ gives $k_{\mu}^{2}+p^{2}-(\beta-Q / 2)^{2}+Q^{2} / 4=0$. The lowest lying states with $p=\beta=0$ are $J^{\mu}=e^{-\phi \pm H_{1}}$, in particular they are worldsheet $N=2$ primaries (they have only single poles with $G^{ \pm}$) and are not charged under $U(1)_{x}$.

\section{$R$ sector}

The operators in the Ramond sector are

$$
R=e^{-\frac{\phi}{2}+\frac{i}{2} \epsilon H+\frac{i}{2} \epsilon_{1} H_{1}+i k_{\mu} x^{\mu}+i p x+\beta \varphi},
$$

where $\epsilon, \epsilon_{1}= \pm 1$. The GSO projection requires $Q p \in 2 \mathbb{Z}+\frac{1}{2}$ for $\epsilon=\epsilon_{1}= \pm 1$ and $Q p \in 2 \mathbb{Z}-\frac{1}{2}$ for $\epsilon=-\epsilon_{1}= \pm 1$. The $\Delta(R)=1$ condition fixes $k_{\mu}^{2}+p^{2}-(\beta-Q / 2)^{2}=0$. Then we need to impose the Dirac equation (4.14), which fixes separately for each direction the sign of the momentum according to the sign of the corresponding spin component. In the $\left(x^{1}, x^{2}\right)$-plane we find $i k_{1}=\epsilon_{1} k_{2}$, while in the $(x, \varphi)$-plane we have $\beta=Q / 2+\epsilon p$. The last condition, together with the Seiberg bound $\beta \leq Q / 2$, imposes a restriction on the allowed momenta in the $x$ direction. Introducing the notation $R_{\epsilon, \epsilon_{1}}$ we find

$$
\begin{aligned}
& R_{++}=e^{-\frac{1}{2} \phi+\frac{i}{2}\left(H+H_{1}\right)+i k_{\mu} x^{\mu}-i p x+\left(\frac{Q}{2}-p\right) \varphi}, p=\frac{1}{Q}\left(2 n+\frac{3}{2}\right) \geq 0, \\
& R_{--}=e^{-\frac{1}{2} \phi-\frac{i}{2}\left(H+H_{1}\right)+i k_{\mu} x^{\mu}+i p x+\left(\frac{Q}{2}-p\right) \varphi}, p=\frac{1}{Q}\left(2 n+\frac{1}{2}\right) \geq 0, \\
& R_{+-}=e^{-\frac{1}{2} \phi+\frac{i}{2}\left(H-H_{1}\right)+i k_{\mu} x^{\mu}-i p x+\left(\frac{Q}{2}-p\right) \varphi}, p=\frac{1}{Q}\left(2 n+\frac{1}{2}\right) \geq 0, \\
& R_{-+}=e^{-\frac{1}{2} \phi-\frac{i}{2}\left(H-H_{1}\right)+i k_{\mu} x^{\mu}+i p x+\left(\frac{Q}{2}-p\right) \varphi}, p=\frac{1}{Q}\left(2 n+\frac{3}{2}\right) \geq 0,
\end{aligned}
$$

and we note that the $R_{--}$operator is non local with respect to (C.1). We are interested in the lowest lying components of the $\mathrm{R}$ vertex operators at zero momentum $k_{\mu}=0$ in the transverse $\mathbb{R}^{1,1}$ directions, which are listed in the main text in (5.5).

\section{C.1.2 Closed superstring}

We match left and right vertex operators in IIB for concreteness, the antiholomorphic sector being a copy of the holomorphic one we just described. In type IIB we have two dimensional $\mathcal{N}=(4,0)$ spacelike SUSY in the flat noncompact directions. Because of the requirement of mutual locality of the vertex operators, the spectrum is not just the left right product of the sectors. Let us denote each vertex operators in the left sector by $(\alpha, F)$, where $\alpha$ is the space-time fermion index, 0 in the $N S$ and 1 in 
the $\mathrm{R}$ and $F$ is the worldsheet spinor index, given by the sum of the picture plus the eigenvalues of the Lorentz Cartan generators. ${ }^{23}$ In addition we have the momentum $n$ and winding $w$ in the compact $x$ direction. A closed string vertex operator is denoted by $(\alpha, F, \bar{\alpha}, \bar{F})$ and $(n, w)$. Following [30, 18], the mutual locality condition reads

$$
F_{2} \alpha_{1}-F_{1} \alpha_{2}-\bar{F}_{1} \bar{\alpha}_{2}+\bar{F}_{2} \bar{\alpha}_{1}+\frac{1}{2}\left(\alpha_{1} \alpha_{2}-\bar{\alpha}_{1} \bar{\alpha}_{2}\right)+2\left(n_{1} w_{2}+n_{2} w_{1}\right) \in 2 \mathbb{Z} .
$$

The total $U(1) \mathrm{R}$ charge of a closed string vertex operator is the sum of the left and right charges. We are again interested in the short space-time $\mathcal{N}=(4,0)$ supermultiplets.

$N S-N S$ sector

The tachyon, denoted by $(\alpha, F, \bar{\alpha}, \bar{F})=(0,-1,0,-1)$, has momenta $p_{L, R}=$ $\frac{1}{Q}\left(n \pm \frac{3 w}{2}\right) \in \frac{1}{Q}(2 \mathbb{Z}+1)$ and the $\Delta=1$ condition gives

$$
k_{\mu}^{2}-\left(\beta-\frac{Q}{2}\right)^{2}+\left(\frac{n}{Q}\right)^{2}+\left(\frac{Q w}{2}\right)^{2}+\frac{Q^{2}}{4}-1=0 .
$$

The lowest lying tachyons are (5.6).

The other NS-NS operators with $(0,0,0,0)$ are the left right product of the states in (C.4)

$$
\begin{aligned}
G_{ \pm \pm} & =e^{-\phi-\bar{\phi} \pm i H \pm i \bar{H}+i k_{\mu} x^{\mu}+i p_{L} x+i p_{R} \bar{x}+\beta \varphi} \\
G_{\mu \pm} & =e^{-\phi-\bar{\phi} \pm i H_{1} \pm i \bar{H}+i k_{\mu} x^{\mu}+i p_{L} x+i p_{R} \bar{x}+\beta \varphi} \\
G_{ \pm \mu} & =e^{-\phi-\bar{\phi} \pm i H \pm i \bar{H}_{1}+i k_{\mu} x^{\mu}+i p_{L} x+i p_{R} \bar{x}+\beta \varphi} \\
G_{\mu \nu} & =e^{-\phi-\bar{\phi} \pm i H_{1} \pm i \bar{H}_{1}+i k_{\mu} x^{\mu}+i p_{L} x+i p_{R} \bar{x}+\beta \varphi}
\end{aligned}
$$

where $\mu, \nu=1,2$ are $S O(1,1)_{L}$ Lorentz vector indices, the momenta are $p_{L, R} \in \frac{1}{Q} 2 \mathbb{Z}$ and we introduced the notation $J \bar{J} \equiv G$. The mass shell condition is $k_{\mu}^{2}+\left(\frac{n}{Q}\right)^{2}+$ $\left(\frac{Q w}{2}\right)^{2}-(\beta-Q / 2)^{2}+Q^{2} / 4=0$. The mutual locality condition between the NSNS states is given by $n_{1} w_{2}+n_{2} w_{1} \in \mathbb{Z}$. We are interested in the lowest lying states among (C.4) that belong to a short space-time multiplet. At zero momentum in the transverse directions we find (5.7).

The windings and momenta can be non integer. Indeed, they may not have an interpretation in terms of actual windings and momenta in the $x$ direction, but are just a useful notation for simplifying the mutual locality computation.

\section{$R-R, R-N S$ and $N S-R$ sectors}

The matching of the lowest lying operators in these sectors has been shown in the main text.

\footnotetext{
${ }^{23}$ E.g. the vertex $e^{-\frac{1}{2} \phi+\frac{i}{2} H-\frac{i}{2} H_{1}}$ has $F=-1 / 2+1 / 2-1 / 2=-1 / 2 . F$ is defined only modulo 2 .
} 
C.2 $d=4$

Here we discuss some details of the $S O(1,3)$ spectrum of the six dimensional superstring. First let us collect the second set of physical supercharges, which are nonlocal with respect to the ones in (6.1)

$$
\begin{array}{ll}
q_{-1}=e^{-\frac{1}{2} \phi+i\left(H^{1}-H^{2}+H-Q x\right) / 2}, & q_{-2}=e^{-\frac{1}{2} \phi+i\left(-H^{1}+H^{2}+H-Q x\right) / 2} \\
q_{-i}=e^{-\frac{1}{2} \phi+i\left(H^{1}+H^{2}-H+Q x\right) / 2}, & q_{-\dot{2}}=e^{-\frac{1}{2} \phi+i\left(-H^{1}-H^{2}-H+Q x\right) / 2}
\end{array} .
$$

The choice of $q_{+}$in the holomorphic sector and $q_{-}$in the antiholomorphic defines the type IIA superstring. However, we will stick to the type IIB case.

\section{C.2.1 Holomorphic sector}

The first NS state is the tachyon whose vertex operator is

$$
T_{p}=e^{-\phi+i k_{\mu} x^{\mu}+i p x+\beta \varphi} .
$$

From requiring it to be of weight $\Delta(T)=1$ we obtain the condition

$$
k_{\mu} k^{\mu}+p^{2}-\beta(\beta-Q)=1
$$

and mutual locality with the chosen set of supercharges requires $p Q \in 2 \mathbb{Z}+1$. It is a primary field and for it to be a chiral primary of the $N=2 \mathrm{SCA}$ it must satisfy

$$
k_{1}=-i k_{3}, \quad k_{2}=-i k_{4}, \quad p=\beta
$$

while the same relations with opposite signs will give an anti-chiral primary.

Restricting to $k_{\mu}=0$ states with the lowest R-charge we have $\beta=\frac{1}{Q}$ and $p= \pm \frac{1}{Q}$. $\beta$ saturates the Seiberg bound $\beta \leq \frac{Q}{2}$ [24], the vertex operator (C.11) is normalizable and does not represent a microscopic state and we have to replace it with the non-normalizable state ${ }^{24}$

$$
T_{p}=\varphi e^{-\phi+i k_{\mu} x^{\mu}+i p x+\beta \varphi}
$$

Note that the lowest lying mode of this vertex operator has $\beta=p$, so that the condition $\Delta(T)=1$ gives $k_{\mu}^{2}=0$. This is different from the $d=2$ case: there, with respect to the flat $S O(1,1)$ momenta, the tachyon was off-shell with a continuous mass above a gap. Here, the tachyon is again off-shell, but now the lowest value for the mass is zero. The tachyon being massless is a specific feature of the $d=4$ case. The two lowest lying such tachyons are given in (6.3).

\footnotetext{
${ }^{24}$ We can understand the appearance of the factor $\varphi$ in (C.14) by the fact that this is the nonnormalizable solution of the Laplace equation in six dimensions.
} 
At the next level there are the NS vectors with one NS oscillator excitation given by

$$
J_{\mu}=e^{-\phi+i \epsilon H^{I}+i k_{\mu} x^{\mu}+i p x+\beta \varphi}
$$

with the $\Delta(J)=1$ condition

$$
k_{\mu} k^{\mu}+p^{2}-\beta(\beta-Q)=0 .
$$

Mutual locality with the supercharges requires that $p Q \in 2 \mathbb{Z}$. In order for the vertex operator to represent a physical state the momentum must satisfy $k_{I}=-i \epsilon k_{I+2}$ which is nothing more than the transversality condition. The zero momentum states are given in (6.4). The vector polarized in the $\varphi x$-plane is

$$
J_{ \pm}=e^{-\phi+i \epsilon H+i k_{\mu} x^{\mu}+i p x+\beta \varphi}
$$

with the same conditions for having weight one and being mutually local with the supercharges and $\beta=\epsilon p+Q$. From these requirements and the bound $\beta \leq \frac{Q}{2}$ it follows that such states always have momentum in $\mathbb{R}^{1,3}$ so they do not appear in the zero momentum cohomology.

We now turn to the Ramond sector. The $\mathrm{R}$ ground state is of the form

$$
R=e^{-\phi / 2+i\left(\epsilon_{1} H^{1}+\epsilon_{2} H^{2}+\epsilon H\right) / 2+i p x+\beta \varphi},
$$

where $k_{\mu} k^{\mu}+p^{2}=\left(\beta-\frac{Q}{2}\right)^{2}$. The $F=1$ states are mutually local with the supercharges for $p Q \in 2 \mathbb{Z}+1$ while for the $F=0$ states the $x$ momentum is $p Q \in 2 \mathbb{Z}$. From the Dirac equation one obtains

$$
k_{1}=-i \epsilon_{1} k_{3}, \quad k_{2}=-i \epsilon_{2} k_{4}, \quad \beta=\epsilon p+\frac{Q}{2} .
$$

The zero momentum $\mathrm{R}$ states with $F=1$ are given in (6.5) and the ones with $F=0$ are in $(6.6)$.

\section{C.2.2 Closed superstring}

The closed superstring operators are obtained as the product of holomorphic and antiholomorphic vertex operators subject to level matching and mutual locality conditions. Using the same convention $(\alpha, F, \bar{\alpha}, \bar{F})$ as in the previous sections [18], the mutual locality condition reads now

$$
F_{1} \alpha_{2}-F_{2} \alpha_{1}-\bar{F}_{1} \bar{\alpha}_{2}+\bar{F}_{2} \bar{\alpha}_{1}+\alpha_{1} \alpha_{2}-\bar{\alpha}_{1} \bar{\alpha}_{2}+2\left(n_{1} w_{2}+n_{2} w_{1}\right) \in 2 \mathbb{Z} .
$$

The level matching $L_{0}=\tilde{L}_{0}$ requires

$$
N-\tilde{N}=\frac{\tilde{p}^{2}-p^{2}}{2},
$$

and since we only consider the lowest $\mathrm{R}$-charge states the range of $x$-momenta is $p=0, \pm \frac{1}{Q}$. The zero momentum supermultiplets are quoted in the main text. 


\section{Pure spinor cohomology}

\section{D.1 $d=0$ gauge supermultiplet}

In this appendix we show how the supermultiplet which the tachyon sits in is obtained from the pure spinor cohomology as in (4.50). In order to simplify the notations we define the complex coordinates $Z=\varphi+i x$ and $\bar{Z}=\varphi-i x$ such that $Z(z) \bar{Z}(0) \sim$ $-2 \log z$. We will also use a parametrization of the $d_{I}$ in which

$$
d_{+}=p_{+}-\theta^{\dot{+}} \partial \bar{Z}+Q \partial \theta^{\dot{+}}, \quad d_{\dot{+}}=p_{\dot{+}} .
$$

In order to prevent confusion with the background charge, we will denote the BRST operator by $Q_{B}=\oint \lambda^{I} d_{I}$.

The tachyon appears from what would naively be the first level of massive states, i.e. the weight one ghost number one vertex operator

$$
\mathcal{U}^{(1)}=\partial \lambda^{I} A_{I}+\lambda^{I} \partial \theta^{J} B_{I J}+\lambda^{I} d_{J} C_{I}^{J}+\lambda^{I} \Pi^{\bar{Z}} H_{I}+\lambda^{+} J_{+}^{+} F_{++}^{+}+\lambda^{\dot{+}} J_{\dot{+}}^{\dot{+}} F_{\dot{+}+}^{+},
$$

where $J_{I}^{J}=w_{I} \lambda^{J}$, but where the wavefunctions in the various superfields are all equal to $e^{-Z / Q}$. The above vertex operator then has weight zero. We note that due to the gauge invariance $\delta w_{+}=\Lambda_{+\dot{+}} \lambda^{\dot{+}}+\Lambda_{+\dot{+}}^{\prime} \partial \lambda^{\dot{+}}, \delta w_{\dot{+}}=\Lambda_{\dot{+}+} \lambda^{+}+\Lambda_{\dot{+}+}^{\prime} \partial \lambda^{+}$and the pure spinor constraint the last two terms are the only allowed such terms. The gauge transformation of this operator are given by $\delta \mathcal{U}^{(1)}=Q_{B} \Lambda^{(0)}$, where $\Lambda^{(0)}$ is the ghost number zero operator

$$
\Lambda^{(0)}=\partial \theta^{I} \Omega_{I}+d_{I} \Lambda^{I}+\Pi^{\bar{Z}} \Gamma_{\bar{Z}}+J_{+}^{+} \Phi_{+}^{+}+J_{\dot{+}}^{\dot{+}} \Phi_{\dot{+}}^{\dot{+}} .
$$

The equations of motion obtained by requiring $\mathcal{U}^{(1)}$ to be $Q_{B^{-}}$-closed when the pure spinor constraint and the Ward identities are taken into account are

$$
\begin{aligned}
& D_{+} A_{+}+B_{++}-Q C_{+}^{+}+2 \partial_{Z} C_{+}^{+}=0, \\
& D_{\dot{+}} A_{\dot{+}}+B_{\dot{+}+}+Q C_{\dot{+}}^{+}=0, \\
& D_{+} B_{+I}=D_{\dot{+}} B_{\dot{+} I}=0, \\
& D_{+} H_{+}-C_{+}^{+}=0, \quad D_{\dot{+}} H_{\dot{+}}-C_{\dot{+}}^{+}=0, \\
& D_{+} C_{+}^{+}-F_{++}^{+}=0, \quad D_{\dot{+}} C_{\dot{+}}^{+}-F_{\dot{+}}^{+}=0, \quad D_{+} C_{+}^{\dot{+}}=D_{\dot{+}} C_{\dot{+}}^{+}=0, \\
& D_{+} F_{++}^{+}=D_{\dot{+}} F_{\dot{+}}^{\dot{+}}=0 .
\end{aligned}
$$

These equations of motion are left invariant under the gauge transformations

$$
\begin{aligned}
\delta A_{I} & =\Omega_{I}-\epsilon_{I J} Q \Lambda^{J}+2 \delta_{I}^{+} \partial_{Z} \Lambda^{+}, \\
\delta B_{I J} & =-D_{I} \Omega_{J} \\
\delta C_{I}^{J} & =-D_{I} \Lambda^{J}-\delta_{I}^{+} \delta_{+}^{J} \Phi_{+}^{+}-\delta_{I}^{+} \delta_{\dot{+}}^{J} \Phi_{\dot{+}}^{+}, \\
\delta H_{I} & =-\sigma_{I J}^{1} \Lambda^{J}+D_{I} \Gamma_{\bar{Z}}, \\
\delta F_{++}^{+} & =D_{+} \Phi_{+}^{+}, \quad \delta F_{\dot{+}+}^{+}=D_{\dot{+}} \Phi_{\dot{+}}^{+} .
\end{aligned}
$$


The gauge transformations can be used to choose a gauge in which $A_{I}=0$, $C_{+}^{+}=C_{\dot{+}}^{\dot{+}}=0$ and $H_{I}=0$. In such a gauge the equations of motion yield that

$$
B_{++}=B_{\dot{+}+}=0, \quad C_{+}^{\dot{+}}=C_{\dot{+}}^{+}=0, \quad F_{++}^{+}=F_{\dot{+}+}^{\dot{+}}=0 .
$$

and the only remaining fields are $B_{++}$and $B_{\dot{++}}$ satisfying

$$
D_{+} B_{+\dot{+}}=0=D_{\dot{+}} B_{\dot{+}+},
$$

each with two degrees of freedom. Then we need to project onto the physical supercoordinates $\theta^{+}$, so we keep only $B_{\dot{++}}$ in the cohomology.

The closed string spectrum is a product of the above superfields on the left and

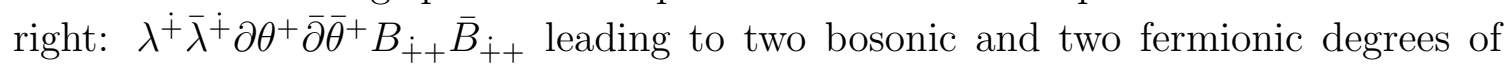
freedom, thus matching the count obtained in the RNS cohomolgy.

\section{D.2 $d=2$ gauge supermultiplet}

In this appendix we show how the four-dimensional tachyon and its supermultiplet are obtained. In order to simplify the computation we use $S O(4)$ spinor notations and the complex coordinates $Z=\varphi+i x, W=x^{1}+i x^{2}$ and their complex conjugates $\bar{Z}$ and $\bar{W}$. The details about how to pass from the $S O(4)$ to the $S O(1,1)$ notations are in appendix A. The superderivatives in these conventions are cast in the form

$$
\begin{aligned}
& d_{\alpha}=p_{\alpha}-\frac{1}{2} \delta_{\alpha \dot{\alpha}} \theta^{\dot{\alpha}} \partial W-\frac{1}{2} \sigma_{\alpha \dot{\alpha}}^{1} \theta^{\dot{\alpha}} \partial \bar{Z}+\frac{Q}{2} \epsilon_{\alpha \dot{\alpha}} \partial \theta^{\dot{\alpha}} \\
& d_{\dot{\alpha}}=p_{\dot{\alpha}}-\frac{1}{2} \delta_{\alpha \dot{\alpha}} \theta^{\alpha} \partial W-\frac{1}{2} \sigma_{\alpha \dot{\alpha}}^{1} \theta^{\alpha} \partial \bar{Z}-\frac{Q}{2} \epsilon_{\alpha \dot{\alpha}} \partial \theta^{\alpha}
\end{aligned}
$$

and their non-singular OPE's read

$$
d_{\alpha}(z) d_{\dot{\alpha}}(0) \sim-\frac{Q \epsilon_{\alpha \dot{\alpha}}}{z^{2}}-\frac{1}{z} \delta_{\alpha \dot{\alpha}} \partial W(0)-\frac{1}{z} \sigma_{\alpha \dot{\alpha}}^{1} \partial \bar{Z}(0) .
$$

The BRST operator is $Q_{B}=\oint\left(\lambda^{\alpha} d_{\alpha}+\lambda^{\dot{\alpha}} d_{\dot{\alpha}}\right)$.

The ghost number one and weight zero vertex operator is obtained with the procedure explained in the previous section. We take the operator one would write for the first massive level in the critical case [26]

$$
\begin{aligned}
\mathcal{U}^{(1)}= & \partial \lambda^{A} A_{A}+\lambda^{A} \partial \theta^{B} B_{A B}+\lambda^{A} d_{B} C_{A}^{B}+\lambda^{A} \Pi^{W} H_{W A}+ \\
& +\lambda^{A} \Pi^{\bar{W}} H_{\bar{W} A}+\lambda^{A} \Pi^{\bar{Z}} K_{\bar{Z} A}+\lambda^{\alpha} J_{\beta}^{\gamma} F_{\alpha \gamma}^{\beta}+\lambda^{\dot{\alpha}} J_{\dot{\beta}}^{\dot{\gamma}} F_{\dot{\alpha} \dot{\gamma}}^{\dot{\beta}},
\end{aligned}
$$

where $A=(\alpha, \dot{\alpha})$ and $B=(\beta, \dot{\beta})$ are $S O(4)$ Dirac indices and $J_{\alpha}^{\beta}=: w_{\alpha} \lambda^{\beta}:$ and $J_{\dot{\alpha}}^{\dot{\beta}}=: w_{\dot{\alpha}} \lambda^{\dot{\beta}}$ : are the worldsheet currents invariant under the gauge transformations in $w_{\alpha}$ and $w_{\dot{\alpha}}$. Then, the wavefunctions of the various superfields appearing in the vertex operator all contain the weight -1 vertex operator $e^{-Z / Q}$, in order to 
have the weight zero vertex operator needed for the massless tachyon. The gauge transformation of this vertex operator is $\delta \mathcal{U}^{(1)}=Q_{B} \Lambda^{(0)}$, where $\Lambda$ is the ghost number zero operator

$$
\Lambda^{(0)}=\partial \theta^{A} \Omega_{A}+d_{A} \Lambda^{A}+\Pi^{W} \Gamma_{W}+\Pi^{\bar{W}} \Gamma_{\bar{W}}+\Pi^{\bar{Z}} \Gamma_{\bar{Z}}+J_{\alpha}^{\beta} \Phi_{\beta}^{\alpha}+J_{\dot{\alpha}}^{\dot{\beta}} \Phi_{\dot{\beta}}^{\dot{\alpha}} .
$$

Using the pure spinor constraint and the Ward identities, $Q_{B} \mathcal{U}^{(1)}=0$ implies the equations of motion

$$
\begin{aligned}
& D_{\beta} A_{\alpha}+B_{\beta \alpha}-\epsilon_{\alpha \dot{\beta}} Q C_{\beta}^{\dot{\beta}}+\delta_{\alpha \dot{\beta}} \partial_{\bar{W}} C_{\beta}^{\dot{\beta}}+\sigma_{\alpha \dot{\beta}}^{1} \partial_{Z} C_{\beta}^{\dot{\beta}}+\delta_{\alpha \dot{\alpha}} \theta^{\dot{\alpha}} H_{W \beta}=0 \\
& D_{\dot{\beta}} A_{\dot{\alpha}}+B_{\dot{\beta} \dot{\alpha}}+\epsilon_{\beta \dot{\alpha}} Q C_{\dot{\beta}}^{\beta}+\delta_{\beta \dot{\alpha}} \partial_{\bar{W}} C_{\dot{\beta}}^{\beta}+\sigma_{\beta \dot{\alpha}}^{1} \partial_{Z} C_{\dot{\beta}}^{\beta}+\delta_{\alpha \dot{\alpha}} \theta^{\alpha} H_{\bar{W} \dot{\beta}}=0 \\
& D_{(\beta} C_{\alpha)}^{\gamma}+F_{(\alpha \beta)}^{\gamma}=0, \quad D_{(\beta} C_{\alpha)}^{\dot{\beta}}=0, \quad D_{(\dot{\beta}} C_{\dot{\alpha})}^{\beta}=0, \quad D_{(\dot{\beta}} C_{\dot{\alpha})}^{\dot{\gamma}}+F_{(\dot{\alpha} \dot{\beta})}^{\dot{\gamma}}=0 \\
& D_{(\alpha} B_{\beta) \gamma}=0, \quad D_{(\alpha} B_{\beta) \dot{\alpha}}-H_{\bar{W}(\alpha} \delta_{\beta) \dot{\alpha}}=0 \\
& D_{(\dot{\alpha}} B_{\dot{\beta}) \dot{\gamma}}=0, \quad D_{(\dot{\alpha}} B_{\dot{\beta}) \alpha}-\delta_{\alpha(\dot{\alpha}} H_{\bar{W} \dot{\beta})}=0 \\
& D_{(\alpha} H_{W \beta)}-C_{(\alpha}^{\dot{\beta}} \delta_{\beta) \dot{\beta}}=0, \quad D_{(\dot{\alpha}} H_{W \dot{\beta})}-\delta_{\beta(\dot{\beta}} C_{\dot{\alpha})}^{\beta}=0 \\
& D_{(\alpha} H_{\bar{W} \beta)}=0, \quad D_{(\dot{\alpha}} H_{\bar{W} \dot{\beta})}=0 \\
& D_{(\alpha} K_{\beta)}-C_{(\alpha}^{\dot{\beta}} \sigma_{\beta) \dot{\beta}}^{1}=0, \quad D_{(\dot{\alpha}} K_{\dot{\beta})}-\sigma_{\beta(\dot{\beta}}^{1} C_{\dot{\alpha})}^{\beta}=0 \\
& D_{(\alpha} F_{\beta \gamma)}^{\delta}=0, \quad D_{(\dot{\alpha}} F_{\dot{\beta} \dot{\gamma})}^{\dot{\delta}}=0
\end{aligned}
$$

which posses the gauge symmetry

$$
\begin{aligned}
\delta A_{\alpha} & =\Omega_{\alpha}-\epsilon_{\alpha \dot{\alpha}} Q \Lambda^{\dot{\alpha}}+\delta_{\alpha \dot{\alpha}} \partial_{\bar{W}} \Lambda^{\dot{\alpha}}+\sigma_{\alpha \dot{\alpha}}^{1} \partial_{Z} \Lambda^{\dot{\alpha}}+\delta_{\alpha \dot{\alpha}} \theta^{\dot{\alpha}} \Gamma_{\bar{W}}, \\
\delta A_{\dot{\alpha}} & =\Omega_{\dot{\alpha}}+\epsilon_{\alpha \dot{\alpha}} Q \Lambda^{\alpha}+\delta_{\alpha \dot{\alpha}} \partial_{\bar{W}} \Lambda^{\alpha}+\sigma_{\alpha \dot{\alpha}}^{1} \partial_{Z} \Lambda^{\alpha}+\delta_{\alpha \dot{\alpha}} \theta^{\alpha} \Gamma_{\bar{W}}, \\
\delta B_{\alpha \beta} & =-D_{\alpha} \Omega_{\beta}, \quad \delta B_{\alpha \dot{\beta}}=-D_{\alpha} \Omega_{\dot{\beta}}+\delta_{\alpha \dot{\beta}} \Gamma_{\bar{W}}, \\
\delta B_{\dot{\alpha} \beta} & =-D_{\dot{\alpha}} \Omega_{\beta}+\delta_{\beta \dot{\alpha}} \Gamma_{\bar{W}}, \quad \delta B_{\dot{\alpha} \dot{\beta}}=-D_{\dot{\alpha}} \Omega_{\dot{\beta}}, \\
\delta C_{\alpha}^{\beta} & =-D_{\alpha} \Lambda^{\beta}-\Phi_{\alpha}^{\beta}, \quad \delta C_{\alpha}^{\dot{\beta}}=-D_{\alpha} \Lambda^{\dot{\beta}} \\
\delta C_{\dot{\alpha}}^{\beta} & =-D_{\alpha} \Lambda^{\beta}, \quad \delta C_{\dot{\alpha}}^{\delta \beta}=-D_{\dot{\alpha}} \Lambda^{\dot{\beta}}-\Phi_{\dot{\alpha}}^{\dot{\beta}}, \\
\delta H_{W \alpha} & =-\delta_{\alpha \dot{\alpha}} \Lambda^{\dot{\alpha}}+D_{\alpha} \Gamma_{W}, \quad \delta H_{W \dot{\alpha}}=-\delta_{\alpha \dot{\alpha}} \Lambda^{\alpha}+D_{\dot{\alpha}} \Gamma_{Z}, \\
\delta H_{\bar{W} \alpha} & =D_{\alpha} \Gamma_{\bar{W}}, \quad \delta H_{\bar{W} \dot{\alpha}}=D_{\dot{\alpha}} \Gamma_{\bar{W}}, \\
\delta K_{\alpha} & =-\sigma_{\alpha \dot{\alpha}}^{1} \Lambda^{\dot{\alpha}}+D_{\alpha} \Gamma_{\bar{Z}}, \quad \delta K_{\dot{\alpha}}=-\sigma_{\alpha \dot{\alpha}}^{1} \Lambda^{\alpha}+D_{\dot{\alpha}} \Gamma_{\bar{Z}}, \\
\delta F_{\alpha \gamma}^{\beta} & =D_{\alpha} \Phi_{\gamma}^{\beta}, \quad \delta F_{\dot{\alpha} \dot{\gamma}}^{\dot{\beta}}=D_{\dot{\alpha}} \Phi_{\dot{\gamma}}^{\dot{\beta}} .
\end{aligned}
$$

By using the gauge parameters $\Omega_{\alpha}, \Omega_{\dot{\alpha}}, \Gamma_{\bar{W}}, \Phi_{\alpha}^{\beta}, \Phi_{\dot{\alpha}}^{\dot{\beta}} \Lambda^{\alpha}$ and $\Lambda^{\dot{\alpha}}$ we can set

$$
A_{\alpha}=A_{\dot{\alpha}}=C_{\alpha}^{\beta}=C_{\dot{\alpha}}^{\dot{\beta}}=H_{W \alpha}=H_{W \dot{\alpha}}=H_{\bar{W} \alpha}=H_{\bar{W} \dot{\alpha}}=0,
$$

where $H_{\bar{W}}$ are set to zero after using (D.28). From the equations of motion in this gauge we get that

$$
F_{(\alpha \beta)}^{\gamma}=F_{(\dot{\alpha} \dot{\beta})}^{\dot{\gamma}}=0, \quad C_{1}^{\dot{1}}=C_{2}^{\dot{2}}=C_{1}^{\dot{2}}+C_{2}^{\dot{1}}=C_{\dot{1}}^{1}=C_{\dot{2}}^{2}=C_{\dot{1}}^{2}+C_{\dot{2}}^{1}=0 .
$$


Using the equations of motion now we can solve for

$$
B_{\alpha \dot{\beta}}=D_{\alpha} T_{\dot{\beta}}, \quad B_{\dot{\alpha} \beta}=D_{\dot{\alpha}} T_{\beta}
$$

After taking into account all the gauge invariances and the equations of motion, of the whole (D.20) we are left with the following field content. Out of the four $K_{\bar{Z}}{ }_{A}$, we keep three. We expect that these fields correspond to some higher states in the superstring cohomology. The fields sitting in the $B$ 's, that we identify with the supermultiplet the tachyon sits in, boiled down to $T_{\alpha}, T_{\dot{\alpha}}$. If we focus on these last fields, the part of the vertex operator which contains the tachyon reads $\mathcal{U}_{T}^{(1)}=$ $\lambda^{\alpha} \partial \theta^{\dot{\alpha}} D_{\alpha} T_{\dot{\alpha}}+\lambda^{\dot{\alpha}} \partial \theta^{\alpha} D_{\dot{\alpha}} T_{\alpha}$. We have to project the vertex operator to the physical supercoordinates, that in our $S O(4)$ notation are $\left(\theta^{1}, \theta^{\mathrm{i}}\right)$. Then the vertex operator reduces to

$$
\mathcal{U}_{T}^{(1)}=\lambda^{\alpha} \partial \theta^{\dot{1}} D_{\alpha} T_{\dot{1}}\left(\theta^{1}, \theta^{\dot{1}}\right)+\lambda^{\dot{\alpha}} \partial \theta^{1} D_{\dot{\alpha}} T_{1}\left(\theta^{1}, \theta^{\dot{1}}\right),
$$

where the two physical superfields $T_{1}$ and $T_{1}$ contain $2 \oplus 2$ degrees of freedom each. At this point, the structure of the pure spinor space crucially comes into play. As we discussed, the four dimensional pure spinor space $\lambda^{\alpha} \lambda^{\dot{\alpha}}=0$ is the union of two disconnected patches and our would-be vertex operator (D.44) is symmetric under the exchange of the two patches $\lambda^{\alpha} \leftrightarrow \lambda^{\dot{\alpha}}$. The RNS formalism is mapped onto just one of the two patches, let us choose the $\lambda^{\dot{\alpha}} \neq 0$ patch. If we want to compare the RNS cohomology with the pure spinor result, we are forced to do that on one of the two disconnected patches, so the tachyon supermultiplet on the patch $\lambda^{\dot{\alpha}} \neq 0$ has the form

$$
\mathcal{U}^{(1)}=\lambda^{\dot{\alpha}} \partial \theta^{1} D_{\dot{\alpha}} T_{1}\left(\theta^{1}, \theta^{\dot{1}}\right)
$$

containing $2 \oplus 2$ degrees of freedom. In the closed string, the vertex operator is just the product of the holomorphic and antiholomorphic vertex operators, which contains the tachyon supermultiplet.

\section{E. The six-dimensional map}

In this appendix we present some details about the map from the RNS to the pure spinor variables in the six dimensional case of section 6.2 in the main text. In the following discussion it will be convenient to break the $S O(6)$ group into $U(3)$ and classify the different supercharges and pure spinor components using their representation in $S U(3)_{U(1)}$, in terms of which $q_{+i}$ is in the representation $1_{\frac{3}{2}}$, while $q_{+1}, q_{+2}$ and $q_{+\dot{2}}$ form a $3_{-\frac{1}{2}}$ representation.

We will work in the patch in which the $1_{\frac{3}{2}}$ component of the pure spinor is non-zero and raise the $1_{\frac{3}{2}}$ supercharge $q_{+i}$ to picture $\frac{1}{2}$ and obtain

$$
q_{+\dot{1}}=b \eta e^{3 \phi / 2+i\left(H^{1}+H^{2}+H-Q x\right) / 2}+\ldots,
$$


where $\ldots$ are terms with lower exponentials of the field $\phi$. The $3_{-\frac{1}{2}}$ is left in the $-\frac{1}{2}$ picture. We further define the fermionic momenta

$$
\begin{aligned}
& p_{+\mathrm{i}}=b \eta e^{3 \phi / 2+i\left(H^{1}+H^{2}+H-Q x\right) / 2}, \quad p_{+1}=e^{-\phi / 2+i\left(H^{1}-H^{2}-H+Q x\right) / 2}, \\
& p_{+2}=e^{-\phi / 2+i\left(-H^{1}+H^{2}-H+Q x\right) / 2}, \quad p_{\dot{+} \dot{2}}=e^{-\phi / 2+i\left(-H^{1}-H^{2}+H+Q x\right) / 2} .
\end{aligned}
$$

The last momentum is not a physical supercharge. It is taken instead of $q_{+\dot{2}}$, which is singular with $q_{+1}$ and $q_{+2}$ because of the supersymmetry algebra. The non-physical $p_{\dot{+} \dot{2}}$ is non-singular with all the others. We also define the coordinates conjugate to these momenta

$$
\begin{aligned}
& \theta^{+\dot{1}}=c \xi e^{-3 \phi / 2-i\left(H^{1}+H^{2}+H-Q x\right) / 2}, \quad \theta^{+1}=e^{\phi / 2-i\left(H^{1}-H^{2}-H+Q x\right) / 2} \\
& \theta^{+2}=e^{\phi / 2-i\left(-H^{1}+H^{2}-H+Q x\right) / 2}, \quad \theta^{\dot{+}}=e^{\phi / 2-i\left(-H^{1}-H^{2}+H+Q x\right) / 2} .
\end{aligned}
$$

We now map

$$
\eta=e^{\tilde{\phi}+\tilde{\kappa}} p_{+\dot{1}}, \quad b=e^{(\tilde{\phi}-\tilde{\kappa}) / 2} p_{+\dot{1}}
$$

from which we obtain

$$
\begin{aligned}
& \tilde{\phi}=-\frac{i}{4}\left(3 H+3 H^{1}+3 H^{2}-3 Q x-4 i \kappa-9 i \phi+2 i \chi\right), \\
& \tilde{\kappa}=\frac{i}{4}\left(H+H^{1}+H^{2}-Q x-4 i \kappa-3 i \phi-2 i \chi\right),
\end{aligned}
$$

which are non-singular with the fermionic momenta and satisfy the OPE's

$$
\tilde{\phi}(z) \tilde{\phi}(0) \sim-\log z, \quad \tilde{\kappa}(z) \tilde{\kappa}(0) \sim \log z
$$

and all the other OPE's are non-singular.

The coordinate $x$ is singular with the new variables. This is solved by performing the shift

$$
x^{\prime}=\frac{1}{\sqrt{2}}\left(i \phi-H^{1}-H^{2}-H\right) .
$$

(A definition with the opposite signs is also possible.) It is curious that the new $x^{\prime}$ is independent of the original $x$.

Hence, the RNS bosonic fields $x, \beta$ and $\gamma$ are mapped into the pure spinor bosons $x^{\prime}, \tilde{\phi}$ and $\tilde{\kappa}$, while the bosonic coordinates $\varphi$ and $x^{\mu}(\mu=1, \ldots, 4)$ are mapped into themselves. The eight fermionic RNS variables $\psi_{l}, \psi, \psi^{\mu}, b$ and $c(\mu=1, \ldots, 4)$ are mapped to the eight fermionic coordinates and momenta. One can turn the RNS energy-momentum tensor into pure spinor fields on this patch and obtain

$$
\begin{aligned}
T^{\prime}= & -\frac{1}{2} \partial x^{\mu} \partial x^{\nu} \eta_{\mu \nu}-\frac{1}{2}(\partial \varphi)^{2}-\frac{1}{2}\left(\partial x^{\prime}\right)^{2}+\frac{Q}{2} \partial^{2}\left(\varphi-i x^{\prime}\right)- \\
& -p_{+\dot{1}} \partial \theta^{+\dot{1}}-p_{+1} \partial \theta^{+1}-p_{+2} \partial \theta^{+2}-p_{\dot{+} \dot{2}} \partial \theta^{\dot{+} \dot{2}}- \\
& -\frac{1}{2}(\partial \tilde{\phi})^{2}+\frac{1}{2}(\partial \tilde{\kappa})^{2}+\partial^{2} \tilde{\phi}+\partial^{2} \tilde{\kappa} .
\end{aligned}
$$


whose total central charge still vanishes. Next, one uses the standard bosonization of a $\beta \gamma$-system in order to relate $\tilde{\phi}$ and $\tilde{\kappa}$ to the pure spinor variables

$$
\lambda^{+\dot{1}}=e^{\tilde{\phi}+\tilde{\kappa}}, \quad w_{+\dot{1}}=\partial \tilde{\kappa} e^{-\tilde{\phi}-\tilde{\kappa}}
$$

and write the $(\tilde{\phi}, \tilde{\kappa})$ part of the energy-momentum tensor as

$$
T_{\lambda}=w_{+\dot{1}} \partial \lambda^{+i}-\frac{1}{2} \partial^{2} \log \Omega,
$$

where $\Omega$ is the top dimensional form on the pure spinor space [8, 7]. By comparison with $($ E.7) the top form is

$$
\Omega=e^{-3(\tilde{\phi}+\tilde{\kappa})}=\left(\lambda^{+\dot{1}}\right)^{-3}
$$

The above expressions are not covariant. They can be covariantized by adding the missing $3_{-\frac{1}{2}}$ and $1_{\frac{3}{2}}$ components of the weight $(1,0) b c$-systems $\left(p_{2 a}, \theta_{2}^{a}\right)$ and $\left(p_{2+}, \theta_{2}^{+}\right)$and the weight $(1,0)$ pure spinor $\beta \gamma$-systems $\left(w_{1 a}, \lambda_{1}^{a}\right)$ and $\left(w_{2+}, \lambda_{2}^{+}\right)$(we switched back to the notation introduced in the beginning of this section) allowing us to write covariantly the energy-momentum tensor (6.8). The addition of the quartet does not modify the central charge due to the cancellation between the $b c$-systems and the $\beta \gamma$-systems. The BRST operator should be modified so that these additional fields do not modify the cohomology.

\section{F. A projection}

Consider the four dimensional non-critical superstring we constructed in section 5.2 . In this appendix we propose a way to project out half of the doubled superspace. However, we do not know what target space this string theory describes.

Consider the pure spinor BRST operator we considered in the main text

$$
Q_{B}=\oint \lambda^{I i} d_{I i}
$$

and add the following contribution

$$
Q^{\prime}=\epsilon^{I 1} Q_{I 1}
$$

where $Q_{I 1}=\oint q_{I 1}$ are two of the doubled supercharges. These are defined to anticommute with the $d$ 's in (5.27), and are given by

$$
q_{I j}=p_{I j}+\frac{1}{2} \tau_{i j}\left(\delta_{I J} \theta^{J i} \partial\left(x_{1}+i x_{2}\right)+\tau_{I J} \theta^{J i} \partial(\varphi-i x)-Q \epsilon_{I J} \partial \theta^{J i}\right),
$$

such that the OPE's of the $q_{I i}$ among themselves is the same as (5.28) but with opposite signs. 
Since the supercharges and the superderivatives anticommute, the new BRST charge $\mathcal{Q}=Q_{B}+Q^{\prime}$ is nilpotent on the pure spinor constraint (5.11). Now let us look at the cohomology of this theory. Instead of computing the cohomology of (F.1) and then removing the unphysical $\theta^{+i}$ from the spectrum, as we did in the main text, we now take $\mathcal{Q}$ as the full BRST operator of the theory, without the need of a further projection. The cohomology computation for the ghost number one and weight zero vertex operators (5.37) can be computed in two steps. First, we compute the cohomology of the old $Q_{B}$, by which we obtain the "off-shell" four dimensional vector supermultiplet (5.41) with $4 \oplus 4$ states, that we can pack in a real four dimensional superfield $V$. Secondly, the action of the new term $Q^{\prime}$ is just to remove from the spectrum the dependence on half of the supercoordinates $\theta^{I 1}$. However, this is a different projection with respect to the one that realizes the linear dilaton background!

Requiring that the four dimensional vector superfield $V$ belongs to the cohomology of $\mathcal{Q}=Q_{B}+Q^{\prime}$ restricts it to

$$
\left.V\right|_{\theta^{I 1}=0}=\phi+\theta^{I 2} \psi_{I 2}+\theta^{2} F
$$

giving a total of $2 \oplus 2$ degrees of freedom as before, but with different space-time charges.

The interesting observation is that the BRST charge $\mathcal{Q}=Q_{B}+Q^{\prime}$ can be derived by a GS-like action through the usual Oda-Tonin trick [38.

Consider now the four dimensional pure spinor action in the linear dilaton background (5.22). It is invariant with respect to the pure spinor operator (E.1) but not with respect to the total BRST operator $\mathcal{Q}=Q_{B}+Q^{\prime}$. In fact, the variation of the action with respect to $Q^{\prime}$ in ( $(\underline{F .2})$ is $\left[Q^{\prime}, S\right]=\epsilon^{I 1} \int d^{2} z \bar{\partial} d_{I 1}$. By applying a simple descent method, we find that to restore the invariance of the action under the total BRST symmetry we need to add the term

$$
S_{\text {new }}=\epsilon^{I 1} \int d^{2} z \bar{\partial} w_{I 1}
$$

whose variation is $\left[Q_{B}, S_{\text {new }}\right]=-\left[Q^{\prime}, S\right]$. The total action $S+S_{\text {new }}$ is now invariant with respect to the total BRST operator. This analysis seems to produce a consistent projection. What is the resulting string theory is not clear.

One may go the other way to get the pure spinor BRST invariant action from the GS action, where we start with a GS-like action $S_{G S}$ and consider the BRST fermion $\Psi=\int d^{2} z w_{I 1} \bar{\partial} \theta^{I 1}$, following the procedure of [38, 10]. Then the total pure spinor action is given by

$$
S_{p s}=S_{G S}+\{\mathcal{Q}, \Psi\}
$$

In our case $\mathcal{Q}=Q_{B}+Q^{\prime}$. 


\section{References}

[1] D. Kutasov and N. Seiberg, Noncritical superstrings, Phys. Lett. B251 (1990) 67-72.

[2] A. M. Polyakov, The wall of the cave, Int. J. Mod. Phys. A14 (1999) 645-658, hep-th/9809057.

[3] A. M. Polyakov, Conformal fixed points of unidentified gauge theories, Mod. Phys. Lett. A19 (2004) 1649-1660, hep-th/0405106.

[4] P. A. Grassi and Y. Oz, Non-critical covariant superstrings, hep-th/0507168.

[5] N. Berkovits, Super-Poincare covariant quantization of the superstring, JHEP 04 (2000) 018, hep-th/0001035.

[6] N. Berkovits, Quantum consistency of the superstring in AdS(5) $x S^{* * 5}$ background, JHEP 03 (2005) 041, hep-th/0411170].

[7] E. Witten, Two-dimensional models with (0,2) supersymmetry: Perturbative aspects, hep-th/0504078.

[8] N. A. Nekrasov, Lectures on curved beta-gamma systems, pure spinors, and anomalies, hep-th/0511008.

[9] N. Berkovits, ICTP lectures on covariant quantization of the superstring, hep-th/0209059.

[10] M. Matone, L. Mazzucato, I. Oda, D. Sorokin, and M. Tonin, The superembedding origin of the Berkovits pure spinor covariant quantization of superstrings, Nucl.

Phys. B639 (2002) 182-202, hep-th/0206104.

[11] Y. Aisaka and Y. Kazama, Origin of pure spinor superstring, JHEP 05 (2005) 046, hep-th/0502208.

[12] A. Gaona and J. A. Garcia, Bft embedding of the green-schwarz superstring and the pure spinor formalism, JHEP 09 (2005) 083, hep-th/0507076.

[13] N. Berkovits and O. Chandia, Massive superstring vertex operator in $d=10$ superspace, JHEP 08 (2002) 040, hep-th/0204121.

[14] N. Berkovits and P. S. Howe, Ten-dimensional supergravity constraints from the pure spinor formalism for the superstring, Nucl. Phys. B635 (2002) 75-105, hep-th/0112160.

[15] N. Berkovits, Relating the RNS and pure spinor formalisms for the superstring, JHEP 08 (2001) 026, hep-th/0104247.

[16] D. Friedan, E. J. Martinec, and S. H. Shenker, Conformal invariance, supersymmetry and string theory, Nucl. Phys. B271 (1986) 93. 
[17] D. Kutasov, Some properties of (non)critical strings, hep-th/9110041.

[18] N. Itzhaki, D. Kutasov, and N. Seiberg, Non-supersymmetric deformations of non-critical superstrings, JHEP 12 (2005) 035, hep-th/0510087.

[19] S. Murthy, Notes on non-critical superstrings in various dimensions, JHEP 11 (2003) 056, hep-th/0305197.

[20] A. Giveon, D. Kutasov, and O. Pelc, Holography for non-critical superstrings, JHEP 10 (1999) 035, hep-th/9907178.

[21] K. Itoh and N. Ohta, Brst cohomology and physical states in 2-d supergravity coupled to c $i=1$ matter, Nucl. Phys. B377 (1992) 113-142, hep-th/9110013.

[22] N. Seiberg, Observations on the moduli space of two dimensional string theory, JHEP 03 (2005) 010, hep-th/0502156.

[23] H. Ita, H. Nieder, and Y. Oz, On type II strings in two dimensions, JHEP 06 (2005) 055, hep-th/0502187.

[24] N. Seiberg, Notes on quantum Liouville theory and quantum gravity, Prog. Theor. Phys. Suppl. 102 (1990) 319-349.

[25] N. Berkovits, A new description of the superstring, hep-th/9604123.

[26] P. A. Grassi and J. F. Morales Morera, Partition functions of pure spinors, hep-th/0510215.

[27] W. Siegel, Classical superstring mechanics, Nucl. Phys. B263 (1986) 93.

[28] N. Wyllard, Pure-spinor superstrings in d=2, 4, 6, JHEP 11 (2005) 009, hep-th/0509165.

[29] P. A. Grassi and N. Wyllard, Lower-dimensional pure-spinor superstrings, JHEP 12 (2005) 007, hep-th/0509140.

[30] J. Polchinski, String theory. vol. 2: Superstring theory and beyond, . Cambridge, UK: Univ. Pr. (1998) 531 p.

[31] N. Berkovits, Multiloop amplitudes and vanishing theorems using the pure spinor formalism for the superstring, JHEP 09 (2004) 047, hep-th/0406055.

[32] R. R. Metsaev and A. A. Tseytlin, Type IIB superstring action in AdS(5) $x S(5)$ background, Nucl. Phys. B533 (1998) 109-126, hep-th/9805028.

[33] P. Bouwknegt, J. G. McCarthy, and K. Pilch, Ground ring for the 2-D NSR string, Nucl. Phys. B377 (1992) 541-570, hep-th/9112036.

[34] P. A. Grassi, G. Policastro, M. Porrati, and P. Van Nieuwenhuizen, Covariant quantization of superstrings without pure spinor constraints, JHEP 10 (2002) 054, hep-th/0112162. 
[35] N. Berkovits and N. Nekrasov, The character of pure spinors, Lett. Math. Phys. 74 (2005) 75-109, hep-th/0503075.

[36] A. Connes and M. Dubois-Violette, Yang-Mills algebra, Lett. Math. Phys. 61 (2002) 149-158, math.qa/0206205.

[37] O. Aharony, A. Giveon, and D. Kutasov, Lsz in lst, Nucl. Phys. B691 (2004) 3-78, hep-th/0404016.

[38] I. Oda and M. Tonin, On the Berkovits covariant quantization of GS superstring, Phys. Lett. B520 (2001) 398-404, hep-th/0109051]. 